\title{
The effect of subgingival
} antimicrobial therapy on the

levels of stromelysin and

tissue inhibitor of

metalloproteinases in

gingival crevicular fluid

\author{
By \\ Nahid Pourtaghi \\ D.D.S (Iran)
}

Thesis submitted for the degree of Master of science to the Faculty of Medicine, University of Glasgow

Unit of Periodontology, Department of Adult Dental Care, University of Glasgow Dental School

(C) N.Pourtaghi, November 1995 
ProQuest Number: 11007786

All rights reserved

INFORMATION TO ALL USERS

The quality of this reproduction is dependent upon the quality of the copy submitted.

In the unlikely event that the author did not send a complete manuscript and there are missing pages, these will be noted. Also, if material had to be removed, a note will indicate the deletion.

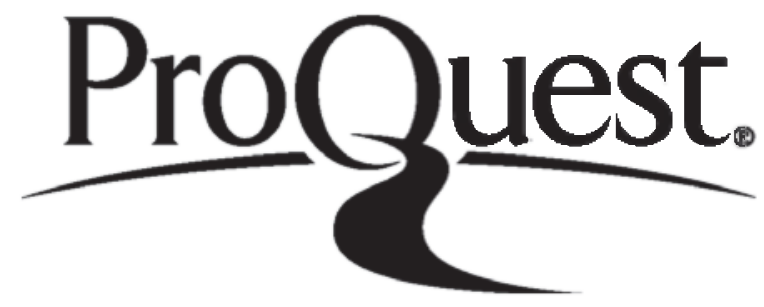

ProQuest 11007786

Published by ProQuest LLC (2018). Copyright of the Dissertation is held by the Author.

All rights reserved.

This work is protected against unauthorized copying under Title 17, United States Code Microform Edition (C) ProQuest LLC.

ProQuest LLC.

789 East Eisenhower Parkway

P.O. Box 1346

Ann Arbor, Ml $48106-1346$ 
Therir
10450
Coppl 


\section{TABLE OF CONTENT}

CONTENTS

IIST OF FIGURES

VII

LIST OF TABLES

IX

ABBREVIATIONS

XII

ACKNOWLEDGEMENTS

XV

DECLARATION

XVIII

SUMMARY

XIX

\section{CONTENTS}

CHAPTER 1: Introduction

1.1

The 'Normal' periodontium; Clinical and histological features

1.2

Periodontal disease; Clinical and histological features

1.3

Recent concepts in the epidemiology

of periodontal disease

1.4

Pathogenesis and progression

of periodontal disease

1.5

The features of destruction in

periodontitis

1.6

Origin, methods of collection and volume determination of gingival crevicular fluid and rationale for gingival crevicular fluid sampling

1.7 .1

Sources, the Matrixin family, the

structure of matrixin and the molecular biology of the matrix metalloproteinases 
1.7.3 Regulation of matrix metalloproteinases

1.7.4 Tissue inhibitors of matrix

1.7 .5 metalloproteinase (TIMP)

1.7 .6

Other inhibitors of matrixin

The role of matrix metalloproteinases in the pathogeneses of the periodontal disease

1.8

Treatment of periodontal disease

27

1.8 .1

Antibiotics in the management of periodontal disease

1.8 .2

Rational for the use of antibiotics in periodontal disease

1.8 .3

Common antibiotics used in the treatment of periodontal disease

1.8 .4

Local versus systemic delivery of antibiotics in periodontitis therapy

1.8 .5

Clinical efficacy of antibiotics in periodontal disease

1.8 .6

Additional effects of antibiotics

$1.8 \cdot 6.1$

Anti-inflammatory effects of

1.8 .6 .2

Anti-collagenase activities of tetracyclines

1.9

Aim

\section{CHAPTER 2: Materials and Methods}

2.1

Subjects

2.2

Selection of periodontal sites

2.3

Clinical design 

therapy on matrix metalloproteinase and inhibitor in gingival crevicular fluid method

2.7.1 Calibration of the periotron 6000 for use in clinical trials 
2.8 Preliminary experiments

2.8 .1

The reproducibility of stromelysin (SL)

and tissue inhibitor of

metalloproteinase (TIMP) on gingival

crevicular fluid paper strip samples

2.9

Statistical Analysis

3.1

A cross-sectional analysis of association between GCF SL and TIMP levels and clinical parameters

3.1 .1

Comparison of GCF levels of stromelysin

and TIMP between bleeding and non-bleeding sites, suppurating and non-suppurating sites and sites with different levels of gingival inflammation at the baseline

3.1 .2

Correlation between probing depth, GCF volume and GCF levels of matrix metalloproteinases and their inhibitor

3.1 .3

Comparison between GCF level of SL, TIMP, and GCF volume, considering smoking, gender, age, and anterior vs. posterior sites

3.2

The efficacy of adjunctive locally delivered antibiotics on biochemical and clinical parameters

3.2.1 Change in the clinical finding after adjunctive treatment with different locally delivered antibiotics or scaling and root planing alone or scaling and root planing alone 
The effects of smoking, sex, site, and age on change of GCF SL, TIMP, and volume

3.2 .5

Correlation between pocket depth, GCF volume and GCF levels of $\mathrm{SL}$ and its inhibitor at reassessment

Prediction of response to treatment according to the baseline level of stromelysin and tissue inhibitor of metalloproteinases

3.2 .6 .1

Prediction of response to treatment according to the baseline levels of stromelysin and comparison of BRS and WRS in their change of stromelysin levels

3.2 .6 .2

Prediction of response to treatment 96 according to the baseline level of TIMP and comparison of BRS and WRS in their change of TIMP levels

3.2.7 Prediction of response to treatment (change in the attachment levels) according to the baseline GCF levels of stromelysin and TIMP

4.1 Clinical design

The choice of biochemical parameters of metalloproteinases 
A cross sectional analysis of the association between GCF SL and TIMP levels and clinical parameter at the baseline

4.6 .2

Comparison between GCF level of SL, TIMP and volume considering smoking, gender, age, and anterior vs. posterior sites

4.6 .3

Effects of antimicrobial treatment level of GCF SL and TIMP

4.6 .4

Correlation between clinical parameters and gingival crevicular fluid biochemical components

4.6 .5

Comparison of stromelysin and tissue inhibitor of metalloproteinase (TIMP) in sites which did or did not respond to treatment

4.6 .6

Prediction of the outcome of treatment

$4 \cdot 6 \cdot 6.1$

Prediction of treatment response: Using the pocket depth and the state of bleeding on probing

4.6 .6 .2

Prediction of treatment response: Using the attachment level changes as criteria

4.7

Further study

REFERENCES

LIST OF PUBLICATION 


\section{LIST OF FIGURES}

CHAPTER 1

Figure 1.1 Domain structure of matrix

$17-18$ metalloproteinases

Figure 1.2 Pathway for activation and inhibition of collagenase and stromelysin

CHAPTER 2

Figure 2.1 Gingival crevicular fluid sampling

Figure 2.2 The periotron

Figure 2.3 Florida probe; consists of a computer

$57-58$ interface and a portable personal computer, a foot switch, pocket depth and an attachment level ('stent') handpiece

Figure 2.4 Attachment level measurement using the Florida probe and occlusal stent

Figure 2.5 Probing depth measurement with the Florida probe

Figure 2.6 Tetracycline fibre and cyanoacrylate $60-61$ adhesive (Actisite)

Figure 2.7 Minocycline gel (Dentomycin)

Figure 2.8 Metronidazole gel (Elyzol)

Figure 2.9 Application of tetracycline fibre

Figure 2.10 Application of minocycline gel

Figure 2.11 Calibration curve of the periotron 6000 at the volume range of 0.05 to $0.2 \mu l$ and 0.2 to $1 \mu l$. Each point represents the mean of three measurements 
Figure 2.13 Typical calibration curve for (a)

$69-70$ stromelysin (SL) and (b) tissue inhibitor of metalloproteinase (TIMP) 


\section{LIST OF TABLES}

CHAPTER 1

Table 1.1 Matrixins

$15-16$

CHAPTER 2

Table 2.1 Steps and reagents for stromelysin

(SL) and tissue inhibitor of metalloproteinase (TIMP) sandwich ELISAs

Table 2.2 Control tests for sandwich ELISAs.

Standard was added at a fifth concentration of the standard curve. When the addition of a reagent was omitted the equivalent volume of the respective buffer alone was added.

Table 2.3 Comparison of Mean (SEM) GCF level of SL, TIMP and volume in the first sample and second sample $(n=620)$, using Paired t-test.

\section{CHAPTER 3}

Table 3.1 Comparison of GCF SL and TIMP levels in sites with bleeding on probing (BOP) and sites without bleeding on probing at the baseline, using Mann-Whitney $U$ test for $S L$ and two sample $t$-test for TIMP $(n=206)$. Mean \& (SEM) are given.

Table 3.2 Comparison of GCF SL and TIMP levels $82-83$ in sites with suppuration and sites without suppuration at the baseline, using Mann-Whitney $U$ test for $S L$ and Two sample $t$-test for TIMP $(n=206)$.

Table 3.3 Comparison of GCF SL and TIMP levels $83-84$ for different gingival inflammation scores at baseline, using Kruskal-Wallis test for SL and ANOVA test for TIMP $(n=206)$. 
Table 3.4 Spearman rank correlation $83-84$ coefficients between pocket depth and GCF volume and biochemical parameters $(\mathrm{pg} / 30 \mathrm{~s})$ in GCF from 206 sites in 52 patients at the baseline examination.

Table 3.5 The effects of smoking, sex, site and $84-85$ age on biochemical and clinical parameters in baseline, site was used as the unit of analysis $(n=206)$.

Table 3.6 The group mean of GCF TIMP, BOP, PI, $85-86$ MGI, Vol, PD, gender, smoking, site, and age on SL and TIMP. The grouping criteria for SL was the detectability of SL by the ELISA and TIMP $\leq 370.4=0$ and TIMP $>370.4=1$.

Table 3.7 The Wilks' lambda and significance $85-86$ level of the entry action of variables in the SL and TIMP.

Table 3.8 The number and percentage of cases classified correctly and misclassified by the detectability of SL.

Table 3.9 The number and percentage of cases classified correctly and misclassified by the dichotomous grouping of TIMP.

Table 3.10 Comparison of attachment level and $88-89$ pocket depth and gingival volume between different treatment using analysis of variance (ANCOVA), with subjects as unit of analysis. Paired t-test was used to compare before vs. after treatment.

Table 3.11 GCF SL mean reduction after therapy $89-90$ in four treatment groups, with sites as the unit of analysis.

Table 3.12 GCF SL mean reduction after therapy $89-90$ in four treatment groups, with subject as the unit of analysis.

Table 3.13 Statistical analysis of changes of $90-91$ GCF level of Stromelysin between test groups $(n=206)$, with sites as the unit of analysis. 
Table 3.14 Statistical analysis of changes of $90-91$ GCF level of stromelysin between test groups $(n=52)$, with subject as the unit of analysis.

Table 3.15 Statistical analysis of changes of $91-92$ GCF level of TIMP between test groups $(n=206)$, with site as the unit of analysis.

Table 3.16 Statistical analysis of changes of $91-92$ GCF level of TIMP between test groups $(n=52)$, with subjects as the unit of analysis.

Table 3.17 The effect of smoking, sex, site and age on changes of GCF SL, TIMP and volume after treatment. Sites was used as the unit of analysis $(n=206)$. Two sample $t$-test was utilised. Mean (SEM) are given.

Table 3.18 Correlation of PD and GCF Vol with $92-93$ SL and TIMP at the reassessment visit, with the site as the unit of analysis.

Table 3.19 Comparison of GCF stromelysin (SL), GCF, TIMP, volume (Vol) at the baseline examination between BRS and WRS, using MannWhitney $U$ test for GCF SL, two sample $t$-test for GCF TIMP and volume.

Table 3.20 Comparison of changes of GCF $95-96$ stromelysin (SL), GCF TIMP, volume (Vol) between BRS and WRS, using two sample t-test.

Table 3.21 Comparison of changes of GCF stromelysin (SL) and GCF TIMP between BRS and WRS in four different treatment, using two sample t-test.

Table 3.22 Comparison of GCF stromelysin (SL), 97-98

GCF TIMP, volume (Vol) at the baseline examination between sites with attachment gain $(\triangle A L>I)$ and sites with attachment loss $(\triangle A L<0)$, using Mann-Whitney $U$ test for GCF SL, two sample t-test for GCF TIMP, and volume. 


\section{ABBREVIATIONS}

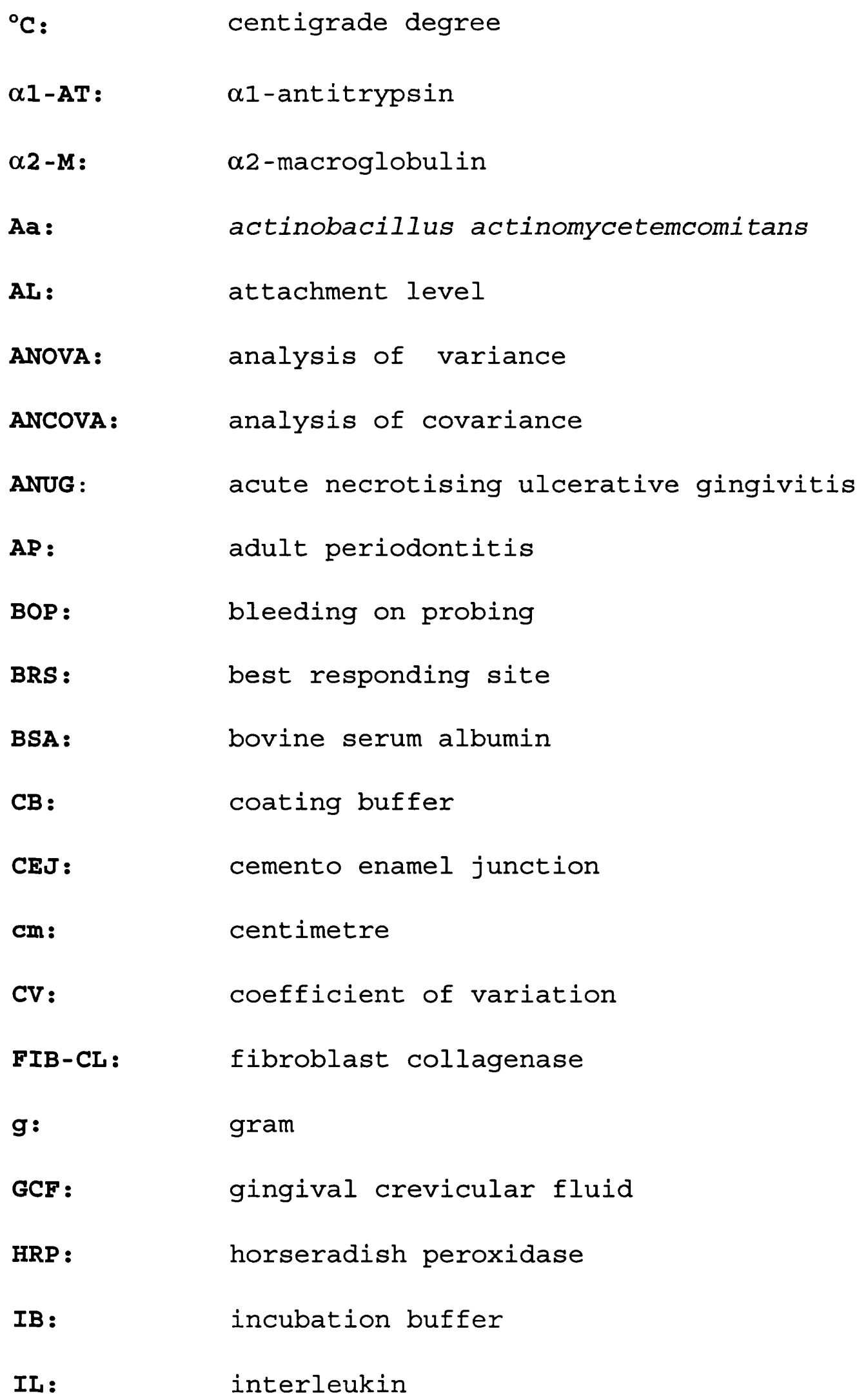




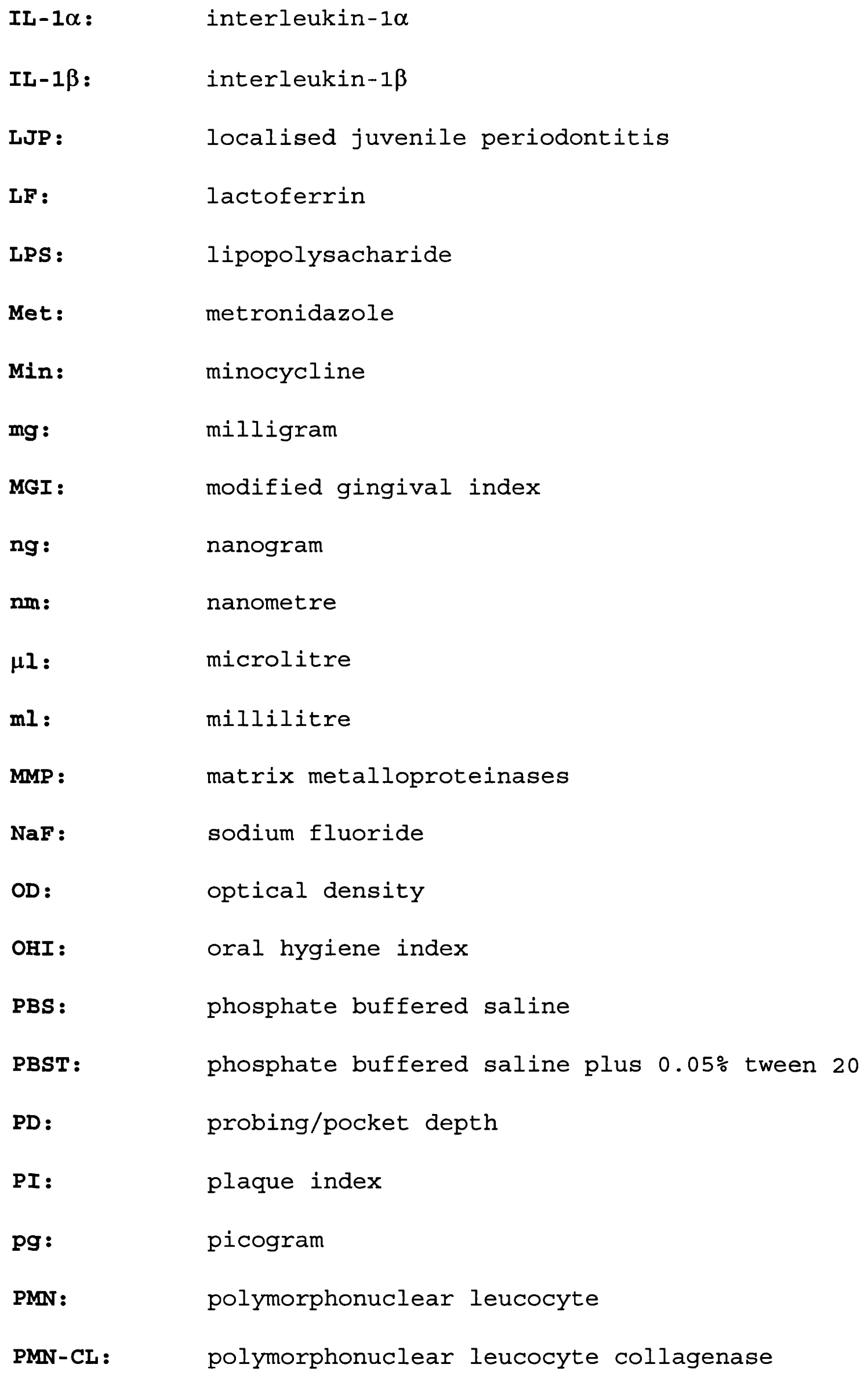




\begin{tabular}{|c|c|}
\hline RP : & refractory periodontitis \\
\hline RT : & room temperature \\
\hline SL: & stromelysin \\
\hline Su: & suppuration \\
\hline SD: & standard deviation \\
\hline SEM : & standard error of the mean \\
\hline Tc: & tetracycline \\
\hline tid: & three times a day \\
\hline TGF $-\alpha:$ & transforming growth factor $\alpha$ \\
\hline TGF-B1: & transforming growth factor $B 1$ \\
\hline TIMP : & tissue inhibitor of metalloproteinases \\
\hline TMB : & tetramethyl benzidine \\
\hline TNF- $\alpha:$ & tumour necrosis factor $\alpha$ \\
\hline vs: & versus \\
\hline WRS : & worst responding site \\
\hline WBC: & white blood cells \\
\hline
\end{tabular}




\section{ACKNOWLEDGEMENTS}

I would like to express my profound gratitude to:

My supervisor, Professor D.F. Kinane, for his support, helpful advice, encouragement and teaching throughout the postgraduate programme in Periodontics. I would like to thank him for providing me with all the clinical and laboratory facilities and equipment.

Professor K.W. Stephen, for his encouragement and for allowing me to use the facilities of the Periodontology unit of the Department of Adult Dental Care.

Iranian Ministry of Health and Medical Education for offering me the scholarship and opportunity to undertake this postgraduate study.

I would like to express my sincere thanks to the following:

All the clinical staff in the Periodontology unit of the Department of Adult Dental Care. 
All the Dental Surgery Assistants of the Periodontology unit of the Department of Adult Dental Care.

My dear colleagues and valuable friends;

Dr. M. Radvar for his support, encouragement, constructive criticism and collaboration in the study;

Dr. A. Haerian-Ardakani for his encouragement and teaching me laboratory techniques;

Mr J. Mooney for his valuable technical support;

Dr. P. Hodge, Dr. I. Chestnutt and Dr. M. Murray for critical reading of parts of this manuscript;

All the staff of the Dental Branch Library of Glasgow Dental Hospital for their valuable help;

The Department of Dental Photography of Glasgow Dental Hospital for illustration of the instruments and clinical procedures; 
All the friends and post graduate students in the Glasgow Dental School;

I would like to express my sincere gratitude and appreciation to my parents for their encouragement throughout my education.

I would like to express my profound thanks to my dear husband and my colleague, Ali for his patience during the last two years and my beloved daughter, Ania. I could not have done this study without the support of my family and it is to them I dedicate this thesis. 


\section{DECLARATION}

This thesis is the original work of the author.

Nahid Pourtaghi DDS 


\section{Summary}

Recent investigations imply that a key mechanism in the pathogenesis of periodontal disease may be the ability of oral micro-organisms to induce production and /or activation of matrix metalloproteinases in the host tissues.

Stromelysin (SL) is a member of the matrix metalloproteinase family which may play a role in chronic inflammatory periodontitis. Extracellular control of these enzymes is performed by tissue inhibitor of metalloproteinases (TIMP) and during normal tissue turnover, inflammation and healing, levels of MMPs and TIMP will change.

The effect of treatment on the levels of MMPs and inhibitors has been to decrease collagenase activity, to decrease the amount of collagenase and stromelysin (SL) produced, and to increase the level of TIMP.

It has been suggested that the pharmacologic inhibition of MMP activity could play an important role in achieving a desirable outcome in periodontal therapy. In addition to antimicrobial effects, tetracyclines have been shown to be able to inhibit metalloproteinases (MMPs). However, recent results indicate that the inhibition of MMPs by systemic tetracycline depends on the drug concentration and type as well as the origin of the MMP. Therefore, theoretically a greater inhibitory effect on MMPs would be expected if higher concentrations of drug could be delivered to the site of 
action. Recently, there has been increasing interest in the use of locally delivered antibiotics in order to obtain a high concentration of the drug at the target site and to minimise potential systemic adverse effects. We therefore sought to investigate the efficacy of locally delivered antibiotics on the level of gingival crevicular fluid (GCF) stromelysin (SL) and tissue inhibitor of metalloproteinases (TIMP) on sites with a history of a poor response to mechanical treatment.

52 patients with 4 periodontal pockets $\geq 5 \mathrm{~mm}$ and bleeding on probing were randomised into four groups of 13 patients. One group received scaling and root planing alone and the other three groups received scaling and root planing plus a locally delivered antimicrobial system. These included 25\% tetracycline fibre, 2\% minocycline gel, and 25\% metronidazole gel. The GCF samples taken at baseline and 6 weeks after treatments were analysed using an enzyme linked immunosorbent assay (ELISA).

All treatments resulted in significant improvement in clinical parameters. The pocket depth reduction was significantly greater in the scaling plus tetracycline fibre group than the scaling alone group $(p=0.003)$. GCF SL levels significantly decreased after adjunctive tetracycline fibre (paired t-test, $\mathrm{p}=0.020$ ) and minocycline gel (paired t-test, $\mathrm{p}=0.023$ ) treatments whereas it remained almost unchanged in the other two groups. While the GCF TIMP level did not change significantly in the scaling and root planing alone 
group, it significantly increased for all three adjunctive antimicrobial treatments (for tetracycline fibre p<0.001, minocycline gel $\mathrm{p}=0.005$, metronidazole gel $\mathrm{p}<0.001)$. The analysis of covariance (ANCOVA) on the baseline SL was used to reduce the error resulting from the variation in the baseline. The level of SL at baseline had a significant effect on the SL change following treatment $(p<0.001)$, and this effect was positive i.e. the higher the baseline SL, the higher the reduction of SL. Treatment also had a significant effect on the reduction of SL $(p=0.001)$. Follow-up analysis showed significant differences between; i) tetracycline fibre plus scaling and scaling alone groups $(p=0.004) ;$ and $i i)$ minocycline gel plus scaling and scaling alone groups $(p=0.002)$.

There was a significant difference in TIMP levels between treatment groups $(p=0.007)$. Furthermore, the analysis of TIMP levels revealed that a significant difference existed between the minocycline gel plus scaling and the scaling alone group $(p=0.018)$, and also between the metronidazole gel plus scaling and the scaling alone group $(p=0.001)$.

Our observation that the mean level of SL decreased after antibiotic treatment could be explained by the fact that local delivery of antibiotics plus scaling and root planing may change the microbial flora. This may cause a larger decrease of SL than scaling and root planing alone. However, the greatest decrease was observed in the sites treated by tetracycline or minocycline delivery systems. 
Previous studies have also shown that tetracyclines, in addition to decreasing the level of periodontal bacteria, resulting in decrease of MMP activity in the gingiva, could decrease the synthesis of pro-MMPs and also protect the endogenous MMP inhibitors (TIMP-1) and other proteinase imhibitors ( $\alpha 1$-antitrypsin) from degradation and inactivation by direct and indirect mechanisms. However, it cannot be established from this study whether the effect of the tetracyclines on the level of SL is direct, or indirect through the reduction in microbial challenge which could reduce the microbial proteases and inflammatory mediators such as cytokines, both of which could reduce the levels of human MMP. After treatment the level of free TIMP increased. This might be due to a reduction in MMPs which would bind to free TIMP. The elevation of GCF TIMP was higher in the metronidazole group than in the other groups. This indicates that the regulation of TIMP is not solely dependent on the MMPs. The observation of a greater clinical improvement in all three adjunctive antimicrobial treatment groups compared with the scaling and root planing alone supports our biochemical findings and suggests that the extent of healing in the patients who received local delivery antibiotics was greater than the group who only received scaling and root planing. The use of adjunctive locally delivered antimicrobial systems, particularly, the tetracycline family, may offer an advantage in changing the metalloproteinase profile of the GCF to a one more compatible with periodontal health. 
CHAPTER 1

\section{INTRODUCTION}


1.1

The 'Normal' periodontium; Clinical and histological features

The periodontium known collectively as the supporting tissues of the teeth (from the Greek, Peri, around, and odonts, tooth), are comprised of the gingivae, the periodontal ligament, the root cementum, and the alveolar bone (Lindhe, 1989).

In normal periodontium, the gingivae are pale pink in colour, scalloped in outline, have a firm consistency, does not bleed on gentle probing and terminate coronally in a knife-edge relationship with the tooth surface (Wennström, 1988). Histologically, no inflammatory exudate is found and only a few neutrophilic granulocytes can be detected within the epithelium immediately adjacent to the tooth surface (Schluger, 1990); the alveolar bone is located $1 \mathrm{~mm}$ apical to the cemento-enamel junction (Eliasson et al., 1986)

1.2

Periodontal disease; clinical and histological features

The term 'periodontal disease', if used in the general sense, includes all the pathological conditions of the periodontium. Its usage is controversial, because of the 
heterogeneity of the disease, and its multifactorial aetiology .

However, in this text, the term periodontal disease will only be used to refer to refractory disease. The clinical symptoms of periodontal disease comprise: a) redness and swelling of the gingivae; b) increased tendency to bleeding on probing the gingival sulcus/pocket; c) reduced resistance to probing; d) tissue recession; e) reduced height of the alveolar bone which is seen on the radiograph; f) mobility and drifting of the teeth which appears in the advanced stages of the disease.

1.3

Recent concepts in the epidemiology of periodontal disease

The epidemiologic approach to the study of periodontal disease was begun several decades ago. Early studies, were concerned with the discovery of the prevalence and extent of periodontal disease in populations and groups of individuals. Results of these studies, have shown that the prevalence and extent of disease increases with increasing age. The earliest study on the prevalence of periodontitis in humans was performed by Black in 1918. It was reported that loss of teeth from periodontal disease began at approximately 35 years of age, and the rate climbed 
geometrically thereafter. Hughes et al.

demonstrated that the proportions of white women and men with periodontitis were $10.8 \%$ and $13.5 \%$ respectively whereas the values for non-white women and men were $22.3 \%$ and $32.4 \%$. Hughes et al. therefore concluded, that the prevalence and severity of periodontitis increased with advancing age, were greater for non-whites than for whites, and were greater in man of both races than in women.

Schenkein et al. (1993) failed to show significant differences between the composition of the subgingival flora in males and females. The results of a 5 year clinical trial of 1,426 subjects, which was conducted to evaluate the relationship of the periodontal microflora, demographic finding, and clinical data to periodontal disease (Grossi et al., 1994) demonstrated that the prevalence of the target periodontal pathogens was greater in males than in females. Furthermore, the AfricanAmericans were more often infected with target species than white. However, the prevalence of target periodontal pathogens based on species was different in different age groups. This study also showed that subjects in younger age categories were at lower risk of clinical attachment loss. They also showed that the risk of attachment loss was greater in males compared to females. Abdellatif \& Burt (1987) suggested that while the prevalence of periodontitis may increase with age, age alone in a healthy 
adult does not lead to progression of periodontal disease. Therefore, it can also be concluded that age may correlate rather than be a risk factor for periodontal destruction. Moreover, in smokers, depending on whether the subjects were judged to be light, moderate, or heavy smokers, there is a greater risk of attachment loss in a dose dependent manner. It has also been shown that the risk of attachment loss increased in molars than premolars or anteriors (Loos et al., 1989; Nordland et al., 1987). In addition, Brayer et al. (1989) and Fleischer et al. (1989) reported that molars become calculus-free less often than single-rooted teeth. Thus, the poor response to the treatment in molars can be due to difficulty in removal of calculus in molars than other teeth.

1.4

Pathogenesis and progression of periodontal disease

Periodontal diseases are now recognised as being mixed bacterial infections of the supporting tissues of the teeth (Socransky \& Haffajee, 1991). A variety of pathogenic mechanisms and virulence factors are involved in the periodontopathic potential of organisms implicated in periodontal disease. However, Socransky \& Haffajee (1991) reported that various forms of periodontal diseases are closely associated with specific subgingival bacteria. As 
early as 1907, Goadby presumed that bacteria invade oral tissue. Several years later, Ray and Orban (1948) hypothesised that the necrosis of tissue in gingival biopsy samples was caused by bacterial invasion. During the 1960s and 1970s, again, investigators identified different bacteria and attempted to relate them to different periodontal diseases. Saglie \& Elbaz (1983) found that bacterial invasion of soft periodontal tissue and bone was common in advanced periodontitis and localised juvenile periodontitis. They also reported that bacteria invade the epithelial wall and are formed, in the enlarged intracellular spaces of the pocket epithelial surface, on the epithelial side of the basal lamina, and in the connective tissue. In subsequent studies, it was found that the organised accumulation of micro-organisms at the tooth surface triggers humoral and cellular mechanisms of host defence (Williams, 1990). Microbial release of chemotactic factors, endotoxins, cell wall fragments, toxins, and other products activate leukocyte populations that emigrate from the vasculature, and attempt to eradicate the offending pathogens (Williams, 1990). While these events are characteristic of the host response to injury, bacteria, or antigen deposition, the inability of the host to eliminate or sequester persistently accumulating numbers of pathogens that occur in the oral cavity results in prolonged inflammation. This progression to chronic periodontal disease may be associated with the 
destruction of soft tissue and alveolar bone (Williams, 1990). PMNs play a key role in the immune response and form the first line of defence in the gingival sulcus against periodontal bacteria. Quantitative (neutropenia) and qualitative (chemotactic or phagocytic) PMN deficiencies have been shown to have an important role in severe periodontal destruction (Wilton et al., 1988; Kinane \& Davies, 1990). Understanding the sequence of events of the progression to pathologic tissue injury is important in the identification of targets for modulating chronic inflammatory processes as an adjunct to antimicrobial therapy.

Three transient models have been proposed to describe the progression of periodontitis (Socransky et al., 1984). The continuous model suggested that the destruction of tooth supporting tissue proceeds in long periods of slow and constant activity with few periods of quiescence. The random or episodic burst model proposes a number of relatively short periods in which severe destruction is followed by remission and quiescence. The multiple asynchronous burst model postulates that the majority of periodontal destruction occurs during a defined period of life, and then disease activity remains essentially quiescent thereafter. 
The development of the host response and histopathogenesis were described in periodontal disease by Page and Schroeder (1976). The first stage, or the initial lesion, occurs 4 to 7 days following the accumulation of plaque. There is a classical vasculitis of the vessels subjacent to the junctional epithelium, a dramatic increase in the number of neutrophils present in the junctional epithelium in the gingival crevice, exudation of fluid from the gingival sulcus, the presence of serum proteins in the GCF, alteration in structure of the most coronal portion of the junctional epithelium, and loss of perivascular collagen. If the plaque is left undisturbed, within 4 to 7 days the early stage develops. At this stage, there is a large portion of collagen lost from the junctional epithelium, proliferation of the basal cells of the junctional epithelium begin, cytopathic effects on the gingival fibroblasts occur. There is a dramatic increase in the number of leukocytes in the junctional epithelium and gingival sulcus and the accumulation of lymphocytes in the connective tissue, which are predominantly $\mathrm{T}$ cells (cellular immune response). These two lesions characterise the acute stage of gingival inflammation. Two to three weeks of plaque accumulation are required before the third stage, referred to as the established lesion, occurs. At this stage, there is a persistence of a large 
number of plasma cells in the infiltrate, however there is no appreciable bone loss. This stage is a $\mathrm{B}$ cell response. There is greater proliferation and apical migration of the junctional epithelium, and it's lateral extension becomes more pronounced. Early pocket formation may be present. This lesion represents chronic gingivitis rather than acute gingivitis. The three previous stages define gingivitis. The fourth stage, or the advanced lesion, is characterised by loss of connective tissue attachment in root surface, and apical migration of junctional epithelium. In addition there is persistence of the histological features described for the established lesion, there is pocket formation that is associated with the loss of alveolar bone and a predominance of B cells or plasma cells. This lesion is equivalent to periodontitis.

Chronic inflammation plays an important role in periodontal destruction. It is now established that initiation and maintenance of the inflammatory process is due to factors produced/released by subgingival microbiota. For a number of years, two general mechanisms of pathogenesis have been suggested. The first involves invasion by subgingival species. It has been shown that bacterial hydrolytic enzymes can attack critical components of the intercellular matrix of epithelial and connective tissue. Recent studies indicate that some micro-organism release a variety of soluble factors which have detrimental effects on the host, such as Actinobacillus actinomycetemcomitans which produces 
leucotoxin. Apart from direct damage, other microbial substances may causes indirect damage to the host tissues. They activate endogenous cellular and hormonal inflammatory systems which affect the integrity of the periodontium. The activated cells include macrophages, lymphocytes, fibroblasts, and keratinocytes. Endothelial cells and PMNs in turn produce or release mediators such as interleukin-1 (IL-1) and tumour necrosis factor- $\alpha$ (TNF- $\alpha)$. Schwartz, Stinson \& Parker (1972) have shown that lipopolysacharides (LPS) of Gram negative bacteria interact with and modulate the behaviour of leukocytes and exhibit a wide variety of toxic and immunostimulating effects on the host. The initiation of the inflammatory reaction is due to endotoxin and bacterial derived substances which induce cytokine release from host cells, resulting in the activation of one or more of the following degradative extracellular pathways (Birkedal-Hansen et al., 1993).

The plasminogen-dependent pathway which plays an important role in the remodelling of the extracellular matrix in cell migration, trophoblast and tumour cell invasion and metastasis, and embryonic development and growth (BirkedalHansen et al., 1993).

The PMN serine proteinase pathway assumes PMN leukocytes release two serine proteinases, neutrophil elastase and cathepsin G, when triggered. These enzymes have been shown 
to have activity against several extracellular matrix proteins, however they do not cleave interstitial collagen (Birkedal-Hansen et al., 1993).

The phagocytic pathway which supposes that fibroblasts, macrophages and epithelial cells, under certain circumstances, phagocytase fragments of collagen fibrils (Birkedal-Hansen et al., 1993).

The MMP pathway which plays a substantial part in extracellular matrix destruction and remodelling in both physiological and pathological conditions (Birkedal-Hansen et al., 1993).

The osteoclastic pathway which assumes that osteoclasts initiate the resorptive process by dissolution of the layer of osteoid using a collagenase dependent proteolytic process which leads to the exposure of the underlying mineralised bone surface. The enzymatic mechanisms of organic matrix removal from bone and teeth is not completely understood (Birkedal-Hansen et al., 1993).

Apart from the above pathways, autoantibodies originating from antibodies have a role in the elimination of dead cells and constituents of damaged tissue which appear during the disease process (Anusaksathien \& Dolby, 1991). They also play a part in autoimmunity to collagen 
components (Hirsch, 1988) and depression of the host immune response during the initial stages of exposure to dental plaque (Shenker, 1987) which have been suggested to contribute to the pathogenesis of periodontal disease.

1.6

\author{
Origin, methods of collection and \\ volume determination of gingival \\ crevicular fluid and rationale for \\ gingival crevicular fluid sampling
}

Gingival crevicular fluid is an inflammatory exudate or a pre-inflammatory transudate from the gingival microcirculation present in the gingival crevice which contains several components from different sources.

The mechanisms of formation and composition of gingival crevicular fluid (GCF), and its flow into and from gingival sulci, and gingival and periodontal pockets, are not understood.

However, it has been suggested that components of the GCF may be assumed to originate from 4 main sources; a) breakdown of the host epithelial and connective tissues; b) products of host cells in the periodontium; c) plasma derived molecules; d) products derived from the subgingival microbial plaque. 
Löe and Holm-Pedersen (1965) demonstrated that the gingival fluid is an inflammatory exudate and that the flow starts before structural changes can be ascertained at the clinical level and persists some time after clinical inflammation has resolved. They also showed that the level of GCF increased with increasing gingival inflammation. Furthermore, Curtis et al. (1988) suggested that the high protein values reflect either the method of sample collection or the degree of gingival inflammation, rather than being an indicator of destructive disease in the periodontium.

Recently, Griffiths et al. (1992) investigated the association between clinical periodontal indices and both the volume and flow rate of GCF in adolescents. These investigators concluded that flow rate indicates variations in tissue permeability more precisely than clinical assessments of inflammation.

Based on the amount of fluid required and the type of analysis to be performed, methods of collection of GCF differ. Paper strip/paper points, capillary tubes or micropipettes and gingival washing are various methods of collection of GCF. Most investigators use paper strips or small micropipettes to collect GCF. When cell types and numbers in the fluid are required, gingival crevicular washings are used (Adonogianaki et al., 1993). Whereas if 
large volumes of GCF are required, capillary tubes (Larivèe, Sodek \& Ferrier, 1986) or micropipettes (Eisenhauer et al., 1983) are employed.

Gingival crevicular fluid is currently used in diagnostic tests, in the hope of: a) detecting the presence of disease and identifying patients at risk before biological onset occurs; b) evaluating the severity of disease; c) predicting the subsequent clinical course and prognosis of the disease; d) reducing the time between biological onset and usual clinical diagnosis; e) estimating the response to treatment before additional therapy is investigated; f) assessing the actual response, after completion of treatment.

The accurate and reproducible sampling of fluid is important; a number of studies have demonstrated that the choice of collection device (Brill, 1962; Sueda et al., 1969; Takamori, 1963; Oppenheim, 1970; Egelberg \& Attstrom, 1973), the various materials for sampling (Nakashima et al., 1994), the length of collection time, the number of sample repetitions (Binder et al., 1987 \& Persson \& Page, 1990) and the calculation of data as absolute measures or as flow rates (Lamster et al., 1985), contribute in a large measure to overall variability. As well as these variables, expected fluctuations based on the operatorsensitive use of collection devices exist (e.g., the depth 
of insertion of the filter-paper strip or capillary tube). The potential depletion of the sample by a prolonged collection time (Persson \& Page, 1990), the potential contamination by serum components (Lamester et al., 1985), the loss of the sample from the collection device (Rossomando, Kennedy \& Hadjimichael, 1990), and the differences between sites (Smith \& Geegan, 1991) are also factors which affect variability.

Reports have varied relating to a positive correlation between the quantity of GCF and clinical signs of gingival inflammation. The rate of renewal of epithelial structures, and the passage of leukocytes through the junctional epithelium modulate the concentration of various enzymes in the gingival crevice. This concentration will also depend upon the endogenous production of enzymes, either in the periodontal tissues or transported locally by blood. Furthermore, the presence of bacteria significantly modifies the enzymatic content of the crevice (Cimasoni, Ishikawa \& Jaccard, 1977).

The matrix matalloproteinases (MMPs) are a group of zinc enzymes which are responsible for remodelling and degradation of the extracellular matrix components. 

of matrixin and the molecular biology of the matrix metalloproteinases

The MMPs comprise at least nine members, each of which is the product of a different gene (Woessner, 1991; Birkedal et al., 1993; Murphy \& Docherty, 1992) (Table 1.1). Metalloproteinases are classified, based on substrate characteristics into three subclasses: collagenases (MMP-1) and (MMP-8), gelatinases (MMP-2 and MMP-9), and stromelysins (MMP-3, MMP-10 and MMP-11).

Collagenases are produced from different sources such as polymorphonuclear neutrophils and resident fibroblasts and also from other cells e.g. keratinocytes, endothelial cells, monocytes/macrophages, osteoblasts, and chondrocytes. Two types of mammalian collagenases have been identified in the GCF, the fibroblast type (matrix metalloproteinase 1/MMP-1) and neutrophil collagenase (MMP8). These enzymes are different gene products, they principally catalyse the same reaction, but differ in their antigenic properties (Hasty, et al., 1984), and molecular size (Sorsa et al., 1987, 1988). The enzyme of fibroblast origin has a smaller molecular mass than the one of PMN origin. Metalloproteinases which are produced by fibroblasts are synthesised by initial transcription of the corresponding genes which lasts for a period of 6 to 12 
Table 1.1 Matrixin

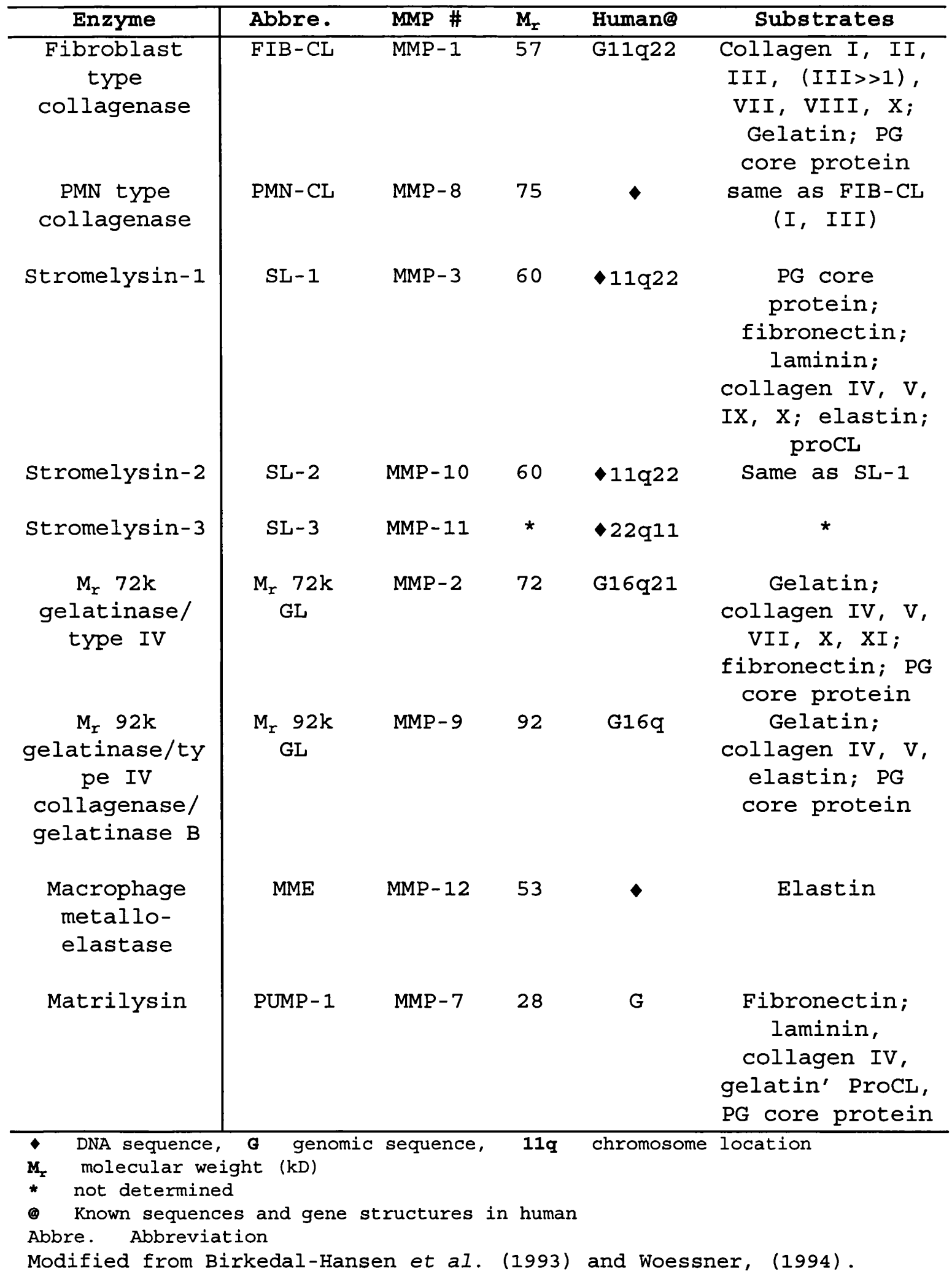


hours before the enzyme can be detected in the extracellular environment. Once the enzyme can be detected production and secretion can be sustained for several days. The PMN-collagenases are synthesised during the development of PMNs and stored in specific granules and are rapidly released when the cell is triggered.

The stromelysins include SL-1, SL-2, and SL-3 which cleave a wide rang of extracellular matrix proteins such as proteoglycan core protein, fibronectin, laminin, and type IV and $\mathrm{V}$ collagen. They have identical substrate specificities. The enzymes do not appear to be expressed by PMN leukocytes and keratinocytes in the human, but SL-1 is expressed by stromal cells either constitutively or after induction by growth factors/cytokines such as IL-1 and $\mathrm{TNF}-\alpha$.

Gelatinase is produced by keratinocytes, PMN leukocytes, and monocytes/macrophages, and occasionally by fibroblasts. GL has been identified in fibroblasts, keratinocytes, endothelial cells, monocytes/macrophages, osteoblasts, and chondrocytes. Gelatinases cleave gelatin and also types IV, V, VII, and $\mathrm{X}$ collagens and elastin. They are expressed by PMN leukocytes, Keratinocytes, monocytes and fibroblasts and also are present in a circulating form in the plasma (reviewed by Birkedal-Hansen et al., 1993). 
The matrixins have several structural features in common that include a) a propeptide domain that contains the 'cysteine switch"'; b) a catalytic zinc-binding domain; and c) a hemopexin-like domain (Birkedal-Hansen et al., 1993; Docherty \& Murphy, 1990). The propeptide domain (Nterminal) that contains the 'cysteine switch', is not required for catalysis but does play a role during the cell membrane-mediated activation of the proenzyme (Murphy et al., 1992). Matrix metalloproteinases are secreted as a proenzyme and then activated by a proteolytic process which removes an amino acid propeptide from the $\mathrm{N}$-terminus. The catalytic domain (c-terminal domain) includes the zincbinding domain that acts as a catalytic base and contains the active site of the enzyme. The c-terminal domain modulates the binding of TIMPs to the active centres of the matrixins and maintains latency of the matrixins until a signal for activation is given (Woessner, 1994). The function of the hemopexin/vitronectin domain is not completely clear. TIMPs bind to the hemopexin domain of the enzyme, because it is close enough to the active centre to interfere with the autoactivation step and with the activated form of the enzyme (Figure 1.2).

Woessner (1994) outlined the four criteria required for membership of the family matrixins of MMPs: a) the enzyme should display proteolytic activity; b) the enzyme should function outside the cell; c) its cDNA should code for a 

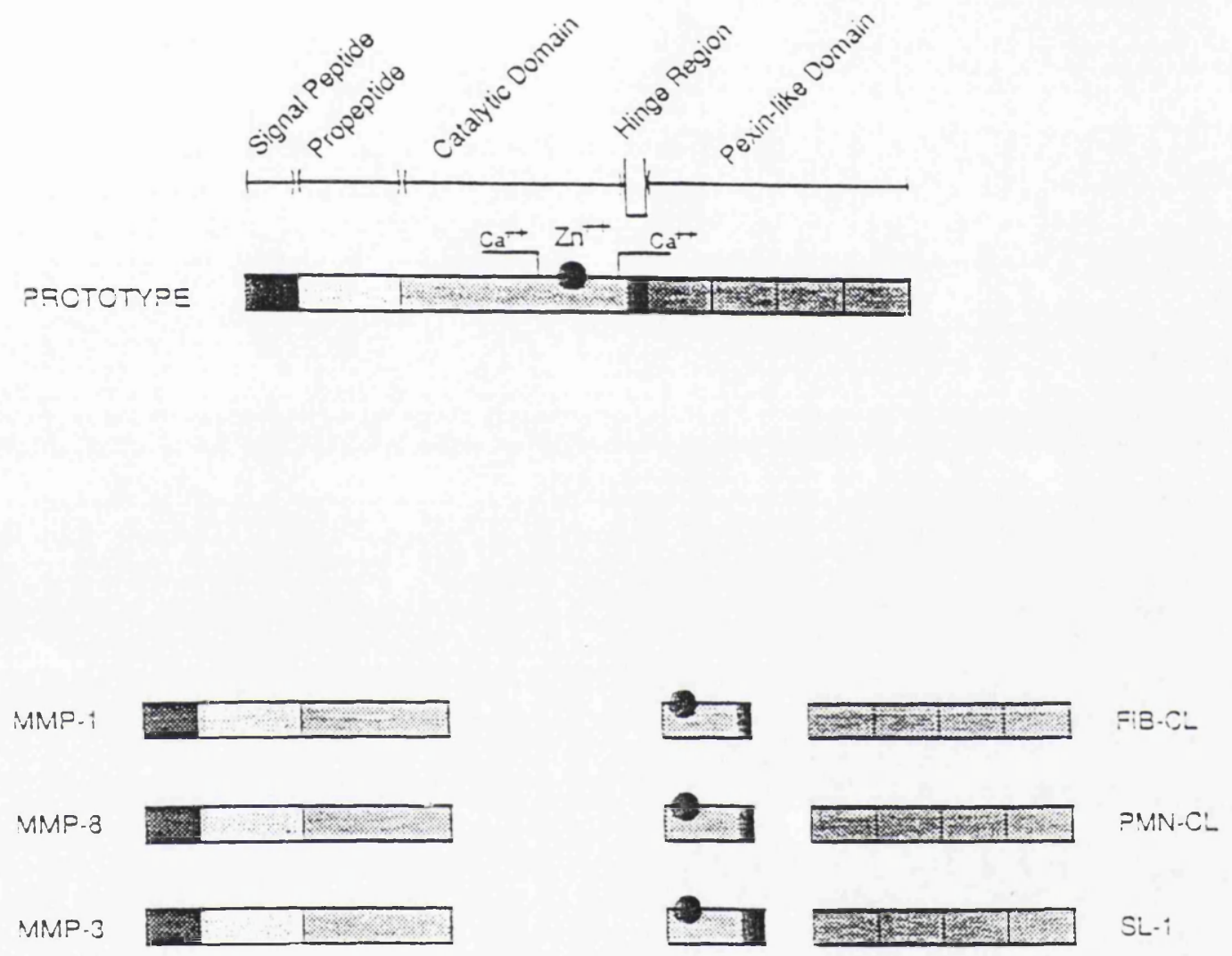

Figure 1.I Domain structure of matrix metalloproteinases Modified from Birkedal-Hansen et al. (1993). 
protein sequence for the cysteine switch mechanism; d) its CDNA should code for a protein sequence for the binding of catalytic zinc.

\section{7 .2}

Activation of matrix metalloproteinases

The MMPs are synthesised in a proenzyme form, and activation occurs after leaving the cell. In vivo, it is likely that the procollagenases are activated by proteolytic cleavage catalysed by other proteinases such as plasmin. Plasminogen may be expected to enter from the blood vessels and the mesenchymal cells can produce plasminogen activators; the activity of stromelysin is probably required for complete activation (Figure 1.2) (Harris \& Vater, 1982; Goldberg et al., 1990). Upon activation, the propeptide domain is cleaved away in several steps. Metalloproteinases can be activated by so many different agents in vitro, such as organomercurials, trypsin and plasmin. The activation by plasmin is still under question; there may be other activating proteinases. Furthermore, it has been shown that P.gingivalis can activate latent FIB-CL and to a lesser extent PMN-CL (Sorsa et al., 1992). 


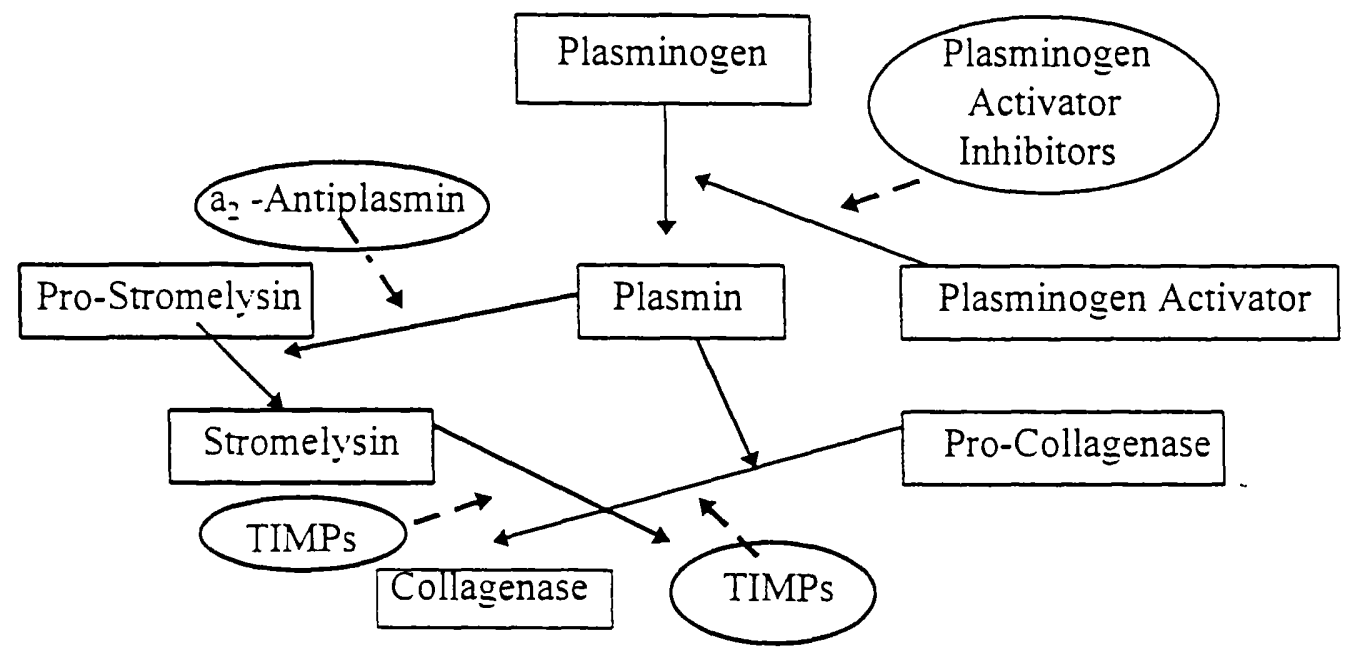

Figure 1.2 Pathways for activation and inhibition of collagenase and stromelysin

Modified from Krane, 1994. 
The matrixins are regulated at many points; a) Regulation occurs at the level of gene expression; b) Regulation at the level of secretion is recently been shown to be important in epithelial cells; c) Regulation at the level of zymogen activation has been performed by inhibitors of the extracellular matrix. Collagenase, stromelysin-1, and TIMP's regulation has co-ordinated by a variety of stimuli (Brinckerhoff et al., 1979; Werb \& Reynolds, 1974; Wrana et al., 1986). Although, early workers believed that 'coordinated regulation,' was a general feature of MMP and TIMP regulation, it is now clear that MMP and TIMP expression can be independently regulated (Overall, 1994).

Briefly, extracellular regulation of MMP activity can be achieved in four different ways namely: a) Transcriptional regulation of MMP genes; b) Precursor activation; c) differences in substrate specificity; and d) MMP inhibitors.

\section{Transcriptional regulation}

Cytokinases, morphogens and growth factors involved in cellular differentiation and tissue morphogenesis such as transforming growth factor- 11 (TGF-BI) (Overall, 1994), have been shown to regulate the expression of inhibitors of 
matrix metalloproteinases (TIMPs). TGF- $\beta$ I decreased the production of collagenase and amplified the induction of TIMP-1, mediated by epidermal growth factor and fibroblast growth factor (Edwards et al., 1987). In contrast, other cytokines, such as interleukin-1 (IL-1) (Murphy et al., 1985), epidermal growth factor and fibroblast growth factor, and tumour necrosis factor- $\alpha$ (TNF- $\alpha$ ) (Meikel et al., 1989) increase collagenase, stromelysin-1, and TIMP-1 expression. Whereas concanavalin A induces MMP production and decreases TIMP-1 and TIMP-2 synthesis (Overall et al., 1991).

\section{Activation of precursor}

The MMPs are synthesised in an inactive form that is later activated. It is likely that the procollagenases are activated by proteolytic cleavage catalysed by other proteinases such as plasmin. The activity of stromelysin is probably required for complete activation (Goldberg et al., 1990; Woessner, 1991). Therefore, the process of activation of the precursors in the extracellular matrix, is another stage in the regulation of MMP.

\section{Substrate Specificity}

The different types of substrate have been shown to be one of the factors involved in regulation of MMP activity. 
Accordingly, regulation of cell function is affected by changes in the extracellular matrix composition. At the tissue level, this affects tissue morphology and function, while at the cellular level, changes in the composition of the extracellular matrix influence cell adhesion, migration, differentiation, and activity. The enzymes have some overlapping effects on the substrate, however there are some differences with respect to their function. Interstitial collagen fibrils are dissolved by FIB-CL and PMN-type collagenases, however this ability is not shared by other members of the family.

\section{MMP Inhibitors}

Regulation at the sites of zymogen activation can be performed by inhibitors of the extracellular matrix. These are chiefly $\alpha 2$-macroglobulin and members of the TIMP family. $\quad \alpha 2$-macroglobulin plays an important role in the regulation of MMP activity by rapid capture of the proteinase. Tissue inhibitors of metalloproteinases (TIMPs) bind to the active forms of the enzyme forming $1: 1$ complexes and block their activity.

Overall (1994) demonstrated that MMPs can be independently regulated and suggested that MMP and TIMP levels in tissue extracts or inflammatory exudates do not necessarily 
reflect the response of individual cells, but rather of the cell population as a whole.

1.7 .4

\author{
Tissue inhibitors of matrix \\ metalloproteinase (TIMP)
}

TIMPs are glycoproteins and form firmly bound complexes with metalloproteinases resulting in the blocking of their activity (Cawston et al., 1983). Three forms of TIMP have been identified, TIMP-1 and TIMP-2 are secreted by many cells (Stetler-stevenson, Krutzsch \& Liotta, 1989; Welgus \& Stricklin, 1983) and are found in serum, amniotic fluid (Welgus \& Stricklin, 1983; Murphy, Cawston \& Reynolds, 1981), and saliva (Drouin et al., 1988). Their regulation at the gene level appears to be very different, TIMP-1 expression is very tightly controlled by many cytokines and growth factors whereas little has yet been found that regulates TIMP-2. The two TIMP genes are differently regulated. TIMP-3 is less well understood. All three types of TIMP consist of two structurally distinct domains; the $\mathrm{N}$-domain which reacts with the active centres of most of the matrixins, and the C-terminal domain, a functional MMP inhibitor, which interacts with the enzyme's catalytic domain. All TIMPs form high-affinity, noncovalent, and reversible complexes with the active forms of MMPs. TIMPs mechanism of inhibition is unknown. TIMPs bind to MMPs, 
this binding is in the hemopexin domain, but it is close enough to the active centre to interfere with the autoactivation step and with the activated form of the enzymes. TIMP is also known to inhibit leukocyte collagenase less effectively than the fibroblast type interstitial collagenases (Bunning et al., 1984). Activated forms of FIB-CL, as well as SL-1 and SL-2, form complexes with both TIMP-1 and TIMP-2 whereas the fulllength latent proenzymes do not (Bodden et al., 1994). Thus, remodelling of connective tissue matrices can be controlled through the precise modulation of MMP and TIMP expression.

1.7 .5

other inhibitors of matrixin

The extracellular protease activity is modulated by broad spectrum inhibitors, mainly $\alpha 1$-antitrypsin $(\alpha 1-A T)$ and $\alpha 2$-macroglobulin $(\alpha 2-\mathrm{M})$ which accounts for more than $95 \%$ of metalloproteinase inhibition in plasma. Both $\alpha 1-$ antitrypsin and $\alpha 2$-macroglobulin are detected in gingival crevicular fluid during periodontal disease (Adonogianaki et al., 1992; Gustafsson et al., 1992). The evidence suggests that $\alpha 2$-macroglobulin plays an important role in the regulation of MMP activity. The rapid capture rates, particularly with collagenase, render the reaction all but instantaneous. Cawston and Mercer (1986) showed that in a 
mixture of TIMP and $\alpha 2-M$, collagenase binds preferentially to $\alpha 2-M$. It is also of note that the concentration of $\alpha 2-M$ in the interstitial fluid is quite considerable. $\alpha 2-\mathrm{M}$ concentrations in gingival crevicular fluids (GCFs) from variously inflamed sites have yielded values from 0.2 to $2.4 \mathrm{mg} / \mathrm{ml}$. These studies suggest that $\alpha 2-\mathrm{M}$ concentrations in interstitial fluids are as much as 100 to 1000 fold greater than those of TIMPs.

The protease inhibitor complex is rapidly eliminated by the reticuloendothelial system, from the circulation by the liver and from local areas of inflammation by phagocytes (Travis \& Salvesen, 1983).

$1.7 \cdot 6$

The role of matrix metalloproteinases in the pathogeneses of the periodontal disease

Numerous studies have shown MMPs as key players in periodontal tissue destruction (Sorsa et al., 1988; Kinane, 1992; Ingman et al., 1994b, 1995). The detection of pathologically elevated levels of MMPs in extracts of inflamed gingival tissues, GCF, plaque, and salivary/mouthrinse samples has been recorded. It has been found that collagenase activity (Villela et al., 1987), and also the amount of these enzymes in GCF are increased with increasing severity of periodontal disease (Haerian et al., 
1995a). Kryshtalskyj, Sodek \& Ferrier (1986), Kryshtalskyj \& Sodek (1987) and Larvièe et al., (1986) demonstrated that the activity of these proteinases was positively correlated with severity of periodontal inflammation and pocket depth of diseased sites. However, Uitto, Suomalainen and Sorsa, (1990) demonstrated that in saliva obtained from periodontitis patients, collagenase was recovered in an active rather than in a latent form whereas in healthy individuals collagenase was partially latent. The origin of these enzyme in saliva was PMN. Furthermore, it has been shown that periodontal therapy decreases collagenase activity (Larivèe et al., 1986; Hakkarainen, Uitto \& Ainamo, 1988; Yanagimura, Koike \& Hara, 1989) and also decreases the amount of collagenase and stromelysin (SL) in the GCF (Haerian et al., 1995b). Sorsa et al. (1995) reported that in dental plaque from adult periodontitis, human and bacterial collagenase activity can be distinguished in both supra and sub gingival dental plaque.

Data from previous research indicates that a combination of debridement and antibiotic administration may reduce the incidence of active lesions in patients who appear to be refractory to conventional treatment (Loesche et al., 1984; Gusberti, Syed \& Lang, 1988). However, the cellular sources of MMPs present in inflamed human gingiva, GCF, dental plaque, and saliva and also the type of collagenases found in different categories of periodontitis are important to 
the understanding of the pathogenic mechanisms of periodontal disease. Therapeutic strategies may be directed toward reducing pathologically excessive MMP levels in different forms of periodontal disease (Golub, Suomalainen \& Sorsa, 1992). Recently, Sorsa et al. (1994) investigated the presence of different molecular forms of tissue inhibitors of matrix metalloproteinases (TIMP1 and TIMP2) in dental plaque, GCF and saliva, by Western blotting, using specific antisera. These workers discovered that latent pro MMP-8 and active MMP-8 were found in the saliva and GCF of individuals with periodontal disease. Whereas they were not found in GCF of healthy subjects. MMP-8 was found in sites with high levels of inflammation in localised juvenile periodontitis (LJP) subjects. TIMP-1 and TIMP-2 were found in the dental plaque and saliva of patients with adult periodontitis and LJP, whereas these enzymes were not detected in the GCF of these groups of patients.

Accordingly, based on the above findings and those of other studies, the authors concluded that the following factors have an effect on the results: a) the form of periodontitis which the subject has; b) the type and origin of the proteins being analysed; c) the laboratory techniques employed; and d) the way in which the results are expressed. 
The purposes of treatment in periodontal disease are as follows: a) to control or eliminate microbial plaque; b) to arrest the progression of further destruction; c) to restore function and aesthetics; d) to prevent the recurrence of the disease. These aims are accomplished by; a) motivating and teaching the patient to perform oral hygiene effectively; b) scaling and root planing; c) the removal of additional retention factors for plaque such as, overhanging margins or restorations (Lindhe, 1989).

Mechanical instrumentation produces not only a reduction in the microbial mass within subgingival pockets but also causes an alteration in the subgingival ecosystem and hence reduces the proportion of periodontopathic micro-organisms returning the flora to one more compatible with health (Magnusson et al., 1984).

The effect of mechanical debridement compared with the use of an antibiotic is superior in terms of reducing the total amount of plaque micro-organisms, nevertheless antibiotic therapy can be effective in the direct elimination of specific pathogens. 


\section{disease}

Antibiotics are thought to reduce the pathogenicity of the microflora, and hence affect the clinical signs of periodontal disease (Reviewed by Symour \& Heasman, 1995). Antibiotics have often been used against acute lesions elsewhere in the body, and it has been difficult to justify a similar efficacy in the treatment of periodontal disease. At present, there seems to be no overall agreement as to the use of antibiotics in the treatment of periodontitis, although many periodontists appear to accept their prescription for controlling some forms of rapidly progressive and refractory disease (Slots \& Rams, 1990; Fiehn, 1990; Van Winkelhoff \& de Graaff, 1991; McCulloch et al., 1990). The following section will discuss the antibiotics which are used in periodontal disease.

1. 8.2

Rational for the use of antibiotics in periodontal disease

In recent years, it has been found that micro-organisms are directly involved both in the initiation and the progression of destructive periodontal disease. The evidence to support this contention has been extensively documented in a number of reviews (Genco, Evans \& Ellison, 
1969; Socransky, 1977; Haffajee \& Socransky, 1994). Evidence suggests that specific micro-organisms have been implicated as playing an important role in the breakdown of periodontal tissue (Armitage et al., 1982; Moore, 1987; Slots \& Listgarten, 1988). The 'Specific plaque hypothesis', was put forward by Loesche in 1976. He suggested that only a small number of species of microorganism within the bacterial mass are the main aetiological factor in gingivitis and periodontitis. Furthermore, Socransky (1977) demonstrated that specific groups of bacteria are associated with specific clinical forms of periodontal disease. With regard to this hypothesis, interest has grown regarding the use of antimicrobial therapy for periodontal disease as a means of reducing the level of pathogenic bacteria in the subgingival area.

There are two approaches which can be taken to make the subgingival area less pathogenic. The first would be to reduce the total microbial mass. The use of non-specific antiseptic agents against the plaque microflora and mechanical debridement of the root surface would serve this purpose. The other approach would be to reduce the level of pathogenic micro-organisms in the plaque. To this end, antibiotics would be considered to be the most effective choice. 
Microbiological parameters clearly revealed that chemotherapy directed towards the anaerobic micro-organisms in the subgingival plaque substantially reduced it's pathologic potential (slots et al., 1979; Lundstörm, Johansson \& Hamp, 1984; Loesche et al., 1984). Four principle groups of antibiotics have been extensively tested in the treatment of periodontal disease; these are the penicillins, clindamycin, metronidazole and the tetracyclines.

The penicillins and clindamycin have found only limited application in the control of periodontal inflammation (Slots \& Rams, 1990).

Penicillins (penicillin v, ampicillin, amoxycillin,...) are the antibiotics of choice for premedication as well as for nonperiodontal oral infection. Amoxycillin and ampicillin have proved useful in the treatment of acute necrotising ulcerative gingivitis and severe periodontal disease (Van Winkelhoff et al., 1989). Augmentin (amoxycillin and clavulanic acid) has shown promise in the treatment of refractory periodontitis (Kornman et al., 1989). The major disadvantages of penicillins are the high incidence of 
immediate hypersensivity type allergic reactions and the development of penicillin resistant micro-organisms.

Clindamycin has been shown to be useful for the treatment of refractory periodontitis (Gordon et al., 1990; Walker \& Gordon, 1990). An important complication occasionally seen with clindamycin is the development of pseudomembranous colitis.

Metronidazole and tetracyclines have been extensively used in the treatment of periodontal disease.

\section{Metronidazole}

Metronidazole is a nitromidazole with a narrow range of activity against anaerobes, such as Bacteroides species and spirochetes, which are generally believed to be the major pathogens associated with periodontitis. Metronidazole is bactericidal at low concentrations (Sutter, Jones \& Ghoneim, 1983; Wade \& Addy 1987; Walker et al., 1985). It was discovered in France during the 1950s (Scully, 1988). In the 1962, it was confirmed to be effective in the treatment of Trichomonas vaginalis infection, and ulcerative gingivitis (Shinn, 1962). Subsequently, it was reported that metronidazole killed spirochetes (Davies, MacFadzen \& Squires, 1964). In the early 1970s, investigators observed that it was bactericidal for 
obligate anaerobes (Tally, Sutter \& Finegold, 1972). Since periodontal disease was often associated with anaerobic bacteria, metronidazole was assessed alone and in conjunction with conventional therapy to determine if its use could improve periodontal status (Jenkins et al., 1989; Gusberti, Syed \& Lang, 1988; Soder et al., 1990; Loesche et al., 1992).

The principal members of this group are metronidazole, tinidazole and ornidazole, and they have been prescribed alone, or in combination with other antibiotics, for example, amoxicillin (Van Winkelhoff et al., 1989; Van Winkelhoff, Tijhof \& de Graaff, 1992) or augmentin (Kornman et al., 1989) in the treatment of severe infections. Various combinations of metronidazole have been found to have an anti-plaque potential. There has been little evidence of tinidazoles efficacy in the treatment of chronic periodontitis. Metronidazole is well absorbed after oral administration and penetrates into body tissues, cerebrospinal fluid, saliva and can be detected in the gingival crevicular fluid (GCF) (Britt \& Pohlod, 1986). It is metabolised in the liver, the unchanged drug and the metabolites are excreted in the urine (Plaisance, Quintilian \& Nightingale, 1988). Concurrent food ingestion does not inhibit bioavailability; however absorption may be slowed. Britt and Pohlod (1986) reported that the concentration found in the GCF is only half that found in 
the serum. Metronidazole gains access to aerobic and anaerobic bacterial cells by diffusion.

Metronidazole has been used orally in tablet form and for topical application. Various dosages and durations of metronidazole therapy have been used in the treatment of periodontal patients. The most commonly prescribed drug regimen was 200 to $250 \mathrm{mg}$ three times a day (tid) for 7 days (Jenkins et al., 1989; Loesche et al., 1991, 1992). Local application of metronidazole in devices such as pulsed-jet irrigators, acrylic strips, dialysis tubing, subgingival displacement pressure packs and dental paste was found effective as an adjunct to conventional treatment (Aziz-Gandour \& Newman, 1986; Khoo \& Newman 1983; Newman et al., 1984; Addy, Langeroudi \& Hassan, 1985; Addy et al., 1988; Yeung, Newman \& Addy, 1983; Klinge et al., 1992).

Numerous side effects have been recorded which are not often serious. These are gastrointestinal discomfort, diarrhoea, nausea, loss of appetite, a bitter and metallic test, urticaria, and discoloured urine. Dizziness and headaches have also been reported. Occasionally skin rashes have occurred. Patients who are prescribed metronidazole, should be instructed not to take alcohol as the drug may inhibit alcohol metabolism. Patients undergoing anticoagulant therapy can be affected by metronidazole because it inhibits warfarin metabolism, 
thereby prolonging prothrombin time. Similarly, individuals taking lithium should also avoid metronidazole, because even short-term usage of metronidazole can cause elevated serum lithium levels. Administration of metronidazole in pregnancy should be avoided especially in the first trimester.

\section{Tetracyclines}

The tetracyclines are broad-spectrum bacteriostatic antibiotic agents which are effective against a number of oral Gram-negative and Gram-positive cocci and bacilli. Tetracyclines are also effective against many anaerobic and facultative bacteria, which is especially relevant from a periodontal standpoint. They are also active against most spirochetes. Chlortetracycline was the first tetracycline isolated from the fermentation products of streptomycin aureofaciencs which were initially obtained from soil micro-organisms. They were introduced into clinical practice in 1948 (Duggar, 1948). Tetracyclines have also been used in dentistry, particularly as adjuncts to periodontal therapy (Reviewed by Seymour \& Heasman, 1995). There are several different kinds of tetracyclines available on the market. Tetracycline hydrochloride, doxycycline and minocycline which are all semisynthetic tetracyclines, are used extensively in the treatment of periodontal disease. 
The tetracyclines, especially minocycline and doxycycline, are lipophilic and are thus highly diffusible, resulting in their antibacterial action, and also reflecting their ability to penetrate developing dental structures and bone and also enter the cerebrospinal fluid, and crevicular fluid. The tetracyclines, minocycline, and doxycycline are detectable in the GCF after oral administration and their concentrations can reach levels of 10 times and 5 times respectively, those in serum. This is of particular importance when the drugs are used for the treatment of periodontal disease. Minocycline has a longer serum halflife and a lower urinary excretion rate than most other tetracyclines which permits the use of smaller and less frequent doses (Genco, 1981).

Tetracyclines are absorbed from the gastrointestinal tract. Absorption is fairly rapid, but significant amounts are retained in the bowel. The absorption of tetracycline is reduced when the drugs are taken with milk products or with substances containing calcium, magnesium, iron, or aluminium. Therefore it is advisable to refrain from these products for at least an hour and a half prior to or following administration of the medication by the oral route. However, food does not interfere with the absorption of doxycycline or minocycline. Excretion of the tetracyclines occurs in both the urine and the faeces, but mainly via the kidneys. There is some reabsorption of the 
drug which prolongs their duration of action. Doxycycline and minocycline are excreted predominantly in the faeces. The tetracyclines are bacteriostatic antimicrobials that exert their antibacterial activity by inhibiting microbial protein synthesis (Chopra \& Howe, 1978).

The tetracyclines have been administered both orally and topically in periodontal treatment (Goodson, Haffajee \& Socransky, 1979; Goodson et al., 1983; Lindhe et al., 1979). The most commonly prescribed regimen was 200 to 250 $\mathrm{mg}$ at six-hourly intervals for two weeks (Slots et al., 1979; Rams \& Keyes, 1983) for tetracycline hydrochloride. The adult dosage for doxycycline is a loading dose of 200 $\mathrm{mg}$, followed by $100 \mathrm{mg} /$ day for 7 days; for minocycline is $100 \mathrm{mg}$ twice a day for a week. Tetracyclines have been applied in different delivery devices such as cellulose acetate fibres (Lindhe et al., 1979), hollow fibres (Goodson, Haffajee \& Socransky, 1979; Lindhe et al., 1979), ethyl cellulose fibres (Friedman \& Golomb, 1982), acrylic strips (Addy et al., 1988; Addy, Langerondi \& Hassan 1985), collagen preparations (Minabe et al., 1989, 1991), white petrolatum (Eckles et al., 1990), inserts of polymethacrylic acid with hydroxypropylcellulose (Higashi et al., 1990), hydroxypropylcellulose films (Noguchi et al., 1984), powder (Okuda et al., 1992), ointment (Rosania \& Kornman, 1981), solution (MacAlpine et al., 1985; Puchalsky et al., 1988), gel (Van Steenberghe et al., 
1993), ethylene vinyl acetate copolymer (EVA) fibres (Goodson et al., 1983).

The side effects of tetracyclines are hypersensitivity reactions, gastrointestinal disturbances, nausea, vomiting or diarrhoea, and pseudomembranous colitis. Candida albicans has also been reported in patients who have received a long course of treatment with tetracycline. Large doses of tetracyclines given parenterally can damage the liver; pregnant woman appear to be particularly susceptible. Immediately after absorption, tetracyclines are built into calcifying tissue and become a permanent, discolouring feature of the teeth, if administered to children under 12 years of age. The use of tetracyclines should be avoided during the period of crown formation of the teeth.

$1.8 \cdot 4$

Local versus systemic delivery of antibiotics in periodontitis therapy

The purpose of using an antimicrobial is to achieve a sufficient concentration of the drug in the periodontal environment to kill or arrest the growth of pathogenic micro-organisms. With systemic antibiotic therapy the concentration at the site of infection is dependent on the level achieved in the serum. The concentration at the site 
of infection is relatively low, because the administered dose is distributed throughout the blood stream. Several studies have shown that the concentration of some antibiotics in the GCF is equal to or 10 times more than that of the serum. However, local antibiotic administration can accomplish 100 fold concentrations at subgingival sites than can be achieved by systemic therapy (Goodson et al., 1985a). One of the principal aims of current periodontal antibiotic research is to reduce the need for systemic prescription with the disadvantage of potential adverse side-effects. This becomes even more relevant when considering evidence that crevicular fluid levels achieved using local antibiotic therapy were adequate to inhibit all cultivable organisms in a given subgingival plaque (Wilson, O'Connor \& Newman, 1990, 1991). Furthermore, local therapy enables the utilisation of antimicrobial agents which do not lend themselves to systemic administration. Subgingival irrigation suppresses the subgingival microbiota for at least a short period of time.

\subsubsection{Clinical efficacy of antibiotics in periodontal disease}

Several studies compared the ability of antibiotic therapy alone to favourably alter clinical and microbiologic 
parameters, with root planing. These investigations indicated that drug therapy alone was inferior to mechanical instrumentation (Lindhe, Liljenberg \& Adielson, 1983; Walsh et al., 1986; Lekovic et al., 1983). With regard to the above conclusion, several further studies suggested the use of antibiotics as an adjunct to conventional methods in the treatment of periodontal lesions (Loesche et al., 1981, 1984; Lindhe et al., 1982; Lundström, Johansson \& Hamp, 1984; Soder et al., 1990). The results from clinical trials using antibiotic therapy alone and as an adjunct to conventional therapy are controversial. Jenkins et al. (1988) concluded that antibiotic therapy could not be justified in patients with poor plaque control.

A 6 month multicenter clinical trial of 206 patients was conducted comparing metronidazole gel application to scaling using a split-mouth design. The results of this study suggested that the use of the gel alone without any mechanical root-instrumentation reduced pocket depth by 1.3 mm, however, subgingival root planing gave a statistically better reduction (Ainamo et al., 1992). The effect of a 1\% metronidazole gel was evaluated in 10 patients with periodontitis during the maintenance phase as an adjunct to scaling and root planing (Needleman \& Watts, 1989). There was no significant difference in probing depth or attachment level between teeth treated with $1 \%$ 
metronidazole gel versus teeth treated with a placebo gel at 1 and at 3 months post treatment. The results of a 3 month study of 28 patients which compared the metronidazole gel application plus scaling to scaling alone using a split-mouth design, failed to show a significant pocket depth reduction, or attachment gain between test and control groups (Hitzig et al., 1994).

A 12 month study was carried out in 122 patients, comparing tetracycline fibres alone to scaling and root planing plus tetracycline fibres. This method of slow release of tetracycline into the periodontal pocket was shown to be effective (Goodson, Haffajee \& Socransky, 1979). Analysis of data from 107 subjects also indicated that tetracycline fibre therapy significantly decreased pocket depth, increased attachment level, and decreased bleeding on probing in two months (Goodson et al., 1991). In a 6 month study comparing scaling and root planing and tetracycline fibres used together with scaling alone, a 40\% improvement in pocket depth and attachment level was found in the former group. The fibres used in conjunction with scaling and root planing produced a statistically significant improvement in pocket depth reduction, attachment level gain and bleeding on probing reduction at 6 months after therapy (Kornman et al., 1993) 
Van Steenberghe et al. (1993) reported a study comparing the effect of minocycline gel plus scaling and root planing to a placebo gel plus scaling and root planing, in 103 patients, at one and three months from baseline. Patients were randomly assigned to 2 groups in a parallel design. There was a statistically significant difference between minocycline gel and the placebo gel, in terms of pocket depth reduction, at 3 months after treatment $(p<0.002)$. There was no significant different between minocycline gel and the placebo gel in gain in probing attachment level and reduction of bleeding index. There was a significant reduction in Actinobacillus actinomycetemcomitans (Aa), Porphyromonas gingivalis (Pg), and Prevotella intermedia (Pi) for minocycline treated teeth at 3 months after treatment.

Murayama et al. (1989) have demonstrated that following the treatment of sixteen subjects with scaling and the application of $2 \%$ minocycline gel there was a decrease in plaque accumulation, gingival inflammation, pocket depth, bleeding on probing and also a shift of the microflora to one associated with healthy gingivae for at least 11 weeks. Locally applied minocycline can also prevent bone loss (Toth et al., 1986). 
The tetracyclines are now known to have non-antimicrobial properties that may also magnify their therapeutic effect. These properties are: a) their abilities to bind to the tooth surface and then be slowly released in an active form over a prolonged period of time (Baker et al., 1983), which could prolong their therapeutic effectiveness after the patient has stopped taking the drug; b) their antiinflammatory action which might be related to the ability of tetracyclines to suppress PMN migration, degranulation and oxygen radical synthesis, thereby reducing PMN-related tissue damage, while simultaneously inhibiting disease associated species (Martin et al., 1974; Gabler \& Creamer 1991); c) their ability to promote the attachment of fibroblasts and connective tissue to root surfaces (Somerman et al., 1988; Rompen et al., 1993); d) their ability to inhibit bone resorption (Golub et al., 1984; 1991; Gomes, Golub \& Ramamurthy, 1984); e) their unique ability among antibiotics to directly inhibit collagenolytic enzymes; f) their ability to recognise the cellular source of collagenase in the inflammatory exudate from human periodontal pockets, and thereby inhibit its activity is, an additional therapeutic role in periodontal disease, and is able to used as a diagnostic probe (Suomalainen et al., 1992). 
Teng et al. (1992) showed that metronidazole treatment can cause the reduction of both active and latent forms of gelatinase. They reported that treatment of patients with recurrent loss of periodontal attachment, with metronidazole $(250 \mathrm{mg}$, tid, for 7 days $)$ significantly reduced the level of active and latent gelatinase by 4 - to 6 fold $(p<0.002)$. They concluded that the reduction of gelatinase activity is best explained by the antimicrobial effect of metronidazole which appears to blunt the destructive part of the host immune and inflammatory response.

1.8 .6 .1 Anti-inflammatory effects of tetracyclines

Potential anti-inflammatory properties of tetracyclines (Gabler \& Creamer, 1991) appear effective in the treatment of specific skin disorders which do not have a bacterial aetiology. The efficacy of the tetracyclines in the treatment of these diseases may be related to their general anti-proteolytic properties or to some anti-inflammatory properties. Several non-antimicrobial mechanisms may be involved, including tetracycline's ability, to inhibit prostaglandin production, to suppress leukocyte activity and to function as scavengers of superoxide radicals. Chang and Houck (1970) reported that collagen breakdown products are chemotactic for polymorphonuclear leukocytes 
(PMNS). Galber and Creamer (1991) suggested that suppressed collagenase activity in the gingival crevicular fluid of tetracycline treated individuals might also result from a decrease in oxidant-mediated activation of latent neutrophil collagenase. It was also suggested that inhibiting the generation of arachidonic acid by blocking phospholipase A2 activity (Pruzanski et al., 1992), and blocking the synthesis of prostaglandin $E_{2}\left(P E_{2}\right)$ (El-Attar, Lin \& Shultz, 1988) may be related to anti-inflammatory properties of the tetracyclines in the treatment of disease.

1.8 .6 .2

Anti-collagenase activities of tetracyclines

The potential anti-collagenase activity of the tetracyclines was verified when Golub et al. produced a chemically modified tetracycline molecule (CMT) whose antimicrobial efficacy was eliminated, but which did retain its ability to inhibit collagenase. They also noted the ability of tetracyclines to reduce diabetic gingival collagenase activity in germ-free rates (Golub et al., 1983). Because of these effects in germ-free rats, it was proposed that tetracyclines could inhibit extracellular collagenase by a mechanism independent of the drugs' antibacterial activity. 
Tetracyclines have been found to inhibit collagenases from different tissues and cellular sources, including PMNs (Golub et al., 1983, 1985a,b), macrophages (Maehara et al., 1988), osteoblasts (Ramamurthy et al., 1993), chondrocytes (Greenwald et al., 1987), and tumor cells (Zucker et al., 1989) as well as extracts of human gingiva (Golub et al., 1990; Yanagimura et al., 1989) and synovial tissue (Greenwald et al., 1987).

Recent studies indicate that tetracycline inhibition of collagenase activity differs between different tissues and between cells from the same tissue, and, second, members of the tetracycline family of compounds may also differ in their anticollagenase effectiveness (Maehera et al., 1988; Lee et al., 1991). For example, clonal URM 106-01 rat osteoblastic gelatinase is inhibited by exposure to tetracycline, whereas transformed rat osteoblastic osteosarcoma gelatinase is not sensitive to the drug (Vernillo et al., 1993). Furthermore, human polymorphonuclear leucocyte collagenases are relatively sensitive to tetracycline inhibition compared with human fibroblast collagenases (Golub et al., 1991; Ingman et al., 1993; Suomalainen, et al., 1992; Golub et al., 1995). Pharmacologic concentrations of minocycline (2 to $25 \mu \mathrm{g} / \mathrm{ml}$ ) were found to inhibit crude collagenase extracted from human PMNs, whereas the collagenolytic activity of cultured human fibroblasts was resistant to this effect until 
extremely high concentrations of the drug were used (Golub et al., 1983, 1990; Maehara et al., 1988).

With regard to the fact that members of the tetracycline family differ in their ability to inhibit extracellular collagenase activity, Golub et al. (1985a) reported that administration of minocycline or doxycycline to humans with periodontal disease appeared to inhibit collagenase in the periodontal pocket for a longer period of time than tetracycline. In addition Golub et al. (1991, 1992) and Yangamura et al. (1989) also demonstrated that doxycycline and minocycline are more potent than tetracycline HCL. Burns et al. (1989) confirmed the above result and proposed that the greater effect of doxycycline was related to its stronger binding of the zinc cation at the active site of the enzyme.

Several studies have shown that tetracyclines act on both the latent and active forms of collagenase (Greenwald et al., 1987; Mcculloch et al., 1990; Lee et al., 1991).

So far, several mechanisms of action of these drugs have been identified including: a) their ability to block already-active MMPs presumably by binding to the $\mathrm{Zn}^{2+}$ cation in the enzyme molecule. It has been suggested that tetracyclines bind to a secondary zinc (or $\mathrm{Ca}^{+2}$ ) cation not at an active site in the enzyme, resulting in a 
conformational change and loss of catalytic activity of the MMP (Lovejoy et al., 1994); b) their ability to prevent the oxidative activation of latent procollagenase and progelatinase secreted by several cell types (PMN leukocytes, osteoblasts) found in the periodontium (Lauhio et al., 1992; Ramamurthy et al., 1993); c) their ability to convert proenzyme to smaller inactive fragments; d) their ability to decrease synthesis of Pro-MMPs (Uitto et al., 1994; Rifkin, Vernillo \& Golub, 1993); e) their ability to protect endogenous MMP inhibitors (TIMP-1) (Sorsa et al., 1993 ) and other neutral proteinase inhibitors ( $\alpha 1$ antitrypsin) (Sorsa et al., 1993) from degradation and inactivation, because of reduced elastase activity in vivo. Elastase has recently been observed to degrade TIMPs (Okada et al., 1988). Tetracyclines might also prevent the oxidative inactivation of TIMPs by reactive oxygen species such as HOCL (Lauhio et al., 1992; Strickling \& Hoidal 1992); f) their ability to contribute indirectly to inhibition of additional MMPs, i.e., stromelysin and elastase, which may not be directly inhibited by these drugs; g) their ability to enhance production of collagen (Schneir et al., 1990; Sasaki et al., 1992a) and bone (Sasaki et al., 1992b) when these processes are suppressed during disease such as in diabetes-induced skin lesions and periodontal ligament atrophy and osteopenia. 
With regard to the effects of tetracyclines on production, activity, and regulation of MMPs, and also the effects of locally delivered antibiotics in the clinical treatment of periodontal disease, it was decided to examine the effect of locally delivered antimicrobial systems, on the levels of stromelysin and TIMP in sites which did not respond to conventional treatment. 


\section{CHAPTER 2}

\section{MATERIALS \& METHODS}


Prior to the commencement of these studies ethical approval was obtained from the Glasgow Dental Hospital Ethics Committee. Subjects participating in these studies were informed of the protocol and consent was obtained. All patients taking part in the longitudinal investigation were free to withdraw from the study at any time.

The study population consisted of patients who had been treated by means of subgingival scaling and root planing but still displayed signs of persistent periodontal disease, defined as residual pocketing of $\geq 5 \mathrm{~mm}$, with bleeding on probing (BOP) or suppuration. Fifty two subjects fulfilling the above criteria were selected from patients in the Department of Periodontology, Glasgow Dental Hospital and School. The subjects had no history of systemic disease which could influence the course of periodontal treatment. They were not on any medications that could influence the manifestations of periodontal disease, and they had not received antibiotic therapy within the past 6 months.

Thirty two of the subjects were female and twenty were male, with a mean age of 45, ranging from 30-67. 
Site selection was carried out at a screening visit, after a full mouth periodontal examination using a CP-12 periodontal probe (Ash, Densply, UK). Four non-adjacent sites with periodontal pockets equal to or deeper than $5 \mathrm{~mm}$ with bleeding on probing or suppuration were selected. In order to increase the accuracy of the measurements taken and to simplify the harvesting of GCF, the buccal, anterior, mesial sites were preferred to the lingual, posterior, and distal sites.

\author{
Longitudinal study of the effects of \\ adjunctive locally delivered antibiotic \\ therapy on matrix metalloproteinase and \\ inhibitor in gingival crevicular fluid
}

The patients in this study were seen at the following visits: 1) screening visit, 2) baseline measurements visit, 3) treatment visit, 4) reassessment visit. At the screening visit, all study subjects signed an informed consent form. A full medical history was recorded, and a full periodontal examination was carried out. If the patient fulfilled the criteria, (section 2.2) alginate impressions were taken of 
the upper and lower jaws, and an acrylic stent (Kombiplast, Dreve-Dentamid-GmbH) fabricated for attachment level measurements.

Oral hygiene instruction was given according to individual needs, but no periodontal instrumentation was carried out. 10 days after the screening visit baseline measurements were taken in the following order:

1) Plaque Index (PI) (Silness and Löe, 1964) and Modified Gingival Index (MGI) (Lobene et al., 1986) were recorded for the study sites.

2) GCF samples were taken from the study sites and GCF volume recorded with the periotron.

3) Bleeding on probing (BOP) and suppuration scores were recorded from the preselected sites.

4) The first set of pocket depth (PD) measurements was performed with the electronic pressure sensitive probe.

5) The first set of attachment measurements of the study sites was made with the acrylic stent and the electronic pressure sensitive probe. 
6) After changing the patient's position and removing and replacing the stent, the second set of pocket depth and attachment level measurements were carried out.

The study had a parallel design. The patients were randomly allocated into four groups, three of which received scaling followed by a locally delivered antimicrobial system. 6 weeks after the last treatment visit, patients were seen for a reassessment visit and the same measurements as at baseline were recorded.

One examiner (MR), carried out all clinical measurements throughout the study. The following parameters were recorded for the patients at baseline and at the 6 week visits. The order in which the measurements were taken, appears below.

The plaque index (PI) of Silness and Löe, (1964) was used for recording plaque accumulation. The scoring system is as follows: 
A film of plaque adhering to the free gingival margin and adjacent surface of the tooth. The plaque may only be noticed by running a probe across the tooth surface.

2

A moderate accumulation of soft deposits within the gingival crevice, on the gingival margin and/or adjacent tooth surface. An abundance of soft matter within the gingival crevice and/or on the gingival margin and adjacent tooth surface.

The Plaque Index assesses very small differences in plaque accumulation (Lindhe, 1989). In this study, site scores refer to a single site $\mathrm{PI}$ recording.

\subsubsection{Modified Gingival Index}

The Modified Gingival Index (MGI) (Lobene et al., 1986) was used to assess gingival inflammation and is described below: 
Mild inflammation; slight change in colour, little change in texture of any portion but not the entire marginal or papillary gingival unit.

Mild inflammation; criteria as above but involving the entire marginal or papillary gingival unit.

Moderate inflammation; glazing, redness, oedema, and/or hypertrophy of the marginal or papillary gingival unit.

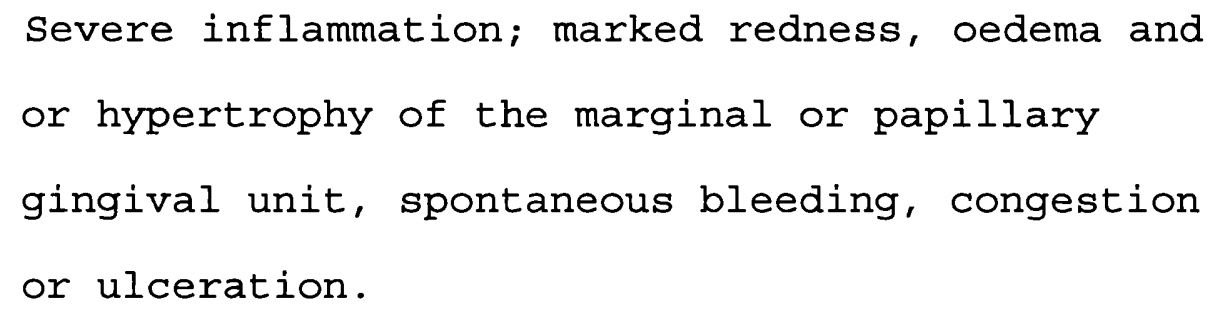

The MGI was preferred to the more commonly used Gingival Index (Löe \& Silness, 1963) because it provides greater sensitivity to the earliest changes of gingivitis as the lower part of the scale is expanded resulting in a scoring system of $0-4$. Since bleeding upon probing is not a criteria in the MGI, it is a completely non-invasive procedure. A non-invasive method was essential in this study since GCF samples were taken after the MGI scoring, 
and procedures which might irritate the gingival tissue and thereby alter the GCF constituent profile had to be avoided.

2.4 .3

The gingival crevicular fluid sampling method

Sample collection was performed in duplicate with a one minute interval, using filter paper strips and the fluid volume on the strip was measured immediately after sampling with a Periotron 6000 (Harco Electronic, Winnipeg, Canada) .

In the clinic, GCF was sampled after the PI and MGI scores were taken but before any other clinical recordings which could cause irritation of the tissues and serum contamination of the sample.

The site to be sampled was isolated with cotton wool rolls and supragingival plaque was carefully removed. The region was dried with a gentle air stream and 30 second (s) later GCF was collected with a Whatman grade 4 paper strip (Whatman Labsales Ltd., Maidstone, Kent) $(2 \times 13 \mathrm{~mm}$ ) (Griffiths et al., 1988) inserted into the crevice until mild resistance was felt and left there for 30 secend (Figure 2.1). Strips visually contaminated with saliva or blood were discarded. The sampling method is reliable and 


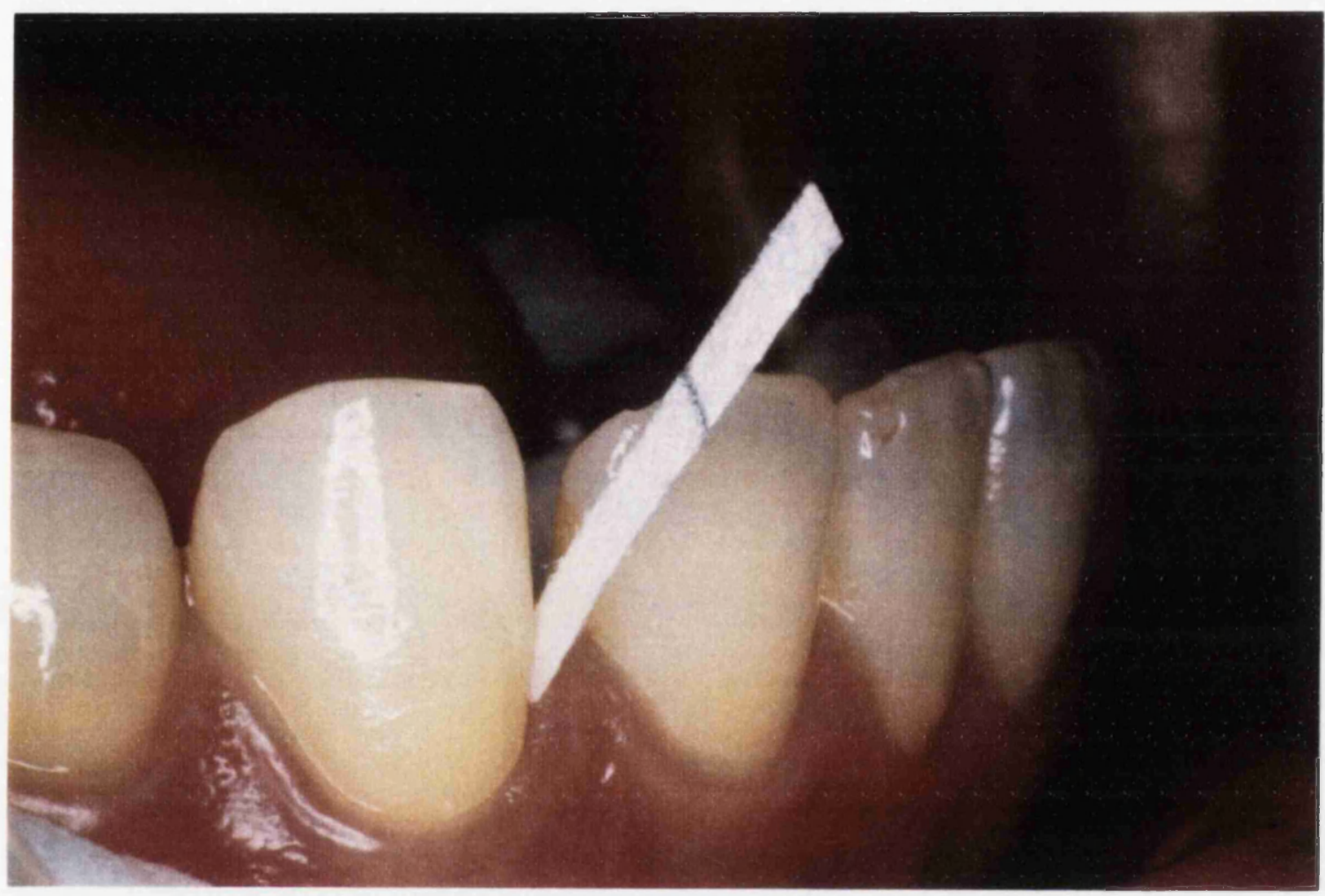

Figure 2.1 Gingival crevicular fluid sampling 
causes no significant disturbance of the gingival blood vessels (Gustafsson et al., 1992). The fluid volume on the strip was measured immediately after sampling with a Periotron 6000 GCF (Harco Electronic, Winnipeg, Canada) (Figure 2.2). The jaws of the periotron were wiped with pure ethanol and then dried between readings. The strips were then placed in individual sterile tubes and stored at -30 centigrade degree $\left({ }^{\circ} \mathrm{C}\right)$ until further processing was carried out. Subsequently, the strips were eluated into 500 microlitres $(\mu l)$ of incubation buffer at room temperature using a rotary mixer. The strips were then discarded and the elute aliquoted in sterile tubes and stored at $-30^{\circ} \mathrm{C}$. These aliquots were subsequently analysed for the quantification of SL and TIMP.

\subsubsection{Bleeding on probing}

The Florida Probe was used to assess bleeding upon probing at the same time as the first set of probing pocket depth measurements. BOP scoring was carried out within 30 s of probing. 


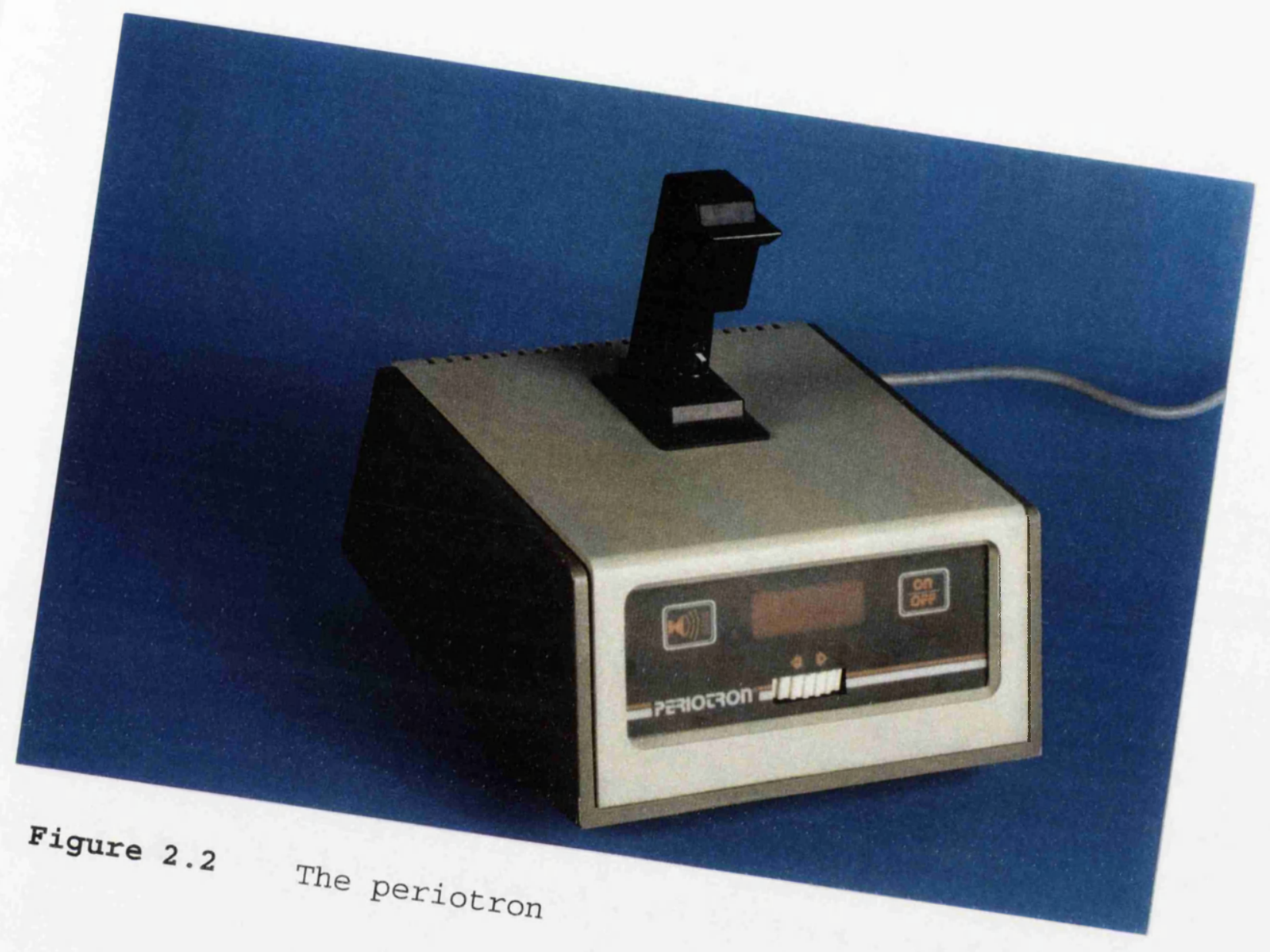


A ball burnisher was used to apply gentle pressure on the gingival wall of the periodontal pocket in an apico-coronal direction in order to determine the presence or absence of suppuration.

\subsubsection{Probing depth and attachment level assessments}

In this study pocket depth and attachment level (AL) were recorded to the nearest 0.2 millimetre $(\mathrm{mm})$ using the Florida probe (Florida Probe Corporation, Florida, USA) (Gibbs et al., 1988). The Florida Probe is an electronic pressure sensitive probe with a constant force of 20 gram (g). The system consists of a pocket depth and an attachment level ('stent') handpiece, a foot switch, a computer interface and a portable personal computer (Figure 2.3). When the foot switch is pressed, the measurements of $\mathrm{PD}$ and $\mathrm{AL}$ are made electronically, using the 'pocket depth' and 'attachment level' stent. Measurements were recorded by an assistant from the computer screen, as the operator was blind to the measurement. The probe tip has a diameter of $0.4 \mathrm{~mm}$, no visible graduations along its length and it reciprocates through a sleeve. The edge of the sleeve is the reference from which measurements are recorded. As 


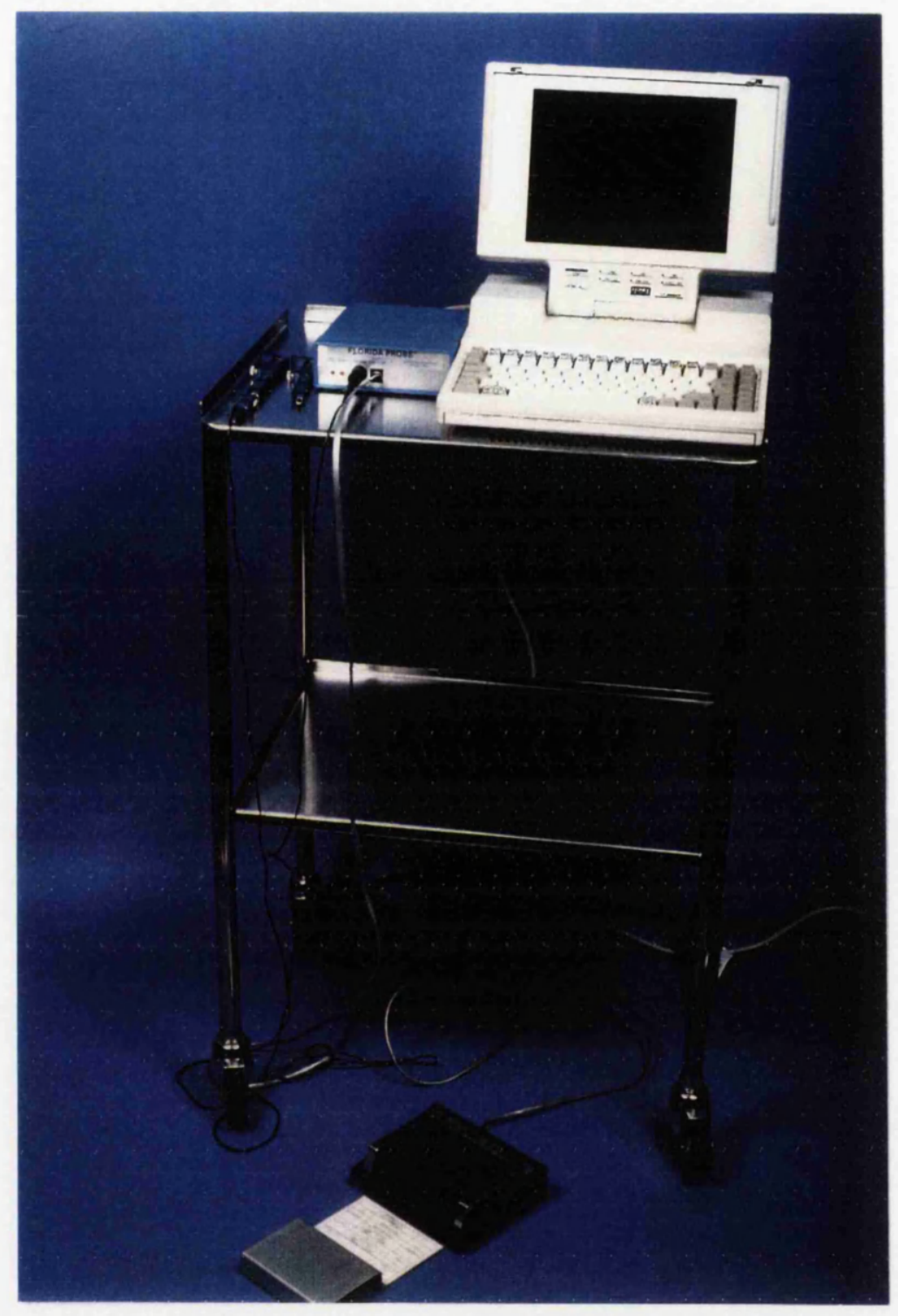

Figure 2.3 Florida probe; consists a computer interface and a portable personal computer, a foot switch, pocket depth and an attachment level ('stent') handpiece 
identification of the cemento enamel junction (CEJ) is often complicated by its subgingival location or the presence of restorations, the following method of recording attachment level measurements was used. A fixed reference point is required for attachment level measurements. The Florida probe 'stent' handpiece has a $2 \mathrm{~mm}$ diameter collar at the edge of its sleeve which, during attachment level measurement, is seated on a custom made hard/soft acrylic stent (Kombiplast, Dreve-Dentamid-GmbH) covering the crowns of the teeth. These stents were constructed for both upper and lower arches for each patient from a $2 \mathrm{~mm}$ thick silicone layer using a modification of the method described by Isidor, Karring and Attstrom, (1984). The stents were made on individually cast models using a vacuum forming unit. The stents were then trimmed to cover approximately the coronal one third of the crowns of the teeth at the sites selected for the study. At other sites as much as possible of the stent was preserved in order to assist location and retention. Using an inverted cone bur and a low speed handpiece grooves approximately $1 \mathrm{~mm}$ deep, $2 \mathrm{~mm}$ wide and $1 \mathrm{~mm}$ high were cut, about $1 \mathrm{~mm}$ away from the apical edge of the stent, at the sites to be used in the study. The grooves were then marked with a permanent pen for ease of identification. The disc of the probe handpiece was located in these grooves during AL measurements. Attachment level was recorded relative to the point at which the probe handpiece disc was seated on 


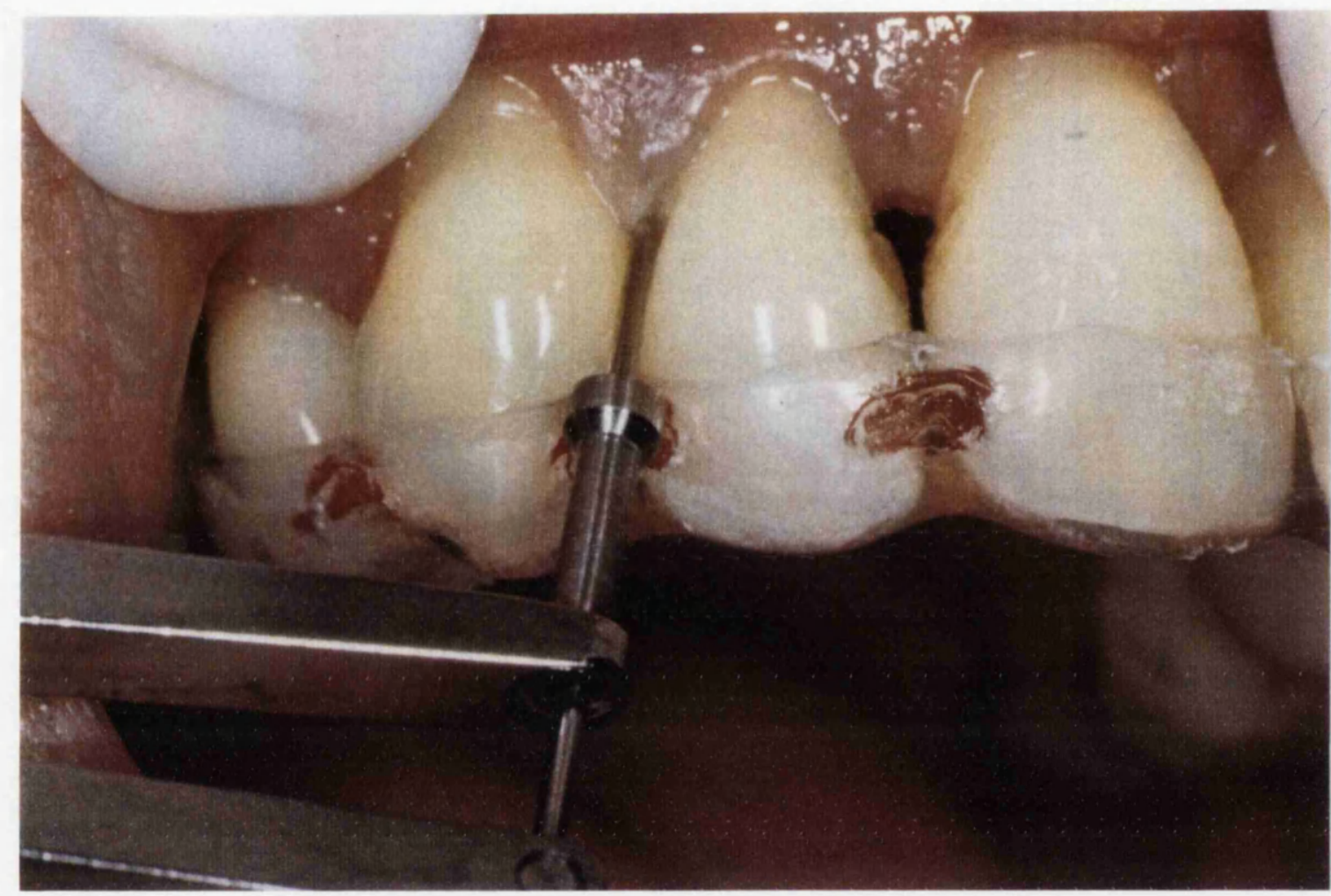

Figure 2.4 Attachment level measurement using the Florida probe and occlusal stent 


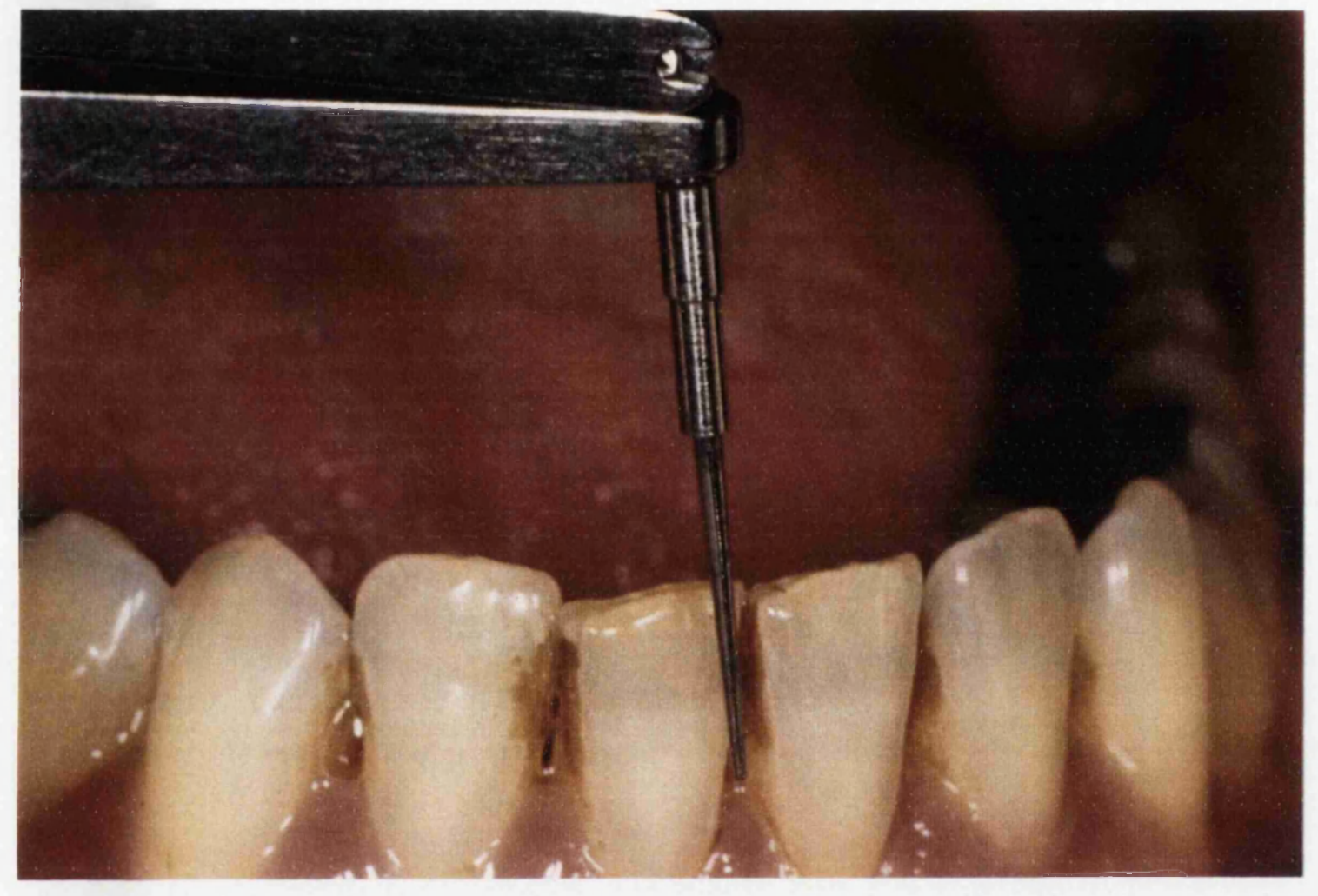

Figure 2.5 Probing depth measurement with the Florida probe 
the stent (Figure 2.4). The grooves on the stent were located to record interproximal $\mathrm{AL}$ at the mid point between the contact point and the adjacent line angle.

PD was assessed by using the point at which the probe sleeve was brought into contact with the gingival margin and was at the same point as the measurement was recorded (AL recording). Care was taken to ensure that the probe tip was held parallel to the long axis of the tooth (Figure 2.5).

$\mathrm{PD}$ and $\mathrm{AL}$ measurements were taken in duplicate. After the first set of measurements was obtained, the stents were removed, the patient's position was changed, and the examiner changed his position by leaving the examination room. For the second set of measurements all the procedures were repeated.

A single operator (NP), performed all the clinical treatment throughout the study. At the treatment visits each group received one of the following treatments:

1) Subgingival scaling and root planing under local anaesthesia followed by application of $0.5 \mathrm{~mm}$ diameter 
ethylene vinyl acetate copolymer fibres containing 25\% tetracycline hydrochloride (Actisite, ALZA, corporation, Palo Alto, CA), into the pocket (Figure 2.6).

2) Subgingival scaling and root planing under local anaesthesia followed by application of $2 \%$ minocycline hydrochloride ointment (Dentomycin, Cyanamid international, Lederle Division, Wayne, NJ) into the pocket (Figure 2.7).

3) Subgingival scaling and root planing under local anaesthesia followed by application of metronidazole $25 \%$ dental gel (Elyzol, Dumex, Denmark) into the pocket (Figure 2.8).

4) The control group received only subgingival scaling and root planing under local anaesthesia.

At each visit the treated sites were examined for any signs of tissue reaction. The patient was asked whether any symptoms had been noted. 42 days post treatment all the baseline measurements were repeated for each patient. During this study other sites received conventional maintenance care. 


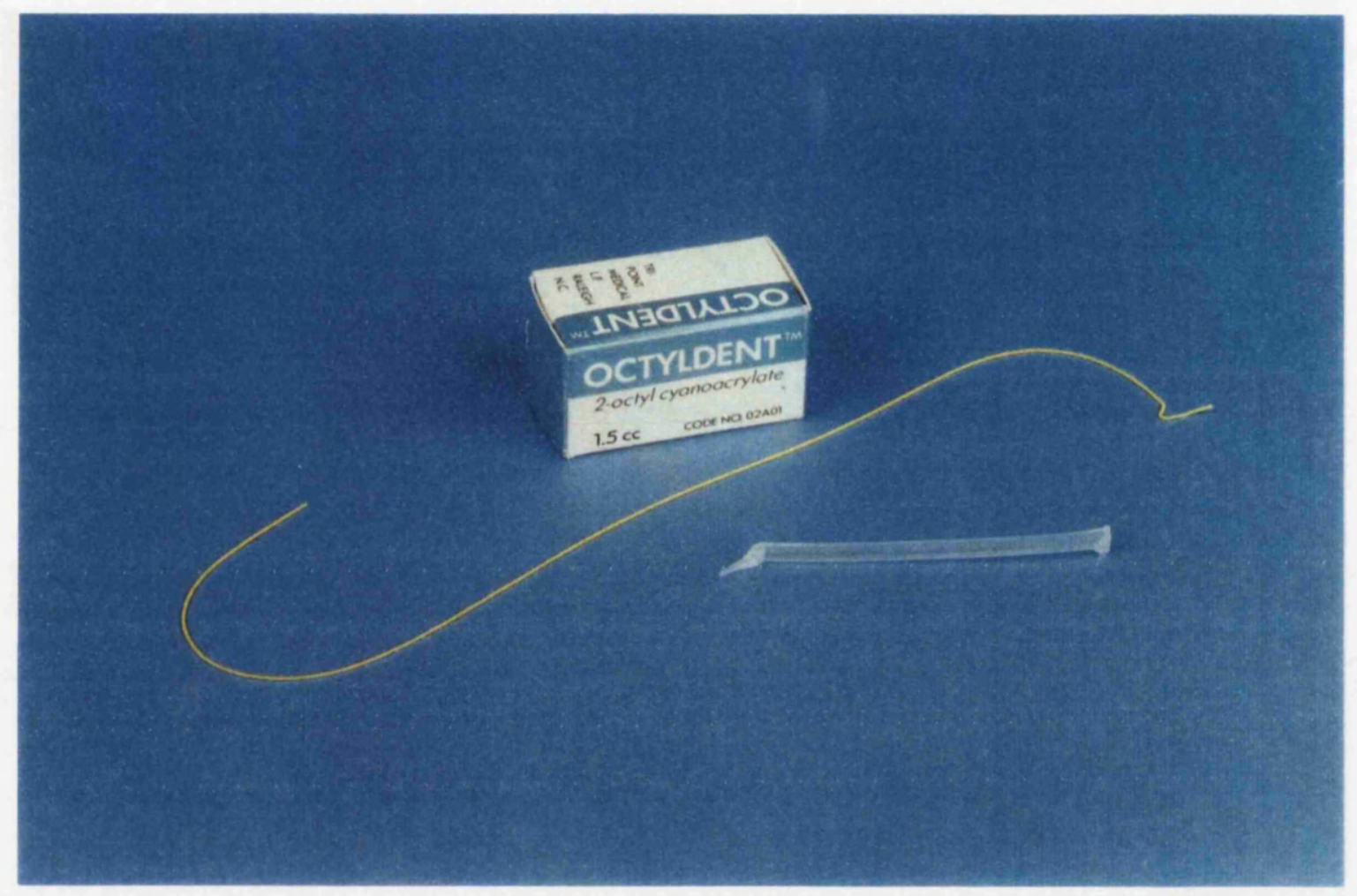

Figure 2.6 Tetracycline fibre and cyanoacrylate adhesive (Actisite) 


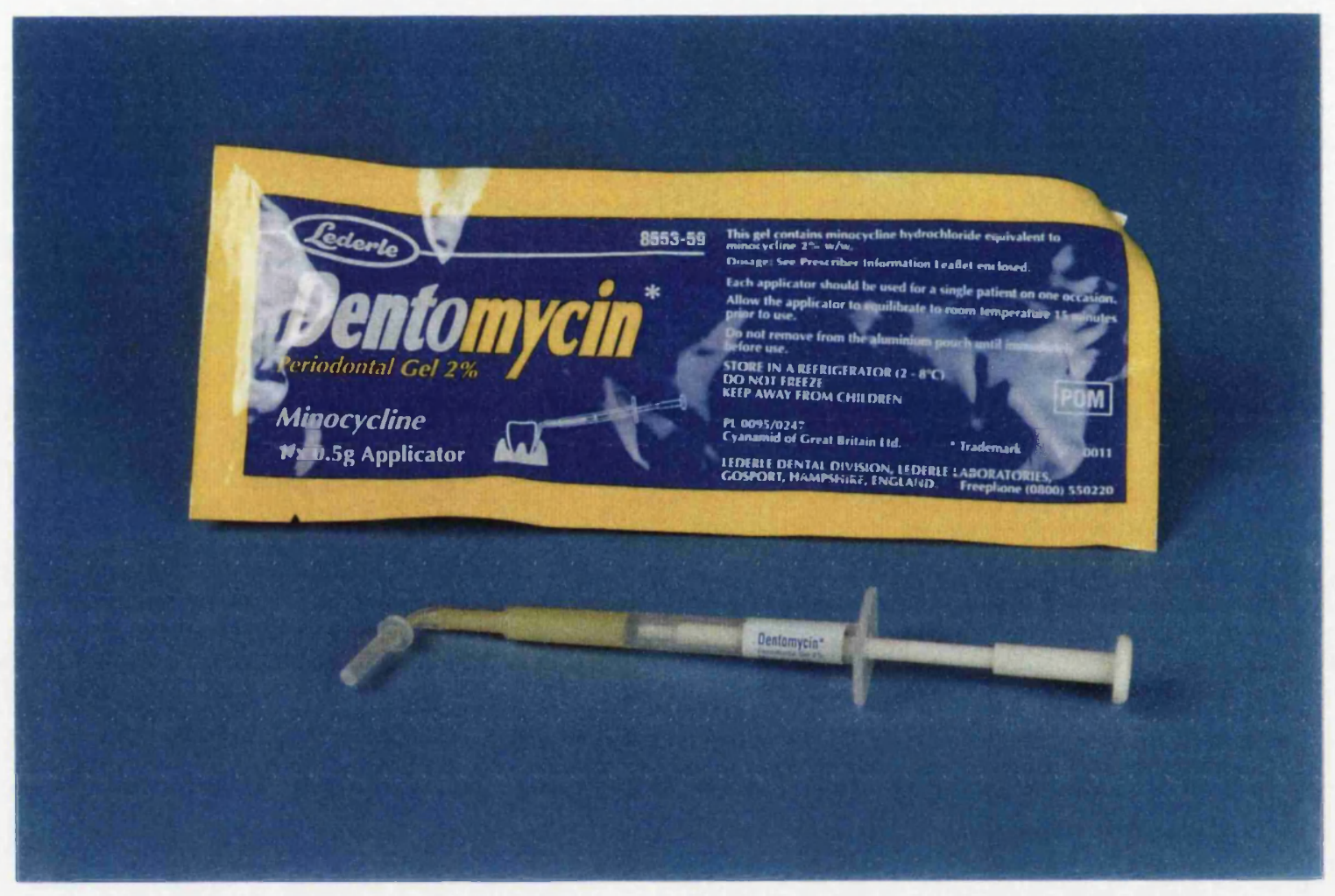

Figure 2.7 Minocycline gel (Dentomycin) 


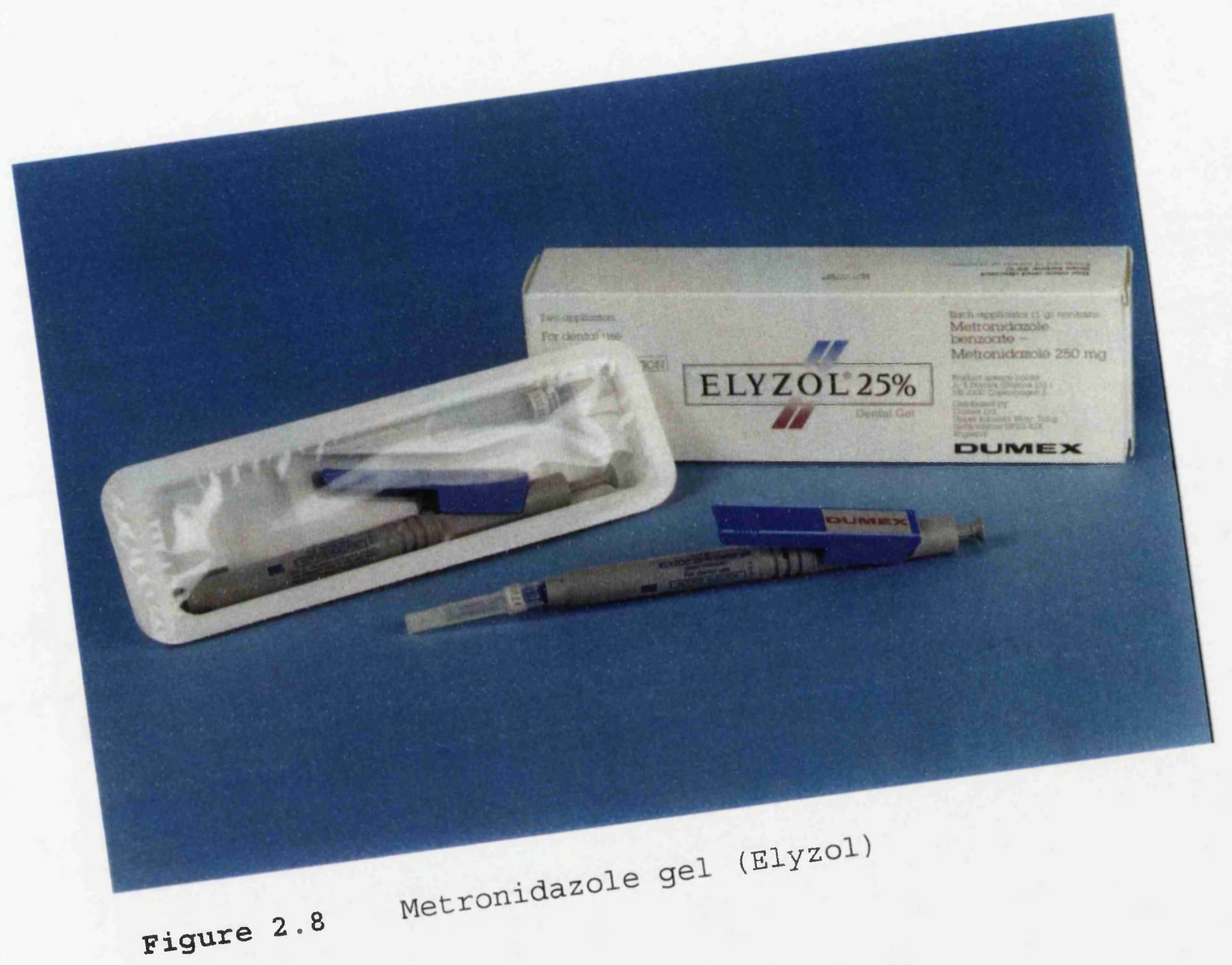


Tetracycline fibre is a $0.5 \mathrm{~mm}$ diameter fibre made of ethylene vinyl acetate copolymer, which is loaded $25 \%$ by weight with tetracycline hydrochloride. The fibre contains 0.5 milligram (mg) tetracycline per centimetre (cm). One fibre comprises of $12.7 \mathrm{mg}$ tetracycline hydrochloride and $38.1 \mathrm{mg}$ of ethylene/vinyl acetate (VA) copolymer (40\% VA). Initially, subgingival scaling and root planing was performed under local anaesthesia for all pockets (except for 1 untreated control) and fibres were placed in the study sites. The fibre was wrapped one turn loosely around the entire periphery of the tooth and pushed down to the base of the periodontal pocket by passing it through the contact points using dental floss or a plastic instrument. The remaining part of the pocket was filled with the fibre using, a back-and-forth layering technique, up to the gingival margin without creating excessive tension in the soft tissues. The length of fibre applied was recorded. Any excess fibre was cut off and discarded (Figure 2.9).

A cyanoacrylate adhesive (Octyldent, TRI-Point Medical L.P Raleigh N.C) was applied along the gingival margin, covering the top layer of the fibre and a periodontal dressing (Coe-Pak, GC America INC. Chicago, IL) was placed. The fibre was removed 10 days later. The average length of fibre required was 11.51 centimetre per tooth (range 5-23 


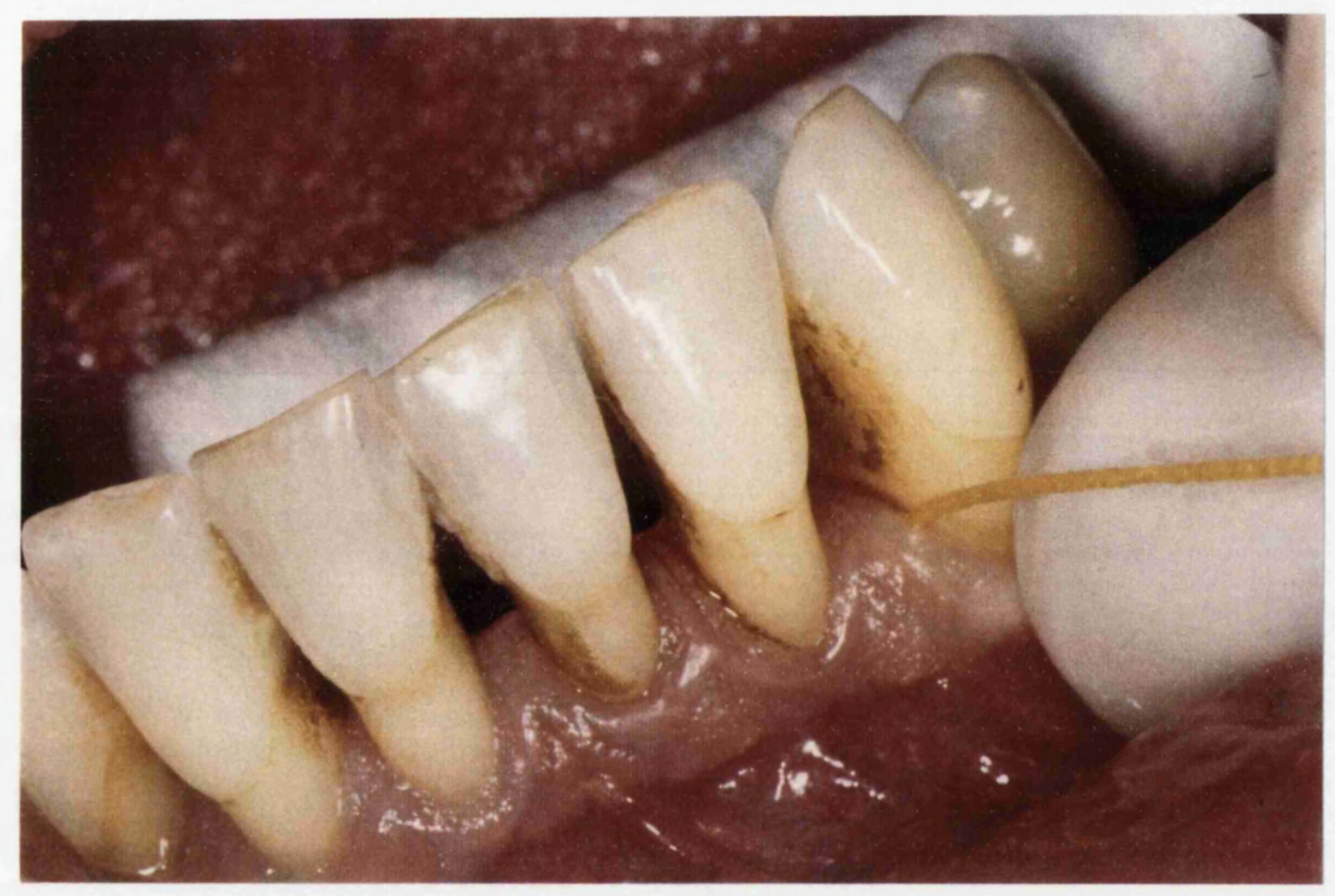

Figure 2.9 Application of tetracycline fibre 
$\mathrm{cm} /$ tooth, depending on tooth type) and the average time required to place the fibre was 8.23 minute per tooth (range 5-20 minutes, depending on tooth type).

Minocycline (Dentomycin, Cyanamid international, Lederle Division, Wayne, NJ) is a yellow ointment formulation which contains $2 \%$ minocycline hydrochloride and is applied into the pocket via a disposable applicator, once every 14 days for 3 treatments. Since the material is unstable at room temperature, the applicators were kept in a refrigerator until 20 minutes before use.

After subgingival scaling and root planing under local anaesthesia, the minocycline dental gel was applied to the pocket until it was overfilled (Figure 2.10). The excess was removed using cotton pellets. The average time required to apply the gel was less than 1 minute per tooth. This medication was repeated 14 days and 28 days after the initial application. 


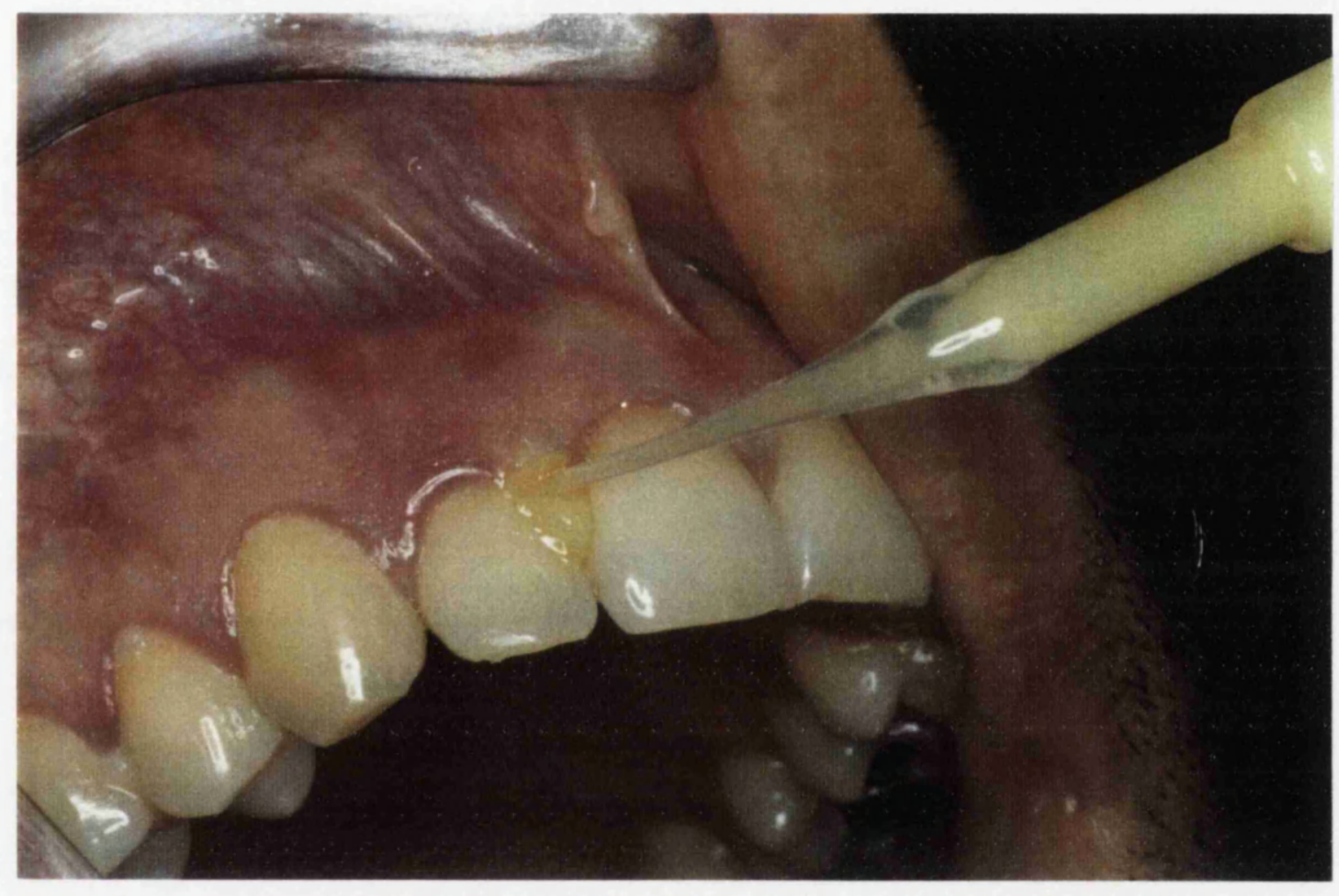

Figure 2.10 Application of minocycline gel 
Metronidazole dental gel (Elyzol, Dumex, Denmark) is a white semisolid suspension containing $25 \%$ metronidazole benzoate in a mixture of glyceryl mono-oleat (GMO) and triglyceride (sesame oil). It is dispensed in a cartridge containing $1 \mathrm{~g}$ of gel corresponding to $250 \mathrm{mg}$ metronidazole per $g$ and is injected into the pocket using an intraligamentous injection syringe with a blunt needle.

After subgingival scaling and root planing under local anaesthesia, the metronidazole dental gel was applied into the pocket until it was overfilled. The excess was removed using cotton pellets. The average time required to apply the gel was less than 1 minute per tooth. The application was repeated 7 days later.

The control group received only subgingival scaling and root planing under local anaesthesia. In an effort to standardise the scaling for the patients approximately 5 minutes scaling per tooth was allowed. 
The Whatman grade 4 paper strips (Whatman Labsales Ltd., Maidstone, Kent) were cut manually to a standard site, 13 $\mathrm{mm}$ in length and $2 \mathrm{~mm}$ wide, using a steel ruler and scalpel. A line was drawn on each strip at $8 \mathrm{~mm}$, indicating the length of paper strip to be inserted between the periotron jaws and that part of the strip to be used for GCF sampling. Rubber gloves were worn during their preparation in order to avoid contamination of the strips by substances from the operators hands. The strips were then placed in glass universal bottles for autoclaving and storage.

\subsubsection{ELISA buffers and reagents}

The buffers employed were as followings:

1) Coating buffer (CB): $1.59 \mathrm{~g} \quad \mathrm{Na}_{2} \mathrm{CO}_{3}, 2.93 \mathrm{~g} \quad \mathrm{NaHCO}_{3}$ was dissolved in $800 \mathrm{ml}$ distilled $\mathrm{H}_{2} \mathrm{O}$. The $\mathrm{pH}$ was adjusted to 9.6 at just under 1 litre, by adding $1 \mathrm{M}$ HCl up to 1 litre in a volumetric flask. It was stored in a sterilised bottle at $4^{\circ} \mathrm{C}$ for a maximum of 1 week. 


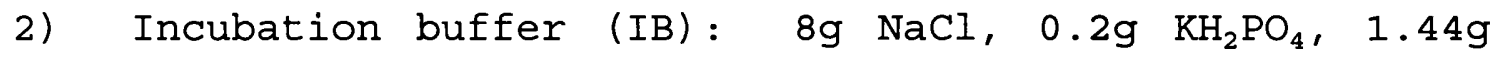
$\mathrm{Na}_{2} \mathrm{HPO}_{4} \cdot 2 \mathrm{H}_{2} \mathrm{O}, 0.2 \mathrm{~g} \mathrm{KCl}, 0.5 \mathrm{~g}$ Tween 20 (SIGMA chemical company Ltd., Poole, Dorset) dissolved in 800 millilitre of distilled $\mathrm{H}_{2} \mathrm{O}$, and made up to 1 litre with the addition of Ig of lyophilised bovine serum albumin (BSA) (SIGMA, St. Louis, USA). This layered on the surface without mixing. It was stored at $4^{\circ} \mathrm{C}$ for a maximum of 1 week (pH 7.4).

3) Wash buffer (PBST): This was prepared at 10 times the concentration of incubation buffer (nil BSA) and stored at room temperature (RT). It was diluted $1 / 10$ immediately before use.

4) Phosphate buffered saline (PBS): PBS was prepared by dissolving $8 \mathrm{~g} \mathrm{NaCl}, 0.2 \mathrm{~g} \mathrm{NaCl}, 0.2 \mathrm{~g} \mathrm{KH} \mathrm{PO}_{4}, 0.2 \mathrm{~g} \mathrm{KCl}, 1.44 \mathrm{~g}$ $\mathrm{NaH}_{2} \mathrm{PO}_{4} \cdot 2 \mathrm{H}_{2} \mathrm{O}$ up to 1 litre of distilled water ( $\mathrm{pH} 7.4$ ).

$2 \cdot 6 \cdot 3$

Antibodies and purified standards for ELISAs

The antibodies which were used in the ELISA included: Mac 78 anti-stromelysin monoclonal antibody and rabbit antistromelysin polyclonal antibody, Mac 19 and Mac 15 antiTIMP monoclonal and purified recombinant human stromelysin (SL) and also tissue inhibitor of metalloproteinases (TIMP) were donated by Cell Tech. Ltd. Slough, UK. Donkey anti 
rabbit-HRP conjugated IgG was purchased from Jackson Immuno-research Laboratories, Avondale, PA, USA.

In order to transform the Periotron digital readings for each paper strip into volumes, and also to verify the accuracy of the instrument the following procedure was carried out. First the blank periopaper was placed between the jaws of the machine and the reading dial was set to zero. Known volumes of PBS plus serum (in equivalent volume) were delivered to Whatman grade 4 paper strip with a Hamilton microsyringe at a range of volumes $(0.05-1 \mu 1)$ and Periotron readings taken. Each measurement was performed 3 times. The mean value for each volume was used in a linear regression analysis, from which the slope and intercept were used to determine the volumes of the GCF collected. The curve was analysed in two separate lines (fitted by regression method) as shown in Figure 2.11. The upper portion included volumes of 0.2 , to $1 \mu 1$ whereas the lower portion included volumes of 0.05 , to $0.2 \mu l$. The calibration was performed each day samples were taken, in 


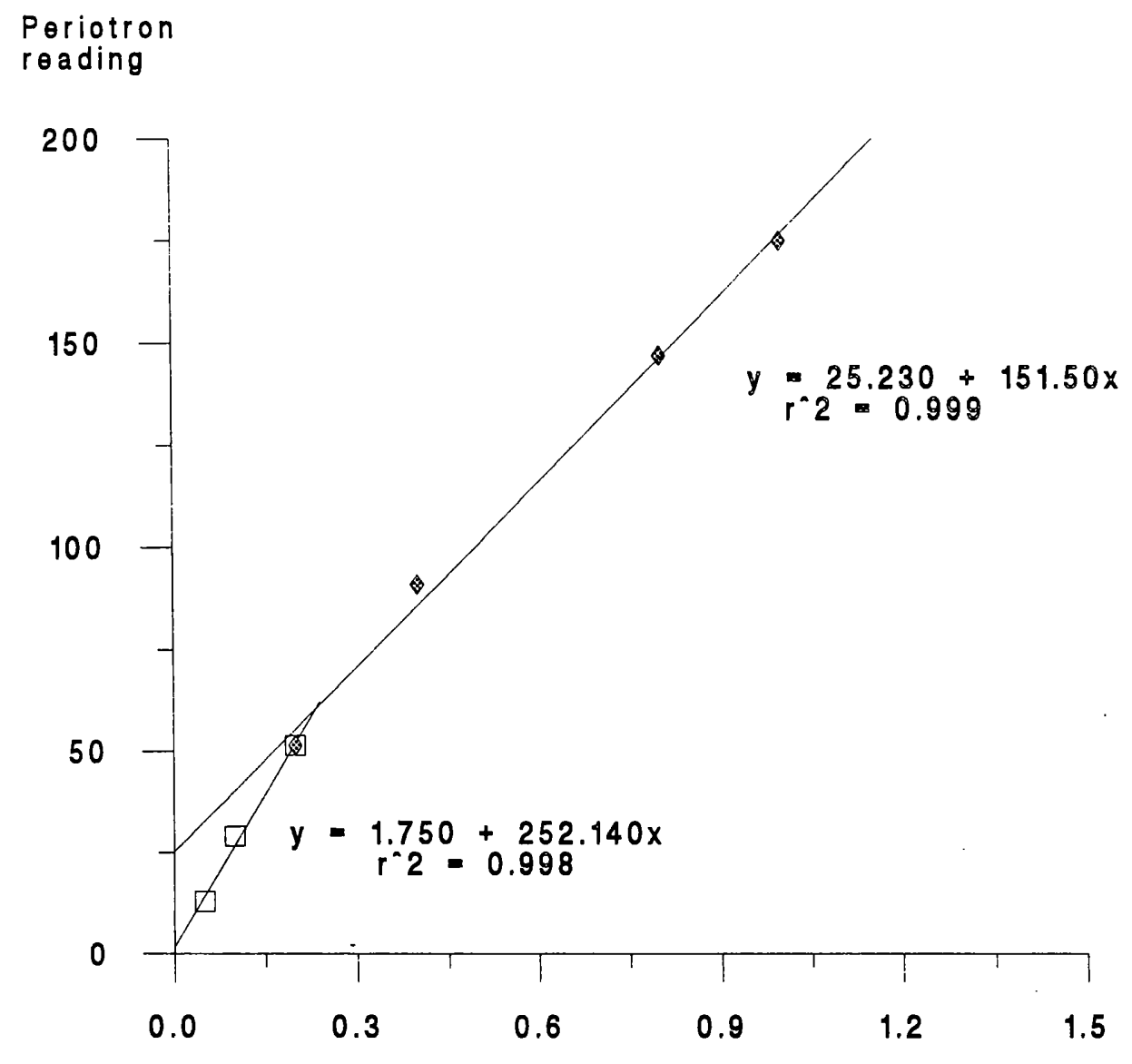

Volume (ul)

Figure 2.11 Calibration curve of the Periotron 6000 at the volume range of 0.05 to $0.2 \mu l$ and 0.2 to $1 \mu 1$. Each point represents the mean of three measurements.

$a=$ the intercept

$\mathrm{b}=$ the volume coefficient

$r=$ Pearson correlation coefficient 
order to reduce daily variability and thus improve accuracy.

\subsubsection{Gingival crevicular fluid elution}

GCF samples were eluated in $500 \mu l$ of incubation buffer for 1 hour at room temperature using a rotary mixer. The strips were then discarded and the elutes were aliquoted and stored at $-30^{\circ} \mathrm{C}$, until further procedures. Previous studies (Haerian, 1994) demonstrated that the elution of GCF samples in $500 \mu l$ of incubation buffer would yield concentrations in the eluate within the precision range of each ELISA.

\subsubsection{Sandwich ELISA methodology}

The sandwich ELISA method was a modification of the technique described previously by Cooksley et al. (1990). The modifications used were as follows: a) the sample volume in each well was reduced from $200 \mu l$ to $50 \mu l$; since we diluted the samples in $500 \mu l$ of incubation buffer and required sufficient volume for two different ELISAs and at the same time some back up volume; b) all the paper strips were eluated in the incubation buffer; since we had to use the elute from the same paper strip for different ELISAs it 


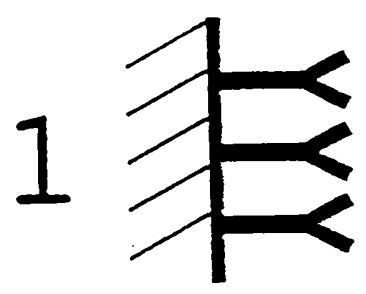

\section{Coating with first antibody}

\section{Adding standard/ sample antigen}

\author{
Incubating with \\ second antibody
}


was impossible to use two different assay buffers to elute each individual paper strip; c) the calibration line was altered toward the lower concentration, based on the range of absolute amounts of SL and TIMP detected in preliminary experiments.

This method assays the amount of antigen present in the sample as directly proportional to the amount of the second antiserum (rabbit), which was quantified indirectly by the addition of horse radish peroxidase (HRP) conjugated antirabbit IgG (donkey) (Jackson Immuno-research Laboratories, Avondale, USA) resulting in high optical densities (ODs) for high antigen concentration.

The results of experiments already carried out in our labratory (Haerian, 1994) demonstrated that among five different types of Immulon microplates (Dynatech Laboratories), the Immulon IV plates showed the least variability for the sandwich ELISAs. In summary, (Figure 2.12), the 96-well polystyrene microplate (Immulon 4 Dynatech Laboratories, Billingshurst, Sussex, U.K) was coated with the first antibody, specific to the antigen to be quantified. The eluate of the sample was then added and any antigen present was captured by immobilised antibody. The plate was then incubated with a biotin-conjugated second monoclonal antibody. Finally the plate was incubated with Extr Avidin-Peroxidase (Sigma). 
Table 2.1 Steps and reagents for stromelysin (SL) and tissue inhibitor of metalloproteinase (TIMP) sandwich ELISAs.

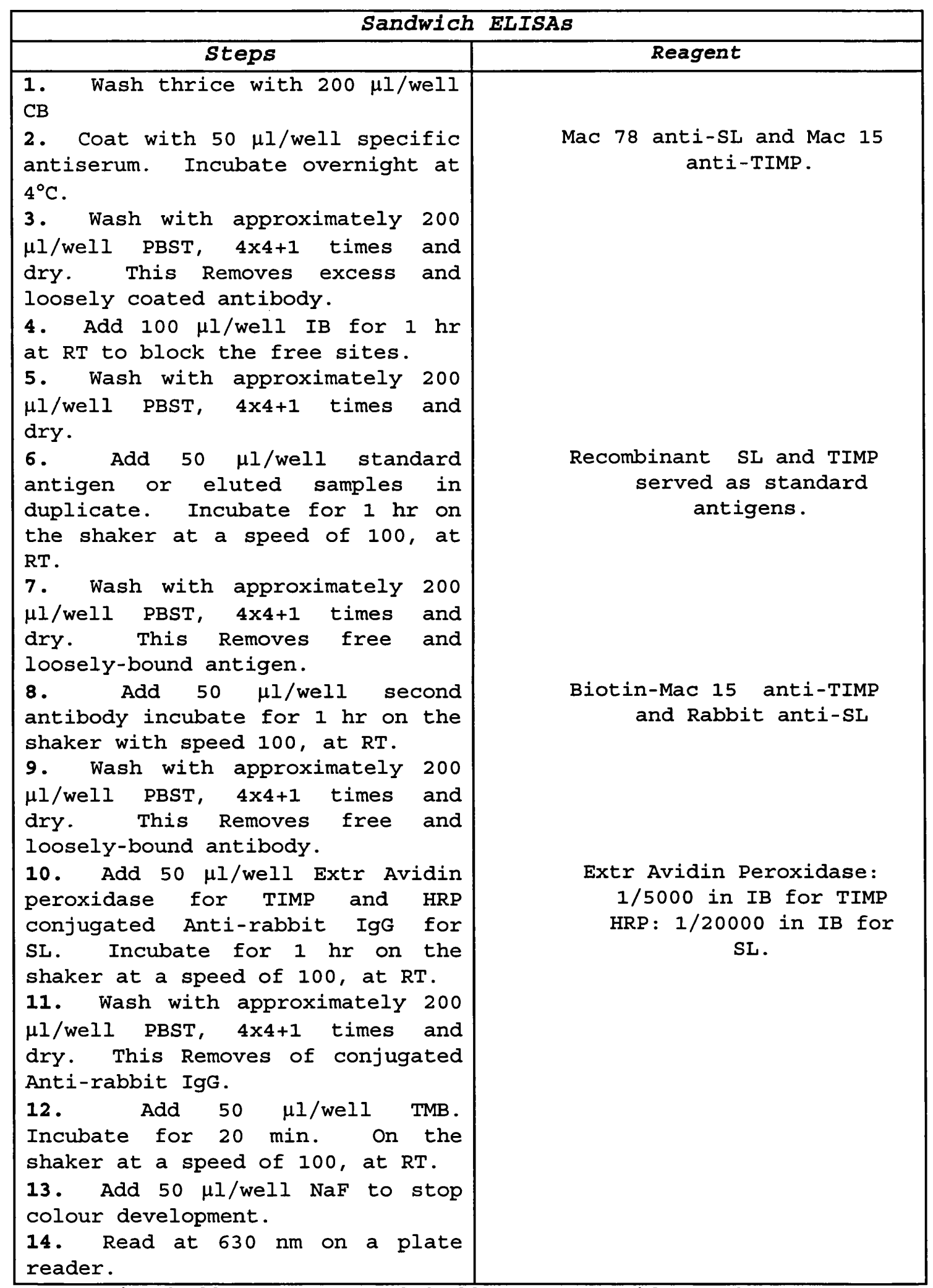

CB=Coating buffer RBST=Wash buffer TMB=Peroxidase substrate IB=Incubation buffer $\mathbf{R T}=$ Room temperature HRP=Horse Radish Peroxidase 
Visualisation was achieved by incubation with tetramethylbenzidine (TMB) substrate, and the reaction stopped with $2.5 \%$ Sodium fluoride (NaF). The plate was read at $630 \mathrm{~nm}$ on a Dynatech $\mathrm{MR5000}$ plate reader. The method is described in detail in Table 2.1. Serial dilutions of standard antigen were run for each plate. The working range of the standard antigen for the stromelysin and TIMP was 12.5-0.025 nanogram per millilitre (ng/ml) in incubation buffer (IB) and their optical densities (ODs) were used in a linear regression analysis from which the slope and intercept were used to determine the level of SL and TIMP in the samples. The precision range was defined as the linear part of the working range. The typical standard curves (precision ranges) of the SL and TIMP sandwich ELISAs were 6.25 to 0.05 (Figure 2.13). The least squares method was used to plot the best fitting line and $r^{2}>0.99$ were obtained for all assays. Linear functions of the type $\log _{n} O D=a+b x\left(\log _{n} c\right)$, where OD=Optical density, $\mathrm{c}=$ concentration, $\mathrm{a}=$ the intercept and $\mathrm{b}=$ the $\log _{n} \mathrm{c}$ coefficient were thus produced, and used for the determination of sample antigen quantities. A similar standard curve was generated and a new line fitted each time an assay was run.

The central wells were used on each plate for standards or samples in order to avoid the edge-effect (Tijssen, 1985). The peripheral wells were used for assaying the controls 
(a)

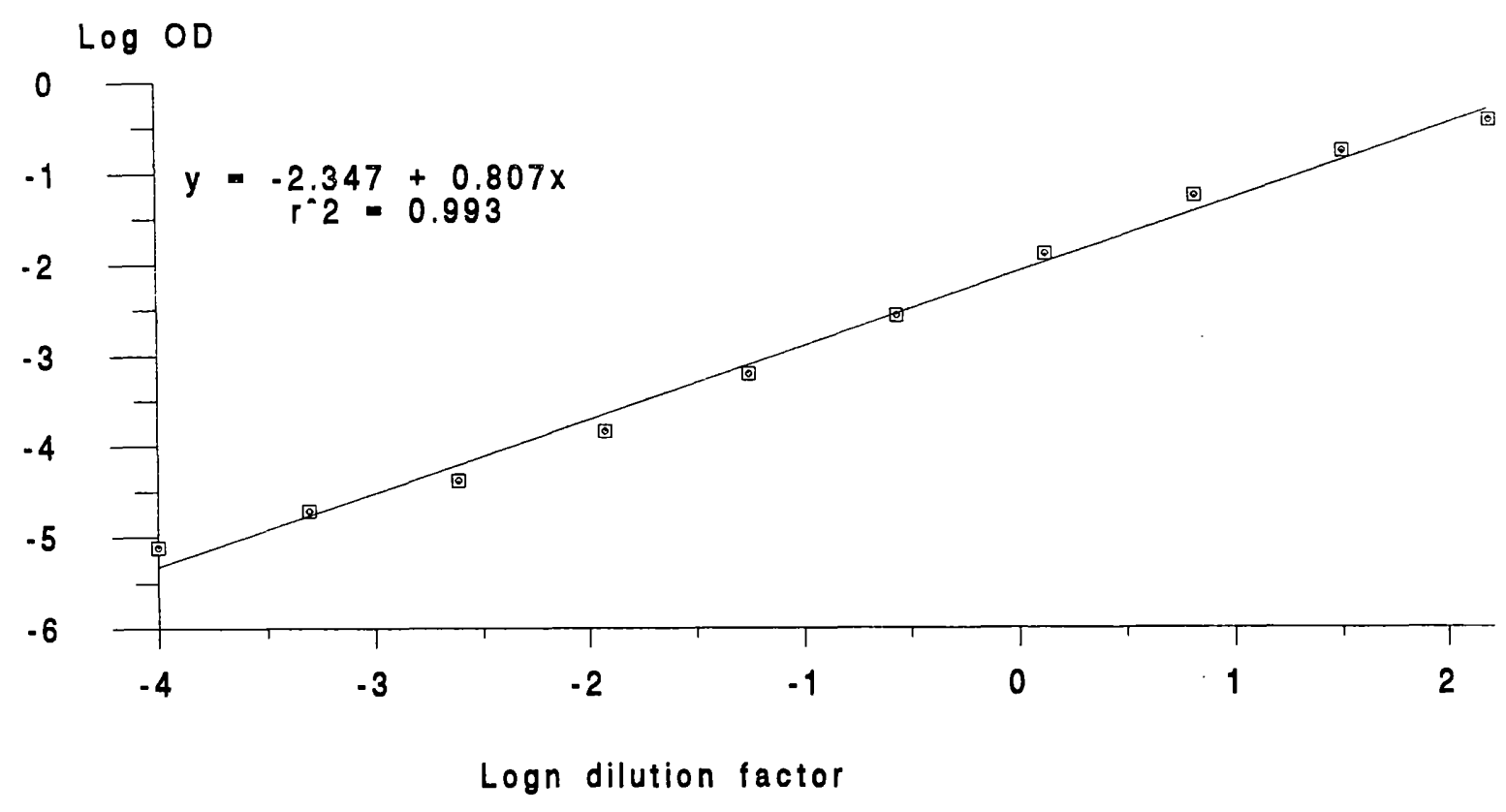

(b)

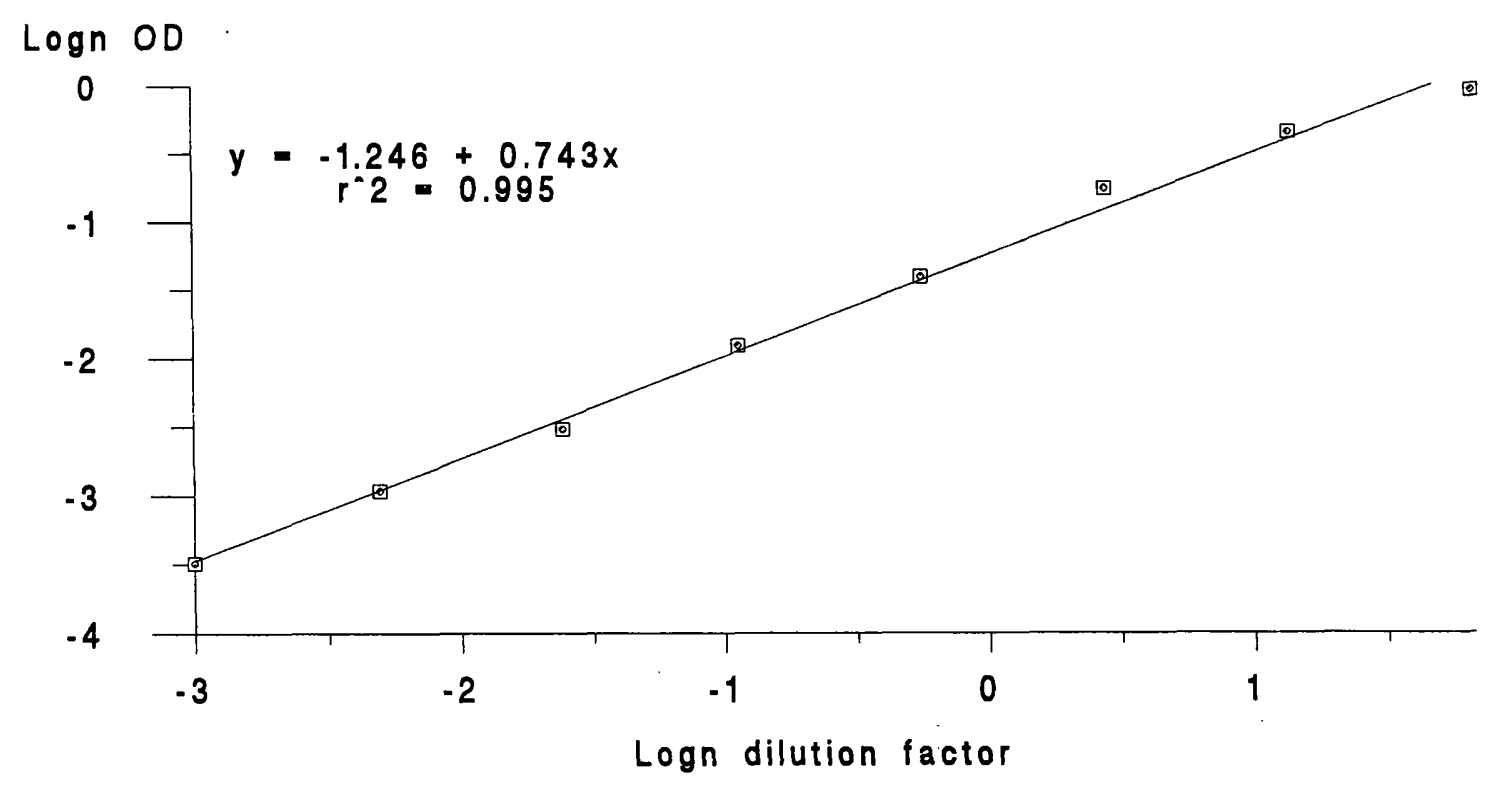

Figure 2.13 Typical calibration curve for (a) stromelysin (SI) and (b) tissue inhibitor of metalloproteinase (IIMP) 
(except for the control 14) (Table 2.2), and were run in duplicate. Zero antigen wells (Controls 14) were run in quadruplicate. Control wells (control 14, Table 2.2) in the central wells in each plate were included which contained no sample or standard antigen, in order to calculate background binding.

The mean plus two standard deviations (SD) of this background reading were taken as the cut-off point for detectability (Tijssen, 1985).

SL and TIMP quantification was performed on the eluates of GCF, and all constituents were assayed by means of sandwich enzyme linked immunosorbent assays (ELISA), based on a modification of the method of Cooksley et al. (1990). The 96-well polystyrene microplate (Immulon 4 Dynatech Laboratories, Billinghurst, Sussex, U.K) was coated with the first antibody, specific to the antigen to be quantified. These antibodies were Mac 78 anti-stromelysin monoclonal antibody for SL and Mac 19 anti-TIMP monoclonal antibody for TIMP. After overnight incubation at $4^{\circ} \mathrm{C}$, these were washed, blocked and incubated with serial dilutions of recombinant human stromelysin and TIMP. The GCF samples were then eluted in an incubation buffer 
Table 2.2 Control tests for sandwich ELISAs. Standard was added at a fifth concentration of the standard curve. When the addition of a reagent was omitted the equivalent volume of the respective buffer alone was added.

\begin{tabular}{|c|c|c|c|c|}
\hline \multicolumn{2}{|c|}{ Control } & \multicolumn{3}{|c|}{ Step } \\
\hline & $\begin{array}{c}\text { First } \\
\text { antibody } \\
\text { coating }\end{array}$ & standard & $\begin{array}{c}\text { Second } \\
\text { antibody } \\
\text { (Antiserum) }\end{array}$ & $\begin{array}{c}\text { HRP/Extr } \\
\text { Avidin } \\
\text { Peroxidase }\end{array}$ \\
\hline 1. & - & - & - & - \\
\hline 2 . & + & - & - & - \\
\hline 3 . & - & + & - & - \\
\hline 4 . & - & - & + & - \\
\hline 5 . & - & - & - & + \\
\hline 6 . & + & + & - & - \\
\hline 7 . & + & - & + & - \\
\hline 8 . & + & - & - & + \\
\hline 9 . & - & + & + & - \\
\hline 10. & - & + & - & + \\
\hline 11. & - & - & + & + \\
\hline 12 . & + & + & + & - \\
\hline 13. & + & + & - & + \\
\hline 14 . & + & - & + & + \\
\hline 15 . & - & + & + & + \\
\hline
\end{tabular}


containing $0.1 \%$ protease-free bovine serum albumin (BSA) (Calbiochem), for one hour at $25^{\circ} \mathrm{C}$ with constant mixing. The plate was then incubated with a biotin-conjugated second monoclonal antibody or HRP-conjugates rabbit-anti-SL in the case of $\mathrm{SL}$ in the same buffer for one hour at $25^{\circ} \mathrm{C}$ with constant mixing. Biotinylated Mac 15 antibody was used to reveal TIMP. The plate was washed and incubated with HRP-anti-rabbit IgG (Jackson) in the stromelysin assay and Extr Avidin-peroxidase (Sigma) in the TIMP assay in the same buffer for one hour at $25^{\circ} \mathrm{C}$ with constant mixing.

Visualisation was achieved by incubation with $3,3^{\prime}, 5,5^{\prime}-$ tetramethylbenzidine (TMB) (KPL) for 15 minutes at $25^{\circ} \mathrm{C}$ with constant mixing and the reaction stopped with $2.5 \% \mathrm{NaF}$ (Sodium fluoride). The plate was read at $630 \mathrm{~nm}$ (dual wavelength with 490 nanometre as reference wavelength), on a Dynatech MR 5000 plate reader. Serial dilutions of standard were run for each individual plate and their oDs used in a linear regression analysis from which the slope and intercept were used to determine the sample concentration of SL and TIMP (Figure 2.13).

Results were expressed as picogram per 30 second (pg/30s) sample. Two wells in each plate had no sample or standard antigen so as to calculate background reading. The mean plus two standard deviations of this background reading was 
taken as the cut-off point for detectability (Tijssen, 1985).

2.8 Preliminary experiments

The results of experiments already carried out in our laboratory (Haerian, 1994) demonstrated that:

a) Among five different types of immulon (Dynatech Laboratories) microelisa plates, the immulon plates were selected for the sandwich ELISAs in these studies.

b) There was no significant difference between the levels of GCF components on different plates at the same concentration and the intra-plate variability was low for levels of SL and TIMP GCF.

c) Serum components did not interfere in the assaying of GCF SL and TIMP.

d) GCF SL and TIMP respond in a similar way to the highly purified standard and it was concluded that the assays developed could be used for their quantification in GCF. 
e) The elution of GCF samples in $500 \mu 1$ of incubation buffer would yield concentrations in the eluate within the precision range of each ELISA.

f) The SL and TIMP sandwich ELISAs described by Cooksley et al. (1990) were modified.

$2.8 \cdot 1$

The reproducibility of stromelysin (SL) and tissue inhibitor of metalloproteinase (TIMP) on gingival crevicular fluid paper strip samples

This part of the study was designed to determine the reproducibility of stromelysin (SL) and tissue inhibitor of metalloproteinase (TIMP) sampled from gingival crevicular fluid (GCF), and recovered on filter paper strips. A total of 310 sites from 52 patients were sampled in duplicate. The first and second samples were taken with a 1 minute interval between. The sampling of the same sites was repeated after treatment, resulting in a total of 620 pairs of samples (1240 samples).

The GCF SL was detectable in 180 (38\%) out of 620 sites in the first set of samples, and 150 (24\%) out of 620 sites in the second set of samples. The mean level of GCF SL was higher in the first samples than the second samples. 
However, the paired t-test failed to demonstrate a significant difference when the first and second samples were compared for their GCF SL levels $(p=0.18)$ (Table 3.3).

TIMP was detected in 615 (99\%) of the first samples, and $597(96.2 \%)$ out of 620 sites of the second samples. The mean level of GCF TIMP decreased significantly in the second sample compared to the first sample. The paired ttest demonstrated a significant difference when the first and second samples were compared for their GCF TIMP levels $(p<0.001) \quad($ Table 3.3) .

Correlation between the first and second samples was 0.22 $(p<0.001)$ and $0.20 \quad(p<0.001)$ for the SL and TIMP levels respectively. The correlation between the GCF volumes of the first and second samples was $0.62(\mathrm{p}<0.001)$.

\section{Methodological considerations}

Several investigators have shown the first sample to be the richest in the components to be measured (Lamster et al., 1989; Persson \& Page 1990). Other investigators have discarded the first sample, or used it to measure volume, and used subsequent samples for analysis (Binder et al., 1987; Larivée et al., 1986). Binder et al. (1987) harvested repeated samples of GCF and measured fluid volume and total content and concentration of alkaline and acid 
Table 2.3 Comparison of Mean (SEM) GCF level of SL, TIMP and volume in the first sample and second sample $(n=620)$, using Paired t-test.

\begin{tabular}{|c|c|c|c|}
\hline & GCF SL & GCF TIMP & GCF volume \\
\hline First samples & $10.37(1.23)$ & $359.9(16.1)$ & $0.432(0.01)$ \\
\hline Second samples & $8.77(0.75)$ & $138.79(7.13)$ & $0.272(0.01)$ \\
\hline p value & 0.18 & $<0.001$ & $<0.001$ \\
\hline
\end{tabular}


phosphatase activities. They concluded that the first sample harvested should be discarded and analysis performed on second or later replicate samples. Our observations do not confirm the model of GCF flow and composition suggested by Binder et al. The differences in our observations and those of Binder et al. (1987) may result from differences in sampling technique or in the enzymes measured. GCF contains many enzymes of differing molecular size and concentration; one might expect the kinetics of accumulation of enzymes on filter-paper strips, to differ one from another, and also we measured the amount of antigenic enzyme whereas they measured the activity of enzyme.

The fluid component of GCF arises from the vessels of the microcirculation of the gingiva, and traverses the inflamed tissue during which it picks up enzymes being released from diseased tissue; the enzyme containing fluid enters the reservoir in the pocket or sulcus where enzymes become more concentrated. It is not supposed that over a short time period of seconds or minutes, the rate of production of stromelysin from the fibroblast cells, the rate of release of stromelysin from the tissues, the rate of passage of fluid from blood vessels to the fluid reservoir and the exit of fluid from the reservoir, to be in a steady-state of equilibrium. The first sample may deplete entirely or in part, the enzyme-rich reservoir during the 30 seconds. 
The fluid that replenishes the reservoir is less enzymerich, and replicate sampling, especially with short time intervals, may result in a washout of enzyme from the disease-active tissue, at least in the short term, when samples are taken at a 1 minute intervals.

It is concluded that the first sample should be preferred to the second GCF sample. This may be particularly important for the assessment of GCF TIMP.

The data were analysed using the Minitab version 9.2 and the SPSS/PC ${ }^{+}$statistical packages. The study of GCF stromelysin and tissue inhibitor of metalloproteinases in gingival crevicular fluid was carried out to evaluate the changes of the GCF levels of SL and TIMP after treatment with three different locally delivered antibiotics plus scaling and root planing or scaling and root planing alone as a control treatment. Furthermore, the study was planned to assess the ability of GCF SL and TIMP to predict further response to the treatment. First, the distribution of the data was inspected using histograms, and it was found that the data had a markedly skewed distribution. While, log transformation $\left(\log _{10}(1+x), x=o r i g i n a l\right.$ data value) was not successful in producing a normal distribution in SL data, 
it was successful in TIMP data. Therefore, parametric tests were utilised for TIMP and non parametric tests were performed for SL.

The comparisons were made between sites with bleeding on probing (BOP) versus (vs.) sites without BOP, between suppuration sites vs. sites without suppuration. Moreover, the diseased sites were broken down according to the smoking status, gender, age and site position (anterior vs. posterior sites) and the two sample comparisons, were made using the Mann-Whitney $U$ test for SL and the two sample $t-$ test for TIMP. Comparison of sites with different MGI was made using the Kruskal-Wallis test. The Spearman rank correlation coefficients were calculated to examine the relationship between biochemical parameters and pocket depth or GCF volume. Discriminant analysis was performed to disclose the clinical characteristics of sites with different levels of GCF SL or TIMP.

Analysis of the clinical findings was performed using both site and patient as the experimental unit. When the subject was considered as the unit, sites within each subject were averaged and one score produced per subject for each clinical and biochemical parameter. The mean detection limit of the ELISA assay was divided by two and was used as the SL and TIMP levels for the sites at which these biochemical components were not detected. 
The principal criteria of response to the treatment were changes in level of GCF SL and TIMP, in this study. The mean differences were utilised for the analysis of SL and TIMP in terms of response to the treatments. Histogram showed normal distribution after log transformation, therefore, parametric analysis were performed. The analysis of the effect of treatment on the biochemical and clinical parameters was performed as follows; For each treatment group the changes in the GCF SL and TIMP and clinical parameters were calculated and subjected to the paired t-test. Analysis of variance (ANOVA) was employed on the log transformed data in order to determine significant differences in the changes in GCF levels of SL and TIMP between different treatments. When a significant effect was detected by the use of ANOVA, follow up analysis was performed using Tukey's test in order to determine the direction and location of the differences. The analysis of covariance (ANCOVA) was used with the baseline SL or TIMP levels as continuous covariantes. By using the ANCOVA, the variability among the sites and treatment groups which existed at the baseline levels of GCF SL and TIMP was taken into account mathematically. If a significant difference was found between treatments, then the post-hoc comparisons were performed by comparing only two groups at a time. The probability threshold for statistical significance was set at $0.020(0.05 / \sqrt{ } 6$, where 6 number of comparisons, Brown $\&$ Swanson Back, 1988). 
The effects on GCF SL and TIMP changes of factors such as smoking status, gender, age and site position were analysed using the two sample t-test. The correlation between the post-treatment levels of biochemical and clinical parameters were examined by the non-parametric Spearman rank correlation coefficients.

The General Linear Model (GLM) procedure, which performed analysis of variance for unbalanced data, was used to find out if there were statistically significant differences in the changes of stromelysin levels, taking into account the effect of other potentially important factor such as pocket depth, smoking status, the location of site, age, and sex.

In order to examine the ability of the GCF SL and TIMP baseline levels to predict the treatment outcome, (regardless of treatment type) two extreme categories of sites were selected as the best responding sites (BRS) and the worst responding sites (WRS). The criteria for selection were; WRS: $\mathrm{PD}>3.5$ plus $\mathrm{BOP}$ at the reassessment, BRS: $P D \leq 3.5$ and no $B O P$ at the reassessment visit. The WRS and BRS categories were than compared using the MannWhitney U test. Moreover, the difference between WRS and BRS on changes in their biochemical profile and GCF volume ( $\Delta \mathrm{SL}, \Delta \mathrm{TIMP}$ and $\Delta \mathrm{Vol}$ ) were accomplished using the two sample $t$-test on the $\log _{10}$ transformed data. Finally, a similar analysis was performed on another two extreme 
categories. The criteria for these categories were: a) sites which showed attachment gain>1 $\mathrm{mm}$ b) sites which showed attachment loss $(\Delta A L<0)$.

In the study of repeated sampling of stromelysin (SL) and tissue inhibitor of metalloproteinases (TIMP) from gingival crevicular fluid, SL and TIMP were assessed in the same samples and results were expressed as absolute amounts i.e. pg/30s of sample collection. The differences in the stromelysin levels of each site between the first and the second sample were subjected to paired t-test. 
CHAPTER 3

\section{RESULTS}


A cross-sectional analysis of the association between GCF SL and TIMP levels and clinical parameters

A total of two hundred and six sites with recurrent periodontitis from 52 patients were sampled. Two diseased sites were excluded for technical reasons, one site lost its tetracycline fibre before the 10 day placement period finished and in the scaling group a further site could not measured. Within each patient, 4 diseased sites were selected. The logarithmic transformation failed to produce a normal distribution of data for SL, but was successful with TIMP at the baseline. Therefore, non-parametric tests (Mann-Whitney $U$ test) for $S L$ and parametric tests (two sample t-test) for TIMP were performed at the baseline. The relationship of biochemical parameters with pocket depth and GCF volume were examined with spearman rank correlation. The relationship of biochemical parameters with pocket depth and GCF volume were performed with Spearman rank correlation. 
Comparison of GCF levels of stromelysin and TIMP between bleeding and non-bleeding sites, suppurating and non-suppurating sites and sites with different levels of gingival inflammation at the baseline

SL was detectable in only 61 (29.6\%) out of 206 sites. The level of SL was higher in sites with bleeding on probing $(n=185)$, than sites which did not bleed on probing $(n=21)$, however the difference was not statistically significant $(p=0.47)$ (Table 3.1). The level of SL was lower in sites with suppuration $(n=27)$, than sites without suppuration $(n=179)$ but the difference was not significant $(p=0.36)$ (Table 3.2). The level of SL increased with increasing gingival inflammation scores and the Kruskal-Wallis test showed this difference was significant $(p=0.028)$ (Table $3.3)$.

TIMP was detected in $203(98.5 \%)$ sites. The TIMP level was lower in bleeding sites versus non-bleeding sites, however it was not statistically significant (Mann-Whitney U test $\mathrm{p}=0.62$ ) (Table 3.1). The TIMP level was higher in suppurative sites $(n=27)$ compared to the sites which did not show suppuration $(n=179)$, but this difference was not significant $(p=0.12) \quad(T a b l e ~ 3.2)$. No relationship was observed between different gingival inflammation scores and GCF TIMP level $(p=0.395)$ (Table 3.3). 
Table 3.1 Comparison of GCF SL and TIMP levels in sites with bleeding on probing ( $B O P$ ) and sites without bleeding on probing at the baseline, using Mann-Whitney $U$ test for SL and two sample $t$-test for TIMP $(n=206)$. Mean \& (SEM) are given.

\begin{tabular}{l|cccc}
\hline & $\mathbf{n}$ & Stromelysin(pg/30s) & TIMP (pg/30s) \\
\hline Negative BOP & 21 & 18.57 & $(5.12)$ & $322(110)$ \\
Positive BOP & 185 & $20.59(2.47)$ & $291.2 \quad(25.2)$ \\
\hline p value & & 0.47 & 0.62 \\
\hline
\end{tabular}

Table 3.2 Comparison of GCF SL and TIMP levels in sites with suppuration and sites without suppuration at the baseline, using Mann-Whitney $U$ test for SL and Two sample $t$-test for TIMP $(n=206)$. Mean \& (SEM) are given.

\begin{tabular}{|c|c|c|c|}
\hline & $\overline{\mathbf{n}}$ & stromelysin (pg/30s) & TIMP (pg/30s) \\
\hline $\begin{array}{l}\text { Negative } \\
\text { Suppuration }\end{array}$ & 179 & $21.09(2.59)$ & $281.1(26.8)$ \\
\hline $\begin{array}{l}\text { Positive } \\
\text { Suppuration }\end{array}$ & 27 & $15.69(2.60)$ & $381.9(71.1)$ \\
\hline p value & & 0.36 & 0.12 \\
\hline
\end{tabular}


Correlation between probing depth, GCF volume and GCF levels of matrix metalloproteinases and their inhibitor

The correlation of SL and TIMP with the probing depth and GCF volume is shown in Table 3.4. Overall, SL and TIMP's correlation with pocket depth and GCF volume were either weak or not significant.

$3 \cdot 1 \cdot 3$

Comparison between GCF level of SL, TIMP,
and GCF volume, considering smoking,
gender, age and anterior vs.
posteriorsites

The effect of smoking on the level of GCF SL, TIMP and volume in diseased sites were compared. Results showed that the level of GCF SL, and GCF volume were lower in smokers than non-smokers in the diseased sites whereas the mean level of TIMP was higher in these sites in smokers than non smokers. However, none of the differences were significant ( $p$ value for $S L=0.22$, TIMP=0.34, Vol=0.39) (Table 3.5).

The effects of sex, age, and site on GCF SL, TIMP and volume in 206 periodontitis sites were evaluated using Mann-Whitney $U$ test for SL and two sample $t$-test for TIMP 
Table 3.3 Comparison of GCF SL and TIMP levels for different gingival inflammation scores at baseline, using Kruskal-wallis test for SL and ANOVA test for TIMP $(n=206)$. Mean \& (SEM) are given.

\begin{tabular}{|c|c|c|c|}
\hline & $\bar{n}$ & stromelysin (pg/30s) & TIMP (pg/30s) \\
\hline MGI $=0$ & 6 & $16.70(4.12)$ & $296(115)$ \\
\hline MGI $=1$ & 29 & $11.50 \quad(1.03)$ & $234.0 \quad(53.2)$ \\
\hline MGI $=2$ & 112 & $18.64 \quad(2.28)$ & $326.1(38.9)$ \\
\hline MGI $=3$ & 59 & $28.45 \quad(6.53)$ & $263.4(38.1)$ \\
\hline$p$ value & & 0.028 & 0.395 \\
\hline
\end{tabular}

MGI= Modified gingival index

Table 3.4 Spearman rank correlation coefficients between pocket depth and GCF volume and biochemical parameters (pg/30s) in GCF from 206 sites in 52 patients at the baseline examination. Probability levels ( $p$ ) are shown.

\begin{tabular}{l|cccc}
\hline & \multicolumn{2}{|c}{ Stromelysin } & TIMP \\
\hline Vol & $r=0.331$ & $(0.005)$ & $r=0.360$ & $(\mathrm{p}<0.001)$ \\
PD & $r=0.033$ & $(0.667)$ & $r=0.034$ & $(\mathrm{p}=0.678)$ \\
\hline
\end{tabular}


and volume. The mean level of the SL was higher in males $(p=0.464)$, and the anterior sites $(p=0.872)$ (Table 3.5), however, none of the differences were statistically significant. The mean GCF SL was almost identical in subjects older than 35 years $(p=0.89)$.

The mean level of TIMP was higher in males compared to females $(p=0.1)$ and was higher in posterior sites than anterior sites $(p=0.22)$. The subjects with less than 35 years showed a higher level of TIMP compared to subjects with age higher than 35 years although these differences were not significant (Table 3.5 ).

The mean GCF volume was lower in smokers than non smokers, although it was not statistically significant $(p=0.390)$. The mean GCF volume was almost identical in subjects with age more than 35 compared to subjects with age less than 35. However, the mean GCF volume was higher in males than females $(p=0.073)$ and was significantly higher in posterior compared to anterior sites $(p=0.016)$.

In order to disclose the clinical characteristic of sites with different level of SL or TIMP, discriminate analysis was performed. The grouping criteria for SL was the detectability by the ELISA. For the TIMP, the test was considered positive when a site demonstrated TIMP $\leq 370.4$ pg/30s. This threshold value represents the mean TIMP 
Table 3.5 The effects of smoking, sex, site and age on biochemical and clinical parameters in baseline, site was used as the unit of analysis $(n=206)$, means \& (SEM) are given. (Two sample $t$-test for log transform mean TIMP, PD, Vol and Mann-Whitney U test for SL, MGI, PI were used).

\begin{tabular}{|c|c|c|c|c|c|c|c|c|}
\hline & \multicolumn{2}{|c|}{ smoking } & \multicolumn{2}{|c|}{$\operatorname{sex}$} & \multicolumn{2}{|c|}{ age } & \multicolumn{2}{|c|}{ site } \\
\hline & $S m$ & non $\mathrm{Sm}$ & female & male & $<35$ & $>35$ & Ant & Post \\
\hline & $\mathrm{n}=115$ & $n=91$ & $n=128$ & $n=78$ & $n=28$ & $n=178$ & $n=145$ & $n=61$ \\
\hline \multirow[t]{2}{*}{$S L$} & 18.18 & 23.17 & 17.55 & 25.04 & 14.72 & 21.28 & 22.14 & 16.21 \\
\hline & $(1.98)$ & $(4.50)$ & $(1.88)$ & $(5.13)$ & $(1.55)$ & $(2.62)$ & $(3.17)$ & $(1.44)$ \\
\hline$p$ value & & 0.22 & & 0.46 & & 0.89 & & 0.87 \\
\hline \multirow[t]{2}{*}{ TIMP } & 315.5 & 267.5 & 253.5 & 361.3 & 313 & 291.4 & 247.8 & 404.7 \\
\hline & $(34.9)$ & $(36.2)$ & $(28.0)$ & $(47.3)$ & (102) & $(24.5)$ & $(22.9)$ & $(63.5)$ \\
\hline$p$ value & & 0.34 & & 0.10 & & 0.26 & & 0.22 \\
\hline \multirow[t]{2}{*}{ GCF VOI } & 0.442 & 0.482 & 0.427 & 0.514 & 0.431 & 0.464 & 0.422 & 0.549 \\
\hline & $(0.03)$ & $(0.03)$ & $(0.03)$ & $(0.03)$ & $(0.05)$ & $(0.03)$ & $(0.03)$ & $(0.04)$ \\
\hline p value & & 0.39 & & 0.07 & & 0.56 & & 0.02 \\
\hline \multirow[t]{2}{*}{$P D$} & 5.53 & 5.53 & 5.52 & 5.54 & 5.30 & 5.57 & 5.62 & 5.32 \\
\hline & $(0.14)$ & $(0.12)$ & $(0.11)$ & $(0.16)$ & $(0.22)$ & $(0.10)$ & $(0.11)$ & $(0.16)$ \\
\hline p value & & 0.98 & & 0.94 & & 0.26 & & 0.12 \\
\hline \multirow[t]{2}{*}{ GI } & 2.14 & 2.04 & 2.0 & 2.23 & 2.14 & 2.08 & 2.15 & 1.95 \\
\hline & $(0.08)$ & $(0.07)$ & $(0.07)$ & $(0.08)$ & $(0.13)$ & $(0.66)$ & $(0.06)$ & $(0.10)$ \\
\hline$p$ value & & 0.35 & & 0.06 & & 0.76 & & 0.14 \\
\hline \multirow[t]{2}{*}{$P I$} & 0.66 & 0.55 & 0.58 & 0.63 & 0.61 & 0.60 & 0.61 & 0.58 \\
\hline & $(0.08)$ & $(0.06)$ & $(0.06)$ & $(0.08)$ & $(0.12)$ & $(0.05)$ & $(0.06)$ & $(0.09)$ \\
\hline$p$ value & & 0.37 & & 0.74 & & 0.79 & & 0.75 \\
\hline
\end{tabular}


level in successfully treated sites at the reassessment visit.

Table 3.6, demonstrates the characteristic values for sites with detectable and undetectable SL, as well as sites with high $(>370.4 \mathrm{pg} / 30 \mathrm{~s})$ and low $(\leq 370.4 \mathrm{pg} / 30 \mathrm{~s})$ TIMP levels.

The analysis utilised a step-wise selection. To arrive at a good model, a variety of potentially useful variables are included in the data set. In the step-wise method the first variable included in the analysis has the smallest Wilk's lambda provided that its $p$ values is significant. Wilk's lambda is the ratio of the within group sum of squares to total sum of squares. After the first variable is entered, the values of the criterion are re-evaluated for all variables not in the model, and the variable with the smallest Wilk's lambda value and a significant $p$ value is entered next.

Table 3.7 shows the discriminate variables which were eligible for entering into the model. These include GCF volume and GCF TIMP level for discriminating between detectable and undetectable SL levels and GCF volume, GCF SL level and gender for discriminating between high and low TIMP levels. No other parameters had a small enough Wilk's lambda for entry. 
Table 3.6 The group mean of GCF TIMP, BOP, PI, MGI, Vol, PD, gender, Smoking, site, and age on SL and TIMP. The grouping criteria for SL was the detectability of SL by the ELISA and TIMP $\leq 370.4=0$ and TIMP $>370.4=1$.

\begin{tabular}{|c|c|c|c|c|}
\hline & & SL & & IMP \\
\hline variables & undetected & detected & $\begin{array}{l}\leq 370.4 \\
\mathrm{pg} / 30 \mathrm{~s}\end{array}$ & $\begin{array}{l}>370.4 \\
\mathrm{pg} / 30 \mathrm{~s}\end{array}$ \\
\hline Site (\% Anterior) & $71 \%$ & $67 \%$ & $75 \%$ & $54 \%$ \\
\hline BOP ( $\%$ positive) & $88 \%$ & $93 \%$ & $90 \%$ & $89 \%$ \\
\hline SUP ( $\%$ positive) & $15 \%$ & $8 \%$ & $10 \%$ & $22 \%$ \\
\hline Smoking (\%smoker) & $60 \%$ & $46 \%$ & $55 \%$ & $59 \%$ \\
\hline SEX ( $\%$ female) & $63 \%$ & $59 \%$ & $67 \%$ & $46 \%$ \\
\hline $\mathrm{PD}(\mathrm{mm})$ & 5.57 & 5.42 & 5.5 & 5.6 \\
\hline vol $(\mu 1)$ & 0.39 & 0.62 & 0.40 & 0.67 \\
\hline PI & 0.59 & 0.62 & 0.60 & 0.59 \\
\hline MGI & 2.03 & 2.21 & 2.09 & 2.07 \\
\hline Age (year) & 44.48 & 43.92 & 39.41 & 44.43 \\
\hline TIMP (pg/30s) & 309.6 & 258.0 & - & - \\
\hline sL (pg/30s) & - & - & 21.14 & 17.76 \\
\hline
\end{tabular}


Table 3.7 The Wilks' lambda and significance level of the entry action of variables in the SL and TIMP.

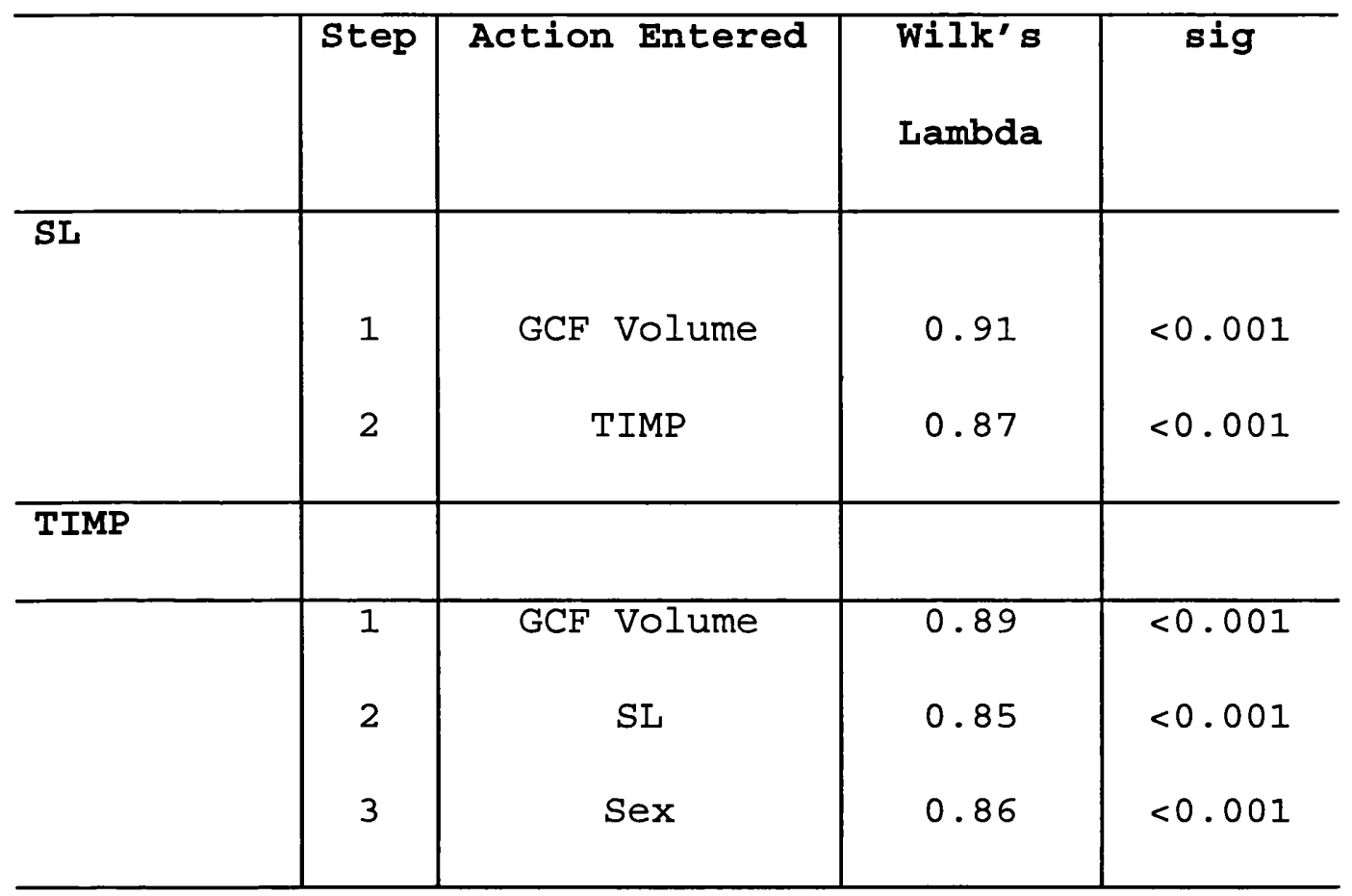


Table 3.8 and Table 3.9 show the number of cases classified correctly and incorrectly. The percent of 'grouped'" cases correctly classified was $69.42 \%$ for SL and was $70.39 \%$ for TIMP respectively.

3.2 The efficacy of adjunctive locally delivered
antibiotics on biochemical and clinical
parameters

This study looked at changes in GCF SL and TIMP as a means of monitoring clinical changes in a 4 treatment study. The pocket depth and clinical indices are reported elsewhere and was not my personal work submitted by me as part of this MSc thesis. A summary of these results however is given. Comparison of changes in pocket depth, attachment level and GCF volume between treatment groups were performed with the ANCOVA test. Comparison of changes in these parameters before and after treatment was performed using the paired t-test. Comparison of MGI and PI before and after treatments was performed, using the Wilcoxon's test and comparison between treatment groups was carried out with the Kruskal-Wallis test. Changes in BOP were assessed using the chi-square test $\left(\chi^{2}\right)$.

A total of 52 sites in 13 patients were selected in each treatment group. Six weeks after the last treatment visit 
Table 3.8 The number and percentage of cases classified correctly and misclassified by the detectability of SL.

\begin{tabular}{l|lccc}
\hline & Actual group & $\begin{array}{c}\text { No. of } \\
\text { Cases }\end{array}$ & \multicolumn{2}{c}{$\begin{array}{c}\text { Predicted group } \\
\text { membership }\end{array}$} \\
\hline & & & 0 & 1 \\
\hline Group 0 & undetectable & 145 & $\begin{array}{c}104 \\
(71.7 \%)\end{array}$ & $\begin{array}{c}41 \\
\text { Group 1 }\end{array}$ \\
& detectable & 61 & $28.3 \%)$ \\
& & & $(36.1 \%)$ & 39 \\
& & & & $(63.9 \%)$ \\
\hline
\end{tabular}

Sensitivity $39 / 61=64 \frac{1}{8}$

Specificity $104 / 145=71 \%$

Table 3.9 The number and percentage of cases classified correctly and misclassified by the dichotomous grouping of TIMP.

\begin{tabular}{l|cccc}
\hline & Actual group & $\begin{array}{c}\text { No. of } \\
\text { Cases }\end{array}$ & \multicolumn{2}{c}{$\begin{array}{c}\text { Predicted group } \\
\text { membership }\end{array}$} \\
\hline & & & 0 & 1 \\
\hline Group 0 & TIMP $\leq 370.4$ & 160 & $\begin{array}{c}117 \\
(73.1 \%)\end{array}$ & $\begin{array}{c}43 \\
\text { Group } 1\end{array}$ \\
& TIMP $>370.4$ & 76 & $18 \%)$ & 28 \\
& & & $(39.1 \%)$ & $(60.9 \%)$ \\
\hline & Total classified correctly $=70.39 \%$
\end{tabular}


all the patients were assessed at a reassessment visit. Overall, two treated sites had to be excluded from the study. The same clinical and biochemical sampling as the baseline visit were repeated at this visit. Wherever data was not normally distributed, it was logarithmically transformed in order to use parametric tests. Analysis was performed with regard to both the site and subject as the experimental unit. When the subject was considered as the unit of assessment, sites within each subject were averaged and one score produced per subject for each clinical and biochemical parameter. For sites giving values for SL and TIMP below the assay detection level, a value which was half the mean detection limit of the ELISA was used.

$3 \cdot 2 \cdot 1$

Change in the clinical finding after adjunctive treatment with different locally delivered antibiotics or scaling and root planing alone

Pocket depth: At the time of initial subgingival therapy, the average probing pocket depth was $5.5 \mathrm{~mm}$, with no significant differences between groups ( $p=0.881)$. significant testing revealed that a highly significant reduction in pocket depth occurred in all treatment groups (Table 3.10). Analysis of covariance (ANCOVA) showed significant difference between treatment groups when data 
was analysed on a subject basis $(p=0.011)$. The results indicated that only tetracycline fibre treatment resulted in a significantly higher change in pocket depth than the scaling alone group $(p=0.003)$ (Table 3.10).

Modified Gingival Index: Wilcoxon test showed that the modified gingival index was significantly decreased after the treatments in all treatment groups. There was significant difference between treatment groups in terms of changes of modified gingival index (Kruskal-Wallis test, $\mathrm{p}<0.001$ ). Mann-Whitney $U$ test demonstrated that these differences were between i) tetracycline fibre plus scaling group and minocycline gel plus scaling group ( $p=0.002)$, ii) tetracycline fibre plus scaling group and metronidazole gel plus scaling group $(\mathrm{p}=0.007)$, and $i i i)$ tetracycline fibre plus scaling group and scaling alone group $(p=0.001)$.

Bleeding on probing: $91.4 \%$ of the sites bled on probing at the pre-treatment visit. Significant testing revealed that all treatment modalities were effective in reducing the number of periodontal sites which bled on probing when compared to the initial visit $(p<0.001)$

Plaque Index: Due to the strict oral hygiene, maintenance plaque index scores were low. The average plaque index did not significantly differ among the treatment groups at the baseline $(p=0.575)$, and after the treatment $(p=0.987)$. 
Table 3.10 Comparison of attachment level and pocket depth and gingival volume between different treatments using analysis of variance (ANCOVA), with subjects as unit of analysis. Paired t-test was used to compare before vs. after treatment.

\begin{tabular}{|c|c|c|c|c|}
\hline & $\mathrm{n}$ & Mean dif & SEM & p value \\
\hline \multicolumn{5}{|l|}{ Attachment level (mm) } \\
\hline Scaling \& TC fibre & 13 & 0.75 & 0.12 & $<0.001$ \\
\hline Scaling \& Min gel & 13 & 0.40 & 0.14 & 0.013 \\
\hline Scaling \& Met gel & 13 & 0.59 & 0.14 & 0.001 \\
\hline $\mathrm{S} \& \mathrm{R}$ alone & 13 & 0.26 & 0.11 & 0.032 \\
\hline ANCOVA $p$ value & & 0.048 & & \\
\hline \multicolumn{5}{|l|}{ Pocket depth (mm) } \\
\hline Scaling \& TC fibre & 13 & 1.35 & 0.17 & $<0.001$ \\
\hline Scaling \& Min gel & 13 & 0.81 & 0.13 & $<0.001$ \\
\hline Scaling \& Met gel & 13 & 0.97 & 0.18 & $<0.001$ \\
\hline$S \& R$ alone & 13 & 0.60 & 0.14 & $<0.001$ \\
\hline ANCOVA $p$ value & & 0.011 & & \\
\hline \multicolumn{5}{|l|}{ GCF Volume $(\mu 1)$} \\
\hline Scaling \& TC fibre & 13 & 0.10 & 0.08 & 0.21 \\
\hline Scaling \& Min gel & 13 & 0.02 & 0.04 & 0.70 \\
\hline Scaling \& Met gel & 13 & -0.05 & 0.05 & 0.33 \\
\hline$S \& R$ alone & 13 & 0.06 & 0.05 & 0.20 \\
\hline ANCOVA $p$ value & & 0.293 & & \\
\hline
\end{tabular}

* after correction the significance threshold was $<0.02$

Tc fibre= Tetracycline fibre

Min gel= Minocycline gel

Met gel= Metronidazole gel

$\mathrm{S} \& \mathrm{R}=$ Scaling and Root planing 
GCF volume: Although all the different treatments, except the metronidazole gel treatment, were effective in reducing the volume of GCF in the treated sites, but none of the changes were significant. In addition, the change in the GCF volume was not statistically different between the different treatments $(p=0.293)$ (Table 3.10).

Attachment level: Mean difference and SEM of attachment level in each group are shown in Table 3.10. Paired $t$ tests showed no significant improvement at the reassessment in all treatment groups. Analysis of covariance failed to show significant differences between different treatment groups on both site and subject based analysis ( $p=0.048)$.

$3.2 \cdot 2$

Change in the GCF stromelysin level after
adjunctive treatment with different
locally delivered antibiotics or scaling
and root planing alone

SL was detected in $40(19.4 \%)$ sites at reassessment. The enzyme level did not reach the detectable levels in any site in $28(53.8 \%)$ subjects at the reassessment visits.

The mean GCF level of SL decreased in all antibiotic treated groups and was statistically significantly decreased in the tetracycline fibre plus scaling group 
Table 3.11 GCF SL Mean reduction after therapy in four treatment groups, with site as the unit of analysis.

\begin{tabular}{|c|c|c|c|c|c|}
\hline & \multicolumn{3}{|c|}{ stromelysin (pg/30s) } & \multicolumn{2}{|c|}{ TIMP (pg/30s) } \\
\hline & $\mathbf{n}$ & mean dif & $\vec{p}$ & mean dif & $\bar{p}$ \\
\hline$S \&$ TC fibre & 51 & $0.11(0.05)$ & 0.020 & $-0.32(0.07)$ & 0.0001 \\
\hline S \& Min gel & 52 & $0.13(0.06)$ & 0.023 & $-0.22(0.08)$ & 0.0051 \\
\hline S \& Met gel & 52 & $0.01(0.30)$ & 0.730 & $-0.38(0.07)$ & $<0.001$ \\
\hline $\mathbf{S} \& \mathbf{R}$ alone & 51 & $-0.01(0.06)$ & 0.940 & $0.05(0.07)$ & 0.490 \\
\hline ANOVA $\mathrm{p}$ value & & 0.121 & & $<0.001$ & \\
\hline \multicolumn{6}{|c|}{$\begin{array}{l}\text { * Paired t-test, baseline and post-thera } \\
\text { \# Log transformed data } \\
\text { S \& Tc fibre= Scaling \& Tetracycline fib } \\
\text { S \& Met gel= Scaling \& Minocycline gel } \\
\text { S \& Min gel= Scaling \& Metronidazole gel } \\
\text { S \& R= Scaling and Root planing }\end{array}$} \\
\hline
\end{tabular}


Table 3.12 GCF SL mean reduction after therapy in four treatment groups, with subject as the unit of analysis.

\begin{tabular}{|c|c|c|c|c|c|}
\hline & \multicolumn{3}{|c|}{ stromelysin (pg/30s) } & \multicolumn{2}{|c|}{ TIMP (pg/30s) } \\
\hline & $\mathbf{n}$ & mean dif & p & mean dif & $\vec{p}$ \\
\hline$S \&$ TC fibre & 13 & $0.16(0.82)$ & 0.072 & $-0.33(0.08)$ & $<0.001$ \\
\hline s \& Min gel & 13 & $0.17(0.11)$ & 0.150 & $-0.24(0.10)$ & 0.03 \\
\hline S \& Met gel & 13 & $0.02(0.05)$ & 0.740 & $-0.38(0.08)$ & $<0.001$ \\
\hline$S$ \& $R$ alone & 13 & $-0.04 \quad(0.07)$ & 0.560 & $0.07(0.11)$ & 0.560 \\
\hline ANOVA $p$ value & & 0.174 & & 0.010 & \\
\hline $\begin{array}{l}\text { * Paired t-test, } \\
\# \text { Log transforme } \\
S \& \text { TC fibre= Sc } \\
S \& \text { Met gel= Sca } \\
S \& \text { Min gel= Sca } \\
S \& R=\text { Scaling a }\end{array}$ & $\begin{array}{l}\text { dat } \\
\text { ling } \\
\text { ing } \\
\text { ing } \\
\text { d Ro }\end{array}$ & $\begin{array}{l}\text { ine and post-th } \\
\text { \& Tetracycline } \\
\text { Minocycline ge } \\
\text { Metronidazole } \\
\text { t planing }\end{array}$ & py for & ach treatment & \\
\hline
\end{tabular}


$(p=0.020)$ and the minocycline gel plus scaling group $(p=0.023)$ (Table 3.11). If the patient was considered as the unit of analysis, it was revealed that GCF mean levels of SL did not decrease significantly after treatment in any group but was almost significant in the tetracycline fibre plus scaling treatment group $(p=0.072)$ (Table 3.12). The analysis of covariance (ANCOVA) on the baseline SL was used to reduce the error resulting from the variation in the baseline, i.e. to adjust out, mathematically, for the small differences at the baseline that might exist. When data was analysed both on the site and subject basis, the level of SL at baseline had significant effects on the response to treatment $(p<0.001)$, and this effect was positive i.e. the higher the baseline SL, the higher the reduction of SL. After the mean differences were adjusted for baseline values, the mean reduction in both the tetracycline fibre plus scaling and minocycline gel plus scaling groups were still considerably higher than those of the metronidazole gel plus scaling and scaling alone groups (Table 3.13). The treatment also had a significant effect on the reduction of SL $(p=0.001)$. Follow-up analysis showed significant differences between i) tetracycline fibre plus scaling and the scaling alone group $(p=0.004)$, ii) minocycline gel plus scaling and the scaling alone group $(p=0.002)$ (Table 3.13). However, if the patient was considered as the unit of analysis, there was no 
Table 3.13 Statistical analysis of changes of GCF level of Stromelysin between test groups $(n=206)$, with site as the unit of analysis.

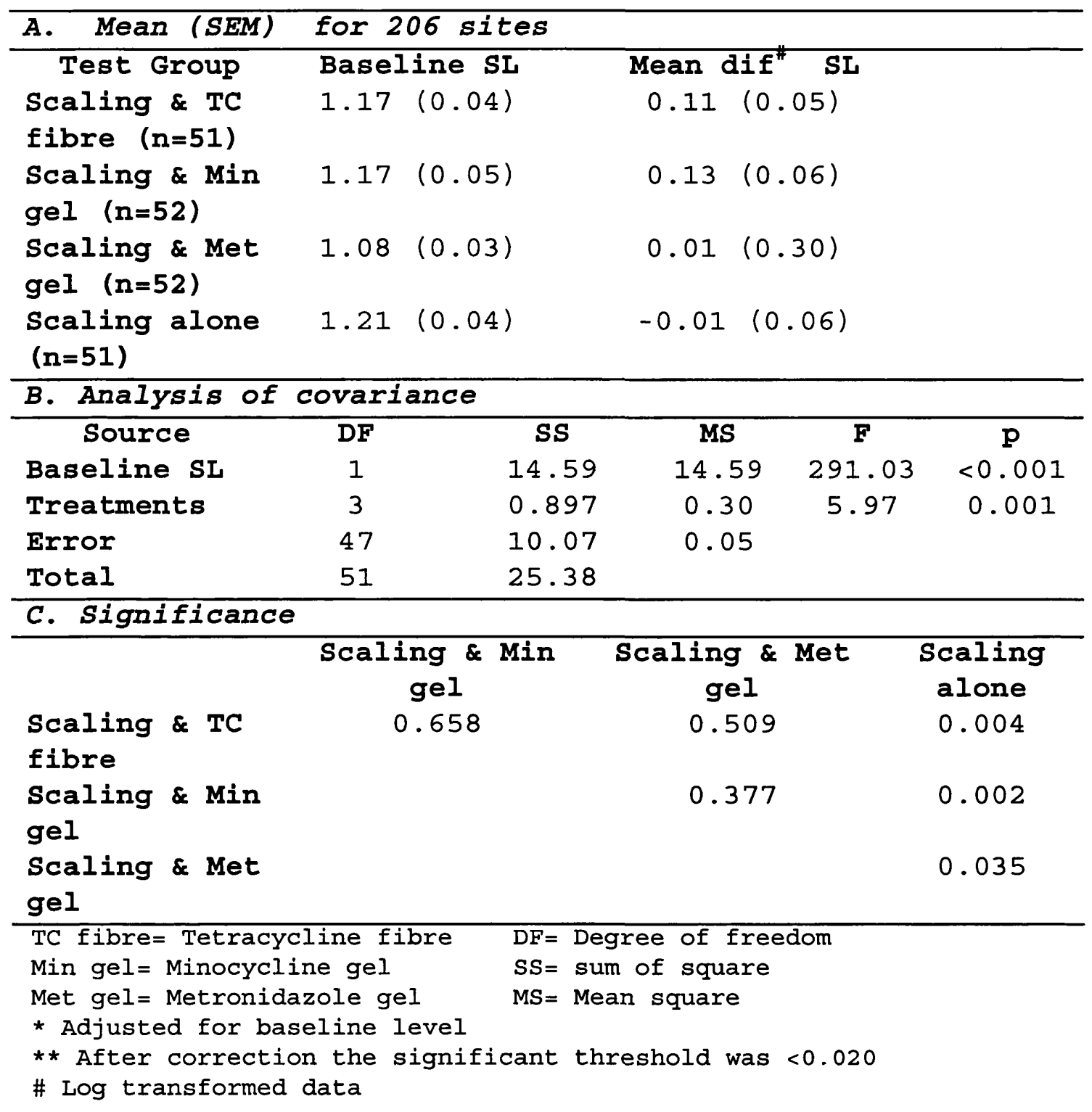


Table 3.14 Statistical analysis of changes of GCF level of stromelysin between test groups $(n=52)$, with subject as the unit of analysis.

\begin{tabular}{|c|c|c|c|c|c|}
\hline A. Mean (SEM) & \multicolumn{2}{|c|}{ for 52 patients } & & & \\
\hline Test Group & \multicolumn{2}{|c|}{$\begin{array}{l}\text { Baseline SL } \\
1.24(0.07)\end{array}$} & Mean dif ${ }^{\#}$ SL & & \\
\hline $\begin{array}{l}\text { Scaling \& } T C \\
\text { fibre }(n=13)\end{array}$ & 1.24 & $(0.07)$ & 0.1610 & 82) & \\
\hline $\begin{array}{l}\text { Scaling \& Min } \\
\text { gel }(n=13)\end{array}$ & 1.25 & $(0.09)$ & 0.1710 & 11) & \\
\hline $\begin{array}{l}\text { Scaling \& Met } \\
\text { gel }(n=13)\end{array}$ & 1.11 & $(0.04)$ & $0.07 \quad(0$ & 05) & \\
\hline $\begin{array}{l}\text { Scaling alone } \\
(n=13)\end{array}$ & 1.26 & $(0.07)$ & $-0.04 i$ & $.07)$ & \\
\hline B. Analysis of & covari & ance & & & \\
\hline Source & $\overline{D F}$ & SS & MS & $\mathbf{F}$ & $p$ \\
\hline Baseline SL & 1 & 2.04 & 2.04 & 48.26 & $<0.001$ \\
\hline Treatments & 3 & 0.44 & 0.15 & 3.47 & 0.023 \\
\hline Error & 47 & 1.99 & 0.04 & & \\
\hline Total & 51 & 4.46 & & & \\
\hline
\end{tabular}

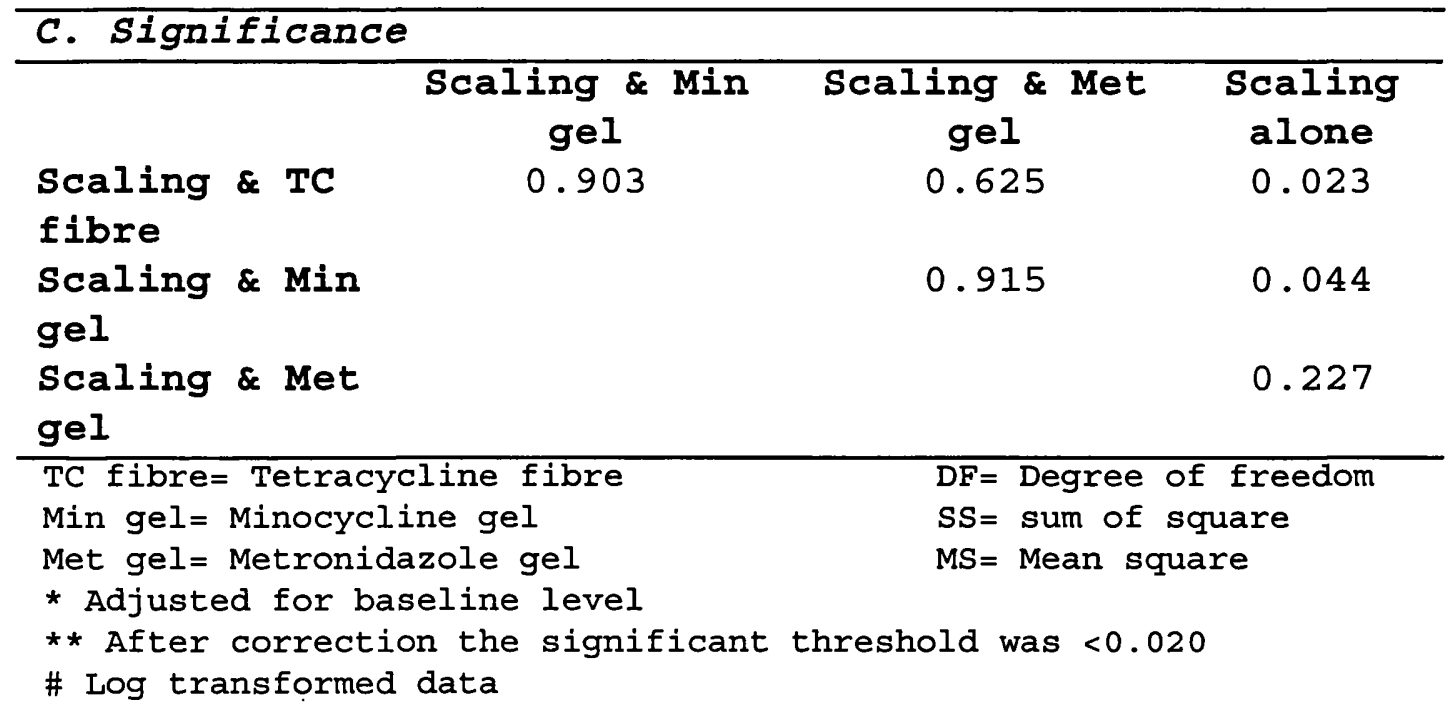


significant difference between treatment groups (Table $3.14)$.

3.2 .3

Change in the GCF tissue inhibitor
metalloproteinase level (TIMP) after
adjunctive treatment with different locally
delivered antibiotics or scaling and root
planing alone

TIMP was detected in $205(99.5 \%)$ sites. Paired sample ttest demonstrated that the level of GCF TIMP significantly increased in all antibiotic treatment groups, whereas it decreased in scaling groups although not significantly $(\mathrm{p}=0.49)$. One-way analysis of variance demonstrated a significant difference among the groups for changes in the GCF TIMP levels $(p<0.001)$ (Table 3.11). When Tukey's test was employed to determine direction and location of difference, it showed that all antibiotic therapy groups were significantly different from the scaling alone group. However, no significant difference was found between antibiotic therapy groups (Table 3.11 ). The results of the subject basis analysis was similar to those of site basis analysis (Table 3.12). When data was analysed on the site basis, ANCOVA showed that the level of TIMP at the baseline had a significant effect on the response to treatment $(p=0.007)$, i.e. regardless of treatment type, the higher 
Table 3.15 Statistical analysis of changes of GCF level of TIMP between test groups $(n=206)$, with site as the unit of analysis.

\begin{tabular}{|c|c|c|c|}
\hline \multicolumn{4}{|c|}{ A. Mean (SEM) for 206 sites } \\
\hline Test Group & Baseline TIMP & \multicolumn{2}{|l|}{ Mean dif ${ }^{\#}$ TIMP } \\
\hline $\begin{array}{l}\text { Scaling \& } T C \\
\text { fibre }(n=51)\end{array}$ & $2.12(0.06)$ & -0.32 & \\
\hline $\begin{array}{l}\text { Scaling \& Min } \\
\text { gel }(\mathrm{n}=52)\end{array}$ & $2.30 \quad(0.06)$ & -0.22 & \\
\hline $\begin{array}{l}\text { Scaling \& Met } \\
\text { gel }(n=52)\end{array}$ & $2.19(0.06)$ & -0.38 & \\
\hline $\begin{array}{l}\text { Scaling alone } \\
(n=51)\end{array}$ & $2.39(0.06)$ & 0.05 & \\
\hline \multicolumn{4}{|c|}{ B. Analysis of covariance } \\
\hline Source & $\overline{\mathrm{DF}}$ & MS & $\mathbf{p}$ \\
\hline $\begin{array}{l}\text { Baseline } \\
\text { TIMP }\end{array}$ & \multicolumn{3}{|c|}{ TIMP } \\
\hline Treatments & 2.13 & 0.71 & 0.007 \\
\hline Error & 34.61 & \multirow[t]{2}{*}{0.17} & \\
\hline Total & 60.73 & & \\
\hline \multicolumn{4}{|l|}{ C. Significance } \\
\hline & $\begin{array}{c}\text { Scaling \& Min } \\
\text { gel }\end{array}$ & $\begin{array}{c}\text { Scaling \& Met } \\
\text { gel }\end{array}$ & $\begin{array}{l}\text { Scaling } \\
\text { alone }\end{array}$ \\
\hline $\begin{array}{l}\text { Scaling \& } T C \\
\text { fibre }\end{array}$ & 0.503 & 0.170 & 0.036 \\
\hline \multirow{2}{*}{\multicolumn{2}{|c|}{$\begin{array}{l}\text { gel } \\
\text { Scaling \& Met } \\
\text { gel }\end{array}$}} & 0.400 & 0.018 \\
\hline & & & 0.001 \\
\hline \multicolumn{2}{|c|}{$\begin{array}{l}\text { TC fibre= Tetracycline fibre } \\
\text { Min gel= Minocycline gel } \\
\text { Met gel= Metronidazole gel } \\
\text { * Adjusted for baseline level }\end{array}$} & $\begin{array}{r}\text { DF }=\text { Degree of } \\
\mathrm{SS}=\text { Sum of } \mathrm{squ} \\
\mathrm{MS}=\text { Mean squar } \\
\text { hold was }<0.020\end{array}$ & $\begin{array}{l}\mathrm{DF}=\text { Degree of freedom } \\
\mathrm{SS}=\text { Sum of square } \\
\mathrm{MS}=\text { Mean square }\end{array}$ \\
\hline
\end{tabular}


Table 3.16 Statistical analysis of changes of GCF level of TIMP between test groups $(n=52)$, with subjects as the unit of analysis.

\begin{tabular}{|c|c|c|c|c|}
\hline A. Mean (SEM) & for 52 & patients & & \\
\hline Test Group & Baseli & ine TIMP & Mean dif & \\
\hline $\begin{array}{l}\text { Scaling \& TC } \\
\text { fibre }(n=13)\end{array}$ & 2.20 & $(0.09)$ & $-0.33 \quad(0.08$ & \\
\hline $\begin{array}{l}\text { Scaling \& Min } \\
\text { gel }(n=13)\end{array}$ & 2.38 & $(0.09)$ & $-0.24 \quad(0.10)$ & \\
\hline $\begin{array}{l}\text { Scaling \& Met } \\
\text { gel }(n=13)\end{array}$ & 2.30 & $(0.09)$ & $-0.38 \quad(0.08)$ & \\
\hline $\begin{array}{l}\text { Scaling alone } \\
(n=13)\end{array}$ & 2.53 & $(0.08)$ & $0.07(0.11)$ & \\
\hline \multicolumn{5}{|c|}{ B. Analysis of covariance } \\
\hline Source & DF & SS & MS & $\mathbf{p}$ \\
\hline \multicolumn{5}{|l|}{ TIMP } \\
\hline Treatments & 3 & 0.43 & 2.23 & 0.097 \\
\hline Error & 47 & 3.05 & 0.06 & \\
\hline Total & 51 & 6.65 & & \\
\hline $\begin{array}{l}\text { TC fibre }=\text { Tetracy } \\
\text { Min gel = Minocycl } \\
\text { Met gel= Metronid } \\
\text { * Adjusted for ba } \\
\text { * After correcti } \\
\# \text { Log transformed }\end{array}$ & $\begin{array}{l}\text { line fibr } \\
\text { ne gel } \\
\text { zole gel } \\
\text { eline lev } \\
\text { n the sig } \\
\text { data }\end{array}$ & nificant & $\begin{array}{l}\text { DF }=\text { Degree of } \\
\mathrm{SS}=\text { Sum of } \mathrm{squ} \\
\mathrm{MS}=\text { Mean squar } \\
\text { old was }<0.020\end{array}$ & $\begin{array}{l}\text { reedom } \\
\text { ire }\end{array}$ \\
\hline
\end{tabular}


the TIMP level at the baseline, the higher the change of TIMP level after treatment. Results revealed a significant difference between the minocycline gel plus scaling and the scaling alone group $(p=0.018)$, and also between metronidazole gel plus scaling and scaling alone group $(p=0.001) \quad($ Table 3.15). However, if the patient was considered as the unit of analysis, there was no significant difference between the different treatments $(p=0.097) \quad($ Table 3.16$)$.

\section{2 .4}

The effects of smoking, sex, site, and age on changes of GCF SL, TIMP, and volume

The effects of smoking status, location of site, sex, and age on changes of GCF SL are presented in Table 3.17. None of these factors had any significant effect on SL level. Nevertheless, the change in SL level was lower in smokers, females, subjects less than 35 years and posterior sites, compared to non-smokers $(p=0.20)$, males $(p=0.12)$, subjects over 35 years $(p=0.79)$ and anterior sites $(p=0.65)$ respectively.

The effects of smoking, sex, site, and age on the changes of GCF TIMP were demonstrated to be insignificant. The change in GCF TIMP was lower in non-smokers, females, subjects over 35 years and anterior sites compared to 
Table 3.17 The effects of smoking, sex, site and age on changes of GCF SL, TIMP and volume after treatment. Sites was used as the unit of analysis $(n=206)$. Two sample $t$ test was utilised. Means \& (SEM) are given.

\begin{tabular}{|c|c|c|c|c|c|c|c|c|}
\hline & \multicolumn{3}{|c|}{ smoking } & \multicolumn{2}{|l|}{$\operatorname{sex}$} & $\overline{g e}$ & \multicolumn{2}{|c|}{ site } \\
\hline$\Delta$ SI $^{*}$ & $\begin{array}{l}\text { Sm } \\
\mathrm{n}=115 \\
0.03 \\
(0.03)\end{array}$ & $\begin{array}{l}\text { non sm } \\
n=91 \\
0.1 \\
(0.04)\end{array}$ & $\begin{array}{c}\quad F \\
n=128 \\
0.03 \\
(0.05)\end{array}$ & $\begin{array}{c}\mathbf{M} \\
\mathbf{n}=78 \\
0.11 \\
(0.04)\end{array}$ & $\begin{array}{c}<35 \\
n=28 \\
0.05 \\
(0.04)\end{array}$ & $\begin{array}{c}>35 \\
n=178 \\
0.06 \\
(0.03)\end{array}$ & $\begin{array}{l}\text { Ant } \\
n=145 \\
0.08 \\
(0.04)\end{array}$ & $\begin{array}{l}\text { Post } \\
n=61 \\
0.06 \\
(0.03)\end{array}$ \\
\hline$p$ & \multicolumn{2}{|c|}{0.20} & \multicolumn{2}{|c|}{0.12} & \multicolumn{2}{|c|}{0.79} & \multicolumn{2}{|c|}{0.65} \\
\hline$\Delta$ TIMP & $\begin{array}{r}-0.28 \\
(0.05)\end{array}$ & $\begin{array}{l}-0.14 \\
(0.06)\end{array}$ & $\begin{array}{l}-0.21 \\
(0.05)\end{array}$ & $\begin{array}{r}-0.24 \\
(0.06)\end{array}$ & $\begin{array}{l}-0.27 \\
(0.01)\end{array}$ & $\begin{array}{l}-0.21 \\
(0.04)\end{array}$ & $\begin{array}{l}-0.21 \\
(0.04)\end{array}$ & $\begin{array}{l}-0.22 \\
(0.07)\end{array}$ \\
\hline$p$ & \multicolumn{2}{|c|}{0.08} & \multicolumn{2}{|c|}{0.69} & \multicolumn{2}{|c|}{0.59} & \multicolumn{2}{|c|}{0.97} \\
\hline $\begin{array}{l}\text { पGCF } \\
\text { vol }\end{array}$ & $\begin{array}{l}0.01 \\
(0.03)\end{array}$ & $\begin{array}{c}0.04 \\
(0.04)\end{array}$ & $\begin{array}{l}0.01 \\
(0.03)\end{array}$ & $\begin{array}{l}0.06 \\
(0.04)\end{array}$ & $\begin{array}{l}-0.02 \\
(0.08)\end{array}$ & $\begin{array}{l}0.03 \\
(0.03)\end{array}$ & $\begin{array}{l}0.02 \\
(0.03)\end{array}$ & $\begin{array}{l}0.03 \\
(0.05)\end{array}$ \\
\hline$p$ & 0.5 & & & 340 & & 490 & & 910 \\
\hline
\end{tabular}

\# Log transformed data

Table 3.18 Correlation of PD and GCF Vol with SL and TIMP at the reassessment visit, with the site as the unit of analysis $(n=206)$.

\begin{tabular}{|c|c|c|}
\hline & Stromelysin (pg/30s) & TIMP $(\mathrm{pg} / 30 / \mathrm{s})$ \\
\hline $\operatorname{Vol}(\mu \mathrm{L})$ & $r=0.108 \quad(0.049)$ & $0.389 \quad(\mathrm{p}<0.001)$ \\
\hline $\mathrm{PD} \quad(\mathrm{mm})$ & $r=0.188 \quad(p=0.058)$ & $0.194 \quad(p=0.068)$ \\
\hline
\end{tabular}


smokers subjects $(p=0.08)$; males $(p=0.69)$; subjects less 35 years $(p=0.59)$ and posterior sites $(p=0.97)$ respectively (Table 3.17). These changes were almost completely opposite to those reported for SL alone.

The changes of mean GCF volume was lower in smokers, females and anterior sites compared to non-smokers $(p=0.56) ;$ male $(p=0.34)$ and posterior sites $(p=0.91)$ respectively. The mean GCF volume decreased slightly in subjects over 35 years but it increased slightly in subjects less than $35(p=0.49)$ (Table 3.17).

General linear model procedures (GLM) was used to take into account the effect of factors, such as smoking, sex, and site, while examining the effect of treatment on the changes of SL or TIMP. The results showed none of the individual effects were significant ( $p$ value for smoking $=0.101, \operatorname{sex}=0.136$, sites $=0.686$, age $=0.416$ ).

$3 \cdot 2 \cdot 5$

Correlation between pocket depth, GCF volume and GCF levels of SL and its inhibitor at reassessment

The mean levels of SL and TIMP showed positive associations with the mean GCF volume and pocket depth. However, the correlations were low (Table 3.18). 


\section{Prediction of response to treatment according to the baseline level of stromelysin and tissue inhibitor of metalloproteinases}

In order to determine the success of treatment, all sites were examined for bleeding on probing and pocket depth at the reassessment visit. Treatments were considered successful, when the sites did not show bleeding on probing and had a pocket depth of equal or less than $3.5 \mathrm{~mm}$. Overall twenty six $(12.6 \%)$ sites were found which showed both criteria for treatment success at the reassessment examination, whereas one hundred and seventeen (56.8\%) sites were detected which still had bleeding on probing and pocket depth over $3.5 \mathrm{~mm}$. The former category was assigned as the 'best responding sites' (BRS) and the latter as the 'worst responding sites' (WRS). The baseline levels of stromelysin, and TIMP in the best responding sites (BRS) $(n=26)$ and the worst responding sites (WRS) ( $n=117)$ were compared in order to investigate their ability to predict treatment outcome. The Mann-Whitney $U$ test was made to compare the baseline GCF SL between the WRS and BRS, and the two sample t-test was used to compare the baseline GCF TIMP between the WRS and BRS. The changes in GCF TIMP and SL between the WRS and the BRS was subjected to the two sample t-test. 


\begin{abstract}
Prediction of response to treatment according to the baseline levels of stromelysin and comparison of BRS and WRS in their change of stromelysin levels.
\end{abstract}

Table 3.19 presents the level of GCF SL, and TIMP as well as volume at the BRS and WRS at the baseline. The mean GCF levels of SL at the baseline failed to differentiate significantly between sites without $B O P$ and $P D \leq 3.5 \mathrm{~mm}$ (BRS) and those with BOP and pocket depth of more than $3.5 \mathrm{~mm}$ (WRS) (Mann-Whitney $U$ test, $\mathrm{p}=0.459)$. However, the results showed that the mean level of SL in the BRS was lower than the WRS at the baseline. There was also no significant difference between the WRS and the BRS in the level of SL change $(p=0.16)$. However, SL decreased three times more in the BRS than the WRS but due to the presence of large variability no significant difference was demonstrated. The comparison of the WRS and the BRS, broken down by their treatments, indicated that in all treatment groups the post-treatment reduction of SL was higher in the BRS than the WRS, although it was not statistically significant (Table 3.20). 
Table 3.19 Comparison of GCF stromelysin (SL), GCF TIMP, volume (Vol) at the baseline examination between BRS and WRS, using Mann-Whitney $U$ test for GCF SL, Two sample t-test for GCF TIMP and volume. Mean \& (SEM) are given.

\begin{tabular}{l|ccccc}
\hline & BRS $(n=26)$ & WRS $(n=117)$ & P value \\
\hline SL & 18.82 & $(2.66)$ & 20.44 & $(2.52)$ & 0.459 \\
TIMP & 263.2 & $(51.3)$ & 317.9 & $(35.1)$ & 0.410 \\
Vol & 0.441 & $(0.056)$ & 0.496 & $(0.033)$ & 0.706 \\
\hline
\end{tabular}

Table 3.20 Comparison of changes of GCF stromelysin (SL), GCF TIMP, volume (Vol) between BRS and WRS, using Two sample t-test, Probability value and $\log _{10}$ transformed mean \& (SEM) are given.

\begin{tabular}{l|ccccc}
\hline & BRS $(n=26)$ & WRS $(n=117)$ & p value \\
\hline$\Delta S L$ & 0.14 & $(0.06)$ & 0.04 & $(0.03)$ & 0.16 \\
$\Delta T I M P$ & -0.22 & $(0.16)$ & -0.21 & $(0.05)$ & 0.99 \\
$\Delta V O I$ & 0.13 & $(0.07)$ & -0.01 & $(0.35)$ & 0.09 \\
\hline
\end{tabular}


Prediction of response to treatment according to the baseline level of TIMP and comparison of BRS and WRS in their change of TIMP levels.

The two sample t-test did not show any significant difference between the mean baseline TIMP level in the BRS and the WRS (two sample t-test, $\mathrm{p}=0.410$ ); Although the GCF level of TIMP was demonstrated to be lower in the BRS than the WRS at baseline (Table 3.19). Statistical analysis of changes in GCF TIMP failed to show any significant difference between BRS and WRS overall ( $p=0.99)$ (Table 3.20). Comparison between changes of TIMP in different groups of treatment revealed that in all the treatment groups, the TIMP changes were higher in BRS compared to WRS (Table 3.21), although that change was not significant.

Prediction of response to treatment (changes in the attachment levels) according to the baseline GCF levels of stromelysin and TIMP

All the sites were broken down according to their change in attachment levels; a) sites with attachment gain $>1 \mathrm{~mm}, \mathrm{~b}$ ) sites with attachment gain between zero and $1 \mathrm{~mm}$, and c) sites with attachment loss. The change in the SL and TIMP level were compared in the sites with attachment gain $>1 \mathrm{~mm}$ 
Table 3.21 Comparison of changes of GCF stromelysin (SL) and GCF TIMP between BRS and WRS in four different treatments, using Two sample t-test. Log transformed mean difference \& (SEM) are given.

\begin{tabular}{|c|c|c|c|c|c|}
\hline & $n \cdot B R S$ & $\overline{B R S}$ & n.WRS & WRS & p value \\
\hline $\begin{array}{l}\text { Tetracycline } \\
\text { Fibre }\end{array}$ & 9 & & 21 & & \\
\hline$\Delta$ stromelysin & & $\begin{array}{c}0.14 \\
(0.07)\end{array}$ & & $\begin{array}{c}0.08 \\
(0.08)\end{array}$ & 0.08 \\
\hline$\triangle T I M P$ & & $\begin{array}{l}-0.38 \\
(0.29)\end{array}$ & & $\begin{array}{c}0.32 \\
(0.11)\end{array}$ & 0.86 \\
\hline Minocycline Gel & 5 & & 30 & & \\
\hline$\Delta$ Stromelysin & & $\begin{array}{c}0.29 \\
(0.13)\end{array}$ & & $\begin{array}{c}0.11 \\
(0.06)\end{array}$ & 0.24 \\
\hline$\triangle T I M P$ & & $\begin{array}{l}-0.27 \\
(0.32)\end{array}$ & & $\begin{array}{l}-0.26 \\
(0.08)\end{array}$ & 0.98 \\
\hline $\begin{array}{l}\text { Metronidazole } \\
\text { Gel }\end{array}$ & 6 & & 31 & & \\
\hline$\Delta$ stromelysin & & $\begin{array}{c}0.06 \\
(0.06)\end{array}$ & & $\begin{array}{c}0.01 \\
(0.04)\end{array}$ & 0.44 \\
\hline$\triangle T I M P$ & & $\begin{array}{l}-0.43 \\
(0.36)\end{array}$ & & $\begin{array}{l}-0.38 \\
(0.08)\end{array}$ & 0.90 \\
\hline $\begin{array}{l}\text { Scaling \& Root } \\
\text { planing }\end{array}$ & 6 & & 35 & & \\
\hline$\Delta$ stromelysin & & $\begin{array}{c}0.08 \\
(0.19)\end{array}$ & & $\begin{array}{c}0.01 \\
(0.08)\end{array}$ & 0.74 \\
\hline$\Delta T I M P$ & & $\begin{array}{c}0.28 \\
(0.15)\end{array}$ & & $\begin{array}{c}0.04 \\
(0.09)\end{array}$ & 0.21 \\
\hline
\end{tabular}


and the sites with attachment loss. 43 sites (20.87\%) had attachment gain from the baseline level, whereas 53 sites $(25.73 \%)$ showed attachment loss from the baseline.

Mann-Whitney $U$ test failed to distinguish between the mean GCF SL of the attachment gaining sites (AGS) and the attachment losing sites (ALS) at the baseline (Mann-Whitney $\mathrm{U}$ test, $\mathrm{p}=0.84$ ) (Table 3.22). There was no significant difference between AGS and ALS sites at the reassessment visit, when they were compared for their mean baseline levels of TIMP (two sample t-test, $p=0.63$ ) (Table 3.23). Therefore, the TIMP and SL mean baseline were not able to predict the response to the treatment as defined by attachment level changes. 
Table 3.22 Comparison of GCF stromelysin (SL), GCF TIMP, volume (Vol) at the baseline examination between sites with attachment gain $(\triangle A L>1)$ and sites with attachment loss $(\triangle \mathrm{A} l<0)$, using Mann-Whitney $U$ test for GCF SL, two sample t-test for GCF TIMP, and volume.

\begin{tabular}{l|ccccc}
\hline & \multicolumn{2}{|c}{ AGS $(\mathbf{n = 4 3 )}$} & ALS $(n=53)$ & p value \\
\hline SL & 17.82 & $(2.05)$ & 28.06 & $(7.87)$ & 0.84 \\
TIMP & 247.3 & $(55.9)$ & 235.5 & $(37.5)$ & 0.63 \\
Vol & 0.442 & $(0.05)$ & 0.445 & $(0.05)$ & 0.91 \\
\multicolumn{2}{l}{ AGS= Attachment gain sites } \\
ALS= Attachment loss sites
\end{tabular}


CHAPTER 4

DISCUSSION 
The study was designed as a parallel study to avoid cross over effects between different antibiotic treatments. In order to avoid bias in judgement and provide a basis for the standard methods of statistical analysis, patients were randomly allocated to four different groups, thus each patient had a 25\% chance of being assigned to treatment. The subjects in four treatment groups were balanced on the basis of factors suspected to have an influence on the outcome such as smoking and age.

The clinical design of this experiment allowed evaluation of the effect of three different locally delivered antibiotics on the level of SL and TIMP GCF.

\section{2}

The choice of biochemical parameters

The reason for selecting fibroblast SL in this study was that SL has been shown to be a marker of stromal cell involvement in the process of tissue degradation (BirkedalHansen et al., 1993) and it has also been suggested that SL may play a role in chronic inflammatory periodontitis (Ingman et al., 1994a,b). The fibroblast type of SL is chosen because fibroblast-type collagenases have been shown to be constutively synthetised and secreted by fibroblasts, 
whereas the human neutrophil collagenase is stored in intra cellular specific granules, having been synthesised during neutrophil maturation in bone marrow. Thus, the levels of this MMP released by mature circulating neutrophils are regulated by factors that affect degranulation rather than biosynthesis of collagenase (Birkedal-Hansen, 1988). For this reason fibroblast stromelysin (MMP-3) was used in the study. Meanwhile, since it has been shown that systemic tetracycline administration does not inhibit well fibroblast derived MMPs (Golub et al., 1991), it was decided to utilise local delivery systems which provide high concentration of tetracycline that can inhibit all MMPs such as PMN and fibroblast origin.

4.3 Methodological considerations

4.3 .1

Quantification of gingival crevicular fluid stromelysin and tissue inhibitor of metalloproteinases

The enzyme linked immunosorbent assay (ELISA) used in this study was validated and proven suitable for the quantification of MMPs and TIMP in GCF and has several advantages over other methods. First, the ELISA assay system does not require radioactive materials which are expensive and hazardous. Second, the test is convenient, 
simple and can be carried out within a few hours. Third, it is suitable for dealing rapidly with a large number of samples.

The ELISA methodology has important technical aspects so that careful attention to experimental protocol is essential to ensure accurate and reproducible results. However, this method has some inherent problems such as, high background, edge effects and the hook effect, which may introduce erroneous results or make their interpretation difficult. Increasing the number of wash steps and also using a non specific protein i.e. bovine serum albumin (BSA) to bind unreacted sites in solid phase can prevent the high background in the ELISA technique. In these experiments the optical density (OD) for control wells were much lower than those obtained for both samples and standards, therefore this was not such a problem here. The use of inside wells for running samples and controls can prevent the edge effect of the ELISA technique as it has been reported that the outside wells of a plate may absorb more protein than the inside wells, resulting in assay bias (Tijssen, 1985; Carpenter, 1992). Low affinity antibody, inadequate washing and suboptimal concentrations of labelled antibody have been suggested as possible causes of the hook effect. This is an unexpected fall in the amount of substance at the end of the dose response curve, resulting in a gross underestimation of the substance 
(Carpenter, 1992). However, in the present experiments such an effect was not demonstrated.

In this study GCF data were reported as absolute amounts of GCF constituent per 30s sample, and does not include the volume of GCF in its calculation. This method may be subject to less variance and is probably less sensitive to alterations caused by evaporation or contamination of the sample which would appear to be unpredictable, irrespective of the sampling technique used. The rational for expressing results of GCF constituents as absolute amount (pg/30s) has been discussed extensively (Lamster et al., 1986, Lamster et al., $1988 \mathrm{a}, \mathrm{b})$.

In the present study paper strips placed just into the crevice for 30 seconds were utilised for GCF collection to minimise irritation and microleakage of the subepithelial microvasculature. Such a non-invasive method can provide a sample which is truly from a pool of biochemical compounds in the GCF, and has not been influenced by the influx of the enzymes and biochemical markers from the serum as a result of trauma to blood vessels during the sampling technique. 
Using the ANOVA it is possible to draw conclusions about only one parameter which is a variable of interest to the researcher. In this situation, to obtain a valid inference, all other parameters should ideally be similar across the test groups. Otherwise the differences in some parameters could have important influences on the outcome variable. In the biological studies it is practically impossible to obtain samples of subjects which differ from each other only in one parameter. Randomisation is a suitable compromise by evenly distributing the factors with likely influence among the test groups. Nevertheless serious imbalance in such factors is not unlikely and can damage severely the validity of the statistical analysis. Analysis of covariance was used in the present study with the change in biochemical parameters as the main effect and the baseline values as continues covariated. Inspection of data indicated a large variability among the baseline values. When uncorrected changes in SL and TIMP were compared between treatment groups using ANOVA no statistically significant difference was found. However, covariance values of changes in SL and TIMP adjusted were proved to be significant among treatment groups when ANCOVA was used for the site base data. This indicates that ANCOVA is a more powerful statistical method than ANOVA. 
Nowadays, there is evidence that host factors are crucial in periodontal diseases. The conclusion of longitudinal studies is that; a) the progression of destructive periodontitis is subject-related; b) in the absence of treatment, inflammatory periodontal lesions will not necessarily progress; and c) comparatively few individuals in a population show advanced periodontal breakdown (Goodson et al., 1982; Albandar et al., 1986). Therefore, the current view is that periodontal disease is subject related. However, it should be born in mind that host factors influence all sites within the same mouth but site responsiveness to the treatments differ from site to site which may be due to the different composition of microbiota, or the morphology of the lesion. Furthermore, variation exists between the activity of diseased sites, thus if a site is considered as the unit of observation and the subject is considered as the unit of analysis, the question arises as to the effect upon the latter of the vast majority of inactive sites.

This is important in analysis of biochemical parameters which have shown high variations between the sites. In addition, in our study the sites which showed attachment loss occurred in some sites in the different individuals. With regard to these considerations, data in this study were analysed with site and subject as the unit of analysis 
in order to consider both site specificity and subject specificity.

4.6

GCF metalloproteinases after locally

delivered antibiotic therapy

\subsubsection{A cross sectional analysis of the association between GCF SL and TIMP levels and clinical parameter at the baseline}

The large fluctuation in amount of GCF SL and TIMP within patient and sites may be due to variability in the pathogenicity of the microbiota at different sites. This may result in variation in local host response and thus, variation in the levels of biochemical mediators harvested from GCF.

Yanagimura et al. (1989) demonstrated that the collagenase activities in GCF from subjects receiving maintenance care was significantly lower than that observed in the nontherapy subjects. Haerian et al. (1994) found that the level of SL and TIMP were lower in maintenance patients. With regard to these studies, one of the reasons why GCF SL was detected in only $29.6 \%$ of sites, was that study patients in this investigation had already received periodontal treatment. Bunning et al. (1984) showed that 
the physiologic inhibitors $\alpha 2$-microglobulin and tissue inhibitor of metalloproteinases (TIMPs) inhibit the fibroblast collagenase more efficiently than neutrophil collagenase. It is highly likely that active sL reaching the crevice would readily bind to inhibitors and causes SL in GCF not to be detected by this technique.

TIMP was detected in $99 \%$ of sites, which could be related to the presence of other MMPs in the inflamed gingiva which together increase tissue destruction and stimulates TIMP production (Nomura, Takahashi \& Hara, 1993).

As bleeding on probing and suppuration are regarded as signs of periodontal disease (Haffajee, Socransky \& Goodson, 1983a; Badersten, Neilèus \& Egelberg, 1990), the level of SL and TIMP were compared in different groups of sites. Our results showed that the levels of SL in the GCF have a relation with gingival inflammation scores, and also the levels of SL were higher in sites with bleeding on probing than in sites with did not bleed on probing. However, in suppurative sites the level of SL was lower than non suppurative sites. These results provide some insight into the level of $\mathrm{SL}$ in $\mathrm{GCF}$ and is probably simply a measure of the degree of inflammation. It can possibly also be considered as a measure of net collagen breakdown or tissue destruction. 
Since the GCF SL showed a significant difference between gingival inflammation scores, and also a high level of SL was present in sites with bleeding on probing than sites with no bleeding on probing, SL may also be considered as a marker of inflammation.

The level of GCF TIMP increased after treatment; it can be speculated that the level of TIMP in GCF can be considered as a measure of health. This is consistent with those reported by Haerian et al. (1995b).

4.6 .2

Comparison between GCF level of SL, TIMP and volume considering smoking, gender, age, and anterior vs. posterior sites

Recent data from several sources indicate that demographic variables within a study population can influence factors associated with periodontal disease and disease characteristics. Previous studies have also shown that demographic factors also influence the periodontal microflora, which might be expected in as much as immune and inflammatory responses commonly are considered to be crucial in the containment of periodontal bacterial flora. 
The objective of the assessment of data in terms of smoking, gender, age, and position of sites was to detect whether these variables had any effect on GCF SL and TIMP.

Previous studies have shown that the association between smoking and increased prevalence and incidence rates of periodontal disease have suggested that smoking has a strong association with severity of periodontal disease. This could be explained by a number of biologic phenomena. Nicotine and its by-products have a vasoconstrictive effect, on peripheral gingival blood vessels (Baab, \& Oberg, 1987). In addition, smoking may reduce the functional activity of leukocytes and macrophages in saliva and crevicular fluid, and decrease chemotaxis and phagocytosis of blood and tissue polymorphonuclear (PMN) leukocytes, thereby depressing phagocyte-mediated protective responses to periodontal pathogens (Palmer, 1988). Grossi et al. (1994) reported less healing after mechanical treatment in smokers compared to non-smokers and showed this decreased clinical response was associated with less reduction of subgingival microorganisms. Data from changes of SL are consistent with the findings of Grossi et al. (1994) who showed higher clinical changes in nonsmokers. Our results also confirmed those of Baab \& Oberg (1987) who showed the GCF volume is lower in smokers compared to non-smokers. 
Our results demonstrated that the mean level of SL was lower in smokers than non-smokers whereas the mean levels of TIMP was higher in smokers compared to non-smokers. The mechanisms that lead to differences in the SL between smoker and non-smokers are unclear, but some hypotheses indicate that it may be due to dysfunction of polymorphonuclear (PMN) leukocytes which indirectly influence production of SL from fibroblasts. With regard to the results of Ding et al. (1994) who demonstrated low activities of MMPs GCF in periodontitis smokers compared to healthy smokers, the level of free TIMP in smokers is expected to be higher compared to non-smokers.

Our observations showed the level of SL was higher in subjects more than 35 years of age compared to subjects less than 35. However, the level of TIMP was higher in subjects less than 35 years compared to subjects more than 35 years. The changes were higher in subjects over 35 years whereas changes of TIMP were higher in subjects less than 35 years. Epidemiological evidence suggests that evidence of periodontal breakdown is increased with age. Grossi et al. (1994) demonstrated the prevalence of target periodontal pathogens based on species were different in different ages. This study also showed subjects in younger age categories were at lower risk for clinical attachment loss and also the risk of attachment loss were greater in males compared to females. Aass, Tollefsen \& Gjermo (1994) 
showed that the frequency of subjects with radiographic alveolar bone loss increased significantly with age. Ismail et al. (1990) showed that age is a risk factor or predictor of loss of periodontal attachment. Papapanou et al. (1990) suggested that loss of periodontal attachment is more related to baseline loss of periodontal attachment than to age. There is no evidence in these studies to show whether these changes are due to increased experience of periodontal disease or are part of the ageing process or both.

Grossi et al. (1994) demonstrated that the prevalence of the target periodontal pathogens were greater in males than females. Our results showed that the level of SL and TIMP were higher in males than females and also changes of these enzymes were higher in males than females. There is no evidence to show any sex differences between MMPs GCF. In addition the higher level of GCF volume in males than females can be due to higher plaque index scores and gingival inflammation scores in males compared to females.

Previous studies have shown that molar teeth with furcation involvement, responded less well to treatment compared to premolars and anterior teeth (Nordland et al., 1987, Loos et al., 1989). However, it could be due to difficulty in removal of subgingival plaque and calculus in molar furcation areas and poor accessibility of instruments to 
furcation areas. Our results showed that changes of SL were higher in anterior sites, however it cannot be established that site position alone is a marker of response to treatment.

The efforts were made to disclose the relationship of these enzymes to demographic parameters which are supposed to have an effect on the prevalence and response to treatment of periodontal disease.

Since the previous studies showed the level of SL activity and also the amount of this enzyme may serve as an indicator of patients with periodontal disease, discriminate analysis was performed to discriminate the sites at which SL were detected, and also sites with high and low levels of TIMP, by clinical and demographic parameters. Among 11 variables only the GCF volume and TIMP levels were able to differentiate between sites with and sites without detectable SL. The GCF volume and SL level and sex could discriminate sites with high level of TIMP from sites with low level of TIMP. This indicates that, it is difficult for the clinical parameters to discriminate the different levels of biochemical parameters in GCF. 
Antibiotics are often advocated as useful adjuncts in periodontal therapy based on their effectiveness against periodontopathogens (Lindhe et al., 1983; Gusberti, syed \& Lang, 1988; McCulloch et al. 1990; Kulkarini et al., 1991).

Golub et al. (1987) demonstrated that tetracyclines inhibit MMPs activity by a mechanism independent of their antimicrobial activity. It was shown that the systemically administrated tetracycline could inhibit collagenases derived from inflammatory sources but inhibition of fibroblast collagenase activity needed higher concentrations than could be achieved by this method of delivery (Golub et al., 1985a, 1987b, 1990, 1991, 1992; Lee et al., 1991). Therefore, it is supposed that locally delivered tetracyclines can inhibit both collagenase origins. Metronidazole appears to reduce the prevalence of Porphyromanas gingivalis and spirochetes at active sites in recurrent periodontitis subjects (Loesche et al., 1984). In addition, the results of Teng et al. (1992) suggest that metronidazole treatment can cause the reduction of both active and latent forms of gelatinase. The reduction of gelatinase activity is best explained by the antimicrobial effect of metronidazole which appears to blunt destructive host immune and inflammatory responses. 
Our observation that the mean level of SL decreased after antibiotic treatment could be explained by the fact that local delivery antibiotics plus scaling and root planing may change the microbial flora. This may indirectly or directly cause a larger decrease of MMPs than scaling and root planing alone. It also confirmed that the use of antimicrobial agents as a supplementary treatment increases this effect. However, the greatest decrease was observed in the sites treated by tetracycline or minocycline delivery systems. Previous studies have also shown that tetracyclines, in addition to decreasing the level of microbial periodontal pathogenic bacteria, resulting in a decrease of MMP activity in the gingiva, could decrease the synthesis of pro-MMPs (Rifkin, Vernillo \& Golub, 1993; Uitto et al., 1994) and also protect the endogenous MMP inhibitors (TIMP-1) (Sorsa et al., 1993) and other proteinase inhibitors ( $\alpha 1$-antitrypsin) (Sorsa et al., 1993) from degradation and inactivation by direct and indirect mechanisms. However, it can not be established from this study whether the effect of the tetracyclines on the level of SL is direct, or indirect through the reduction in microbial challenge. This reduction could result in reduction in the microbial proteases and inflammatory mediators such as cytokines, both of which could reduce the production of human MMP. However, the results of the present study demonstrate the effectiveness of antibiotic therapy in reducing crevicular fluid SL level, but it 
cannot be established from this study whether the effect of the antibiotic on the level of SL is direct, or indirect through the reduction in inflammation. This reduction in inflammation could be a consequence of a reduction in antibiotic-sensitive bacteria or the antibiotics could also have a direct, or indirect effect on cells that produce SL. In addition, the use of ELISA methodology, for the quantification of SL in GCF could be criticised for its inability to distinguish between active and latent forms of SL because it cannot be proved from this study whether the effect of the tetracyclines on the level of SL is direct, or indirect through the reduction in active or latent SL.

After treatment, the level of TIMP increased. This might be due to the tendency of free TIMP to bind to active collagenase, SL, and gelatinase (Cawston et al., 1983; Murphy et al., 1989; Howard et al., 1991). The elevation of GCF TIMP was more elevated in the metronidazole group than in the other groups. This indicates that the regulation of TIMP is not solely dependent on the MMPs. The level of GCF TIMP was significantly elevated in the patients who were treated with antimicrobial agents. However, it did not significantly change in the scaling group. It could be speculated, that the extent of healing in the group of patients who received local delivery antibiotics was greater than the group who only received 
scaling. It has been suggested that MMP and TIMP expression can be independently regulated (Overall, 1994).

4.6 .4

In assessing the potential importance of the associations of SL and clinical parameters spearman rank correlation coefficients was performed. It was found that the correlation coefficients between pocket depth and biochemical parameters were weak at baseline, whereas the GCF volume correlation with biochemical parameters were rather strong. At the reassessment visit, the correlations of biochemical parameters and pocket depth and GCF volume were also weak, whereas the correlations in both baseline and reassessment visits were positive. The positive correlation between GCF volume and TIMP was unexpectedly positive which can be due to the local production of TIMP. Since the MGI and PI were categorical data the correlation coefficient were not performed.

The large inter site variation in the levels of SL and TIMP observed in this study may, in part, be responsible for the lack of correlation between proteins and clinical parameters. Further studies are required to determine 
whether variation in the amount of SL among sites is actually linked to changes of disease activity. The weak correlation of the GCF biochemical parameters studied and pocket depth were consistent with the results of Haerian et al. (1995b). It may be, that this association was the result of probing depth measurements taken at one point in time being indicative of a past history of periodontal disease and not necessarily reflecting active periodontal destruction.

There is disagreement in the literature about correlation between clinical parameters and biochemical parameters in gingival tissue fluid. Kryshtalskyj, Sodek and Ferrier (1986), using an experimental dog gingivitis/periodontitis model, have shown that an association between the amount of collagenase activity and GI, PI, changes of attachment level and showed the collagenolytic activities in periodontitis sites fluctuated with time, suggesting a cyclical pattern.

Villela et al. (1987) demonstrated that the correlation between $\mathrm{PD}$ and collagenase activity which were measured in the same technique in three different group of periodontal diseases were different. They found strong correlations between pocket depth and collagenase activity on chronic adult periodontitis sites, partial correlation in localised juvenile periodontitis sites and weak correlation in 
gingivitis sites. Villela et al. (1987) also showed partial and strong correlation between gingival index score and collagenase activity in localised juvenile periodontitis sites and chronic adult periodontitis sites respectively, and failed to show any correlation between collagenase activity and fluid volume. Teng, Sodek and McColloch, (1992) failed to find any correlation between clinical parameters and collagenase activity. Gustafsson et al. (1992) demonstrated that elastase activity in GCF showed a moderate correlation with attachment loss and PD, whereas the enzyme level did not show any correlation with these clinical parameters. Adonogianaki et al. (1993) showed that lactoferrin absolute amounts in GCF and gingival crevicular washings showed rather strong correlations with MGI, PD and GCF volume.

Therefore, it can be inferred that the variation between biochemical correlation and clinical parameters may be associated with different disease status, local host response, periods of rapid connective tissue breakdown, activities of enzymes at the time of sampling, different laboratory techniques of measuring, as well as different methods of GCF sampling. Moreover, the lack of statistical analysis always should be considered in terms of outlying data which could cause a false positive correlation. 
Comparison of stromelysin and tissue inhibitor of metalloproteinase (TIMP) in sites which did or did not respond to treatment

It is supposed that the level of SL and TIMP in sites which failed to respond to treatment showed a different pattern of change to those which responded to treatment. However, our results did not show this pattern and are consistent with the findings of Haerian (1994). The following explanation could be provided to explain this observation: a) treatments resulted in a reduction in the bacterial deposits and removal of the inflamed gingival tissues in both groups of the sites as well as an alteration in the composition, b) since the antibodies in our study, detected total enzyme (latent and active), active enzyme might have been lower in sites which gained attachment or had shallow pockets than those that showed no attachment gain or had deep pockets, as demonstrated by Suomalainen et al. (1992); c) although these sites had significant improvement in clinical conditions, they had no significant attachment gain after treatment; d) the pattern that was observed might have been caused by different level of treatment. 
The clinical and biochemical parameters of the best responding sites (BRS) and worst responding sites (WRS) are discussed separately in order to represent two extremes of response. These sites were analysed regardless of type of treatment. Comparison between changes of GCF SL and TIMP and GCF volume between BRS and WRS revealed that the changes in the level of SL were higher in BRS compare to WRS. Thus, it can be concluded that the reduction of the enzymes is higher in sites which show clinical improvement.

$4 \cdot 6 \cdot 6.1$

\author{
Prediction of treatment response: Using the \\ pocket depth and the state of bleeding on \\ probing
}

Pocket depth and the state of bleeding on probing, are assessed in routine clinical practice to determine the response to treatment, thus sites with $\mathrm{PD}<3.5$ and no $\mathrm{BOP}$ were considered as responding sites. Vanooteghem et al. (1990) reported the prediction of the treatment outcome, using baseline subjective criteria. He showed a maximum $30 \%$ diagnostic accuracy for subjective clinical parameters. Our results showed that pre treatment levels of SL and TIMP did not to distinguish significantly between the best responding and worst responding sites. Moreover, the 
baseline levels of SL were lower in responding sites than was expected.

However, the inability to predict treatment outcome by PD and BOP led us to use the gain in attachment level as an indicator of response to treatment.

$4 \cdot 6 \cdot 6.2$

Prediction of treatment response: Using the attachment level changes as criteria

Dủe to the chronic and episodic nature of periodontitis and the wide range of disease severity affecting different teeth within the same subject, clinical parameters show insufficient ability to predict disease progression (Haffajee et al., 1983a; Jenkins et al., 1988; Kaldahl et al., 1990; Badersten, Nilvèus \& Egelberg, 1990). Investigations have been carried out to assess the association between attachment loss and biochemical components of GCF in order to predict future disease activity (Lamster, et al., 1988b; Persson, De Rouen \& Page, 1990). Our results showed that sites which gained clinical attachment after therapy had a smaller level of SL at baseline compared with sites which lost attachment after therapy. However, our results indicated that SL had a limited value as a diagnostic marker to differentiate between these two clinical categories of sites at a 
statistically significant level. This is in agreement with the study of Haerian (1994).

\subsection{Further study}

A recent paper (Golub et al., 1995) reported that doxycycline was able to inhibit gelatinase extracted from human PMNs whereas the activity of cultured human fibroblasts was resistant to this effect until high concentrations of the drug were used. The results from our study have demonstrated that tetracyclines are able to decrease the level of FIB-SL significantly. Since the ELISA methodology is unable to distinguish between active and latent forms of SL, it can not be established from this study whether the effect of tetracyclines on the level of SL is on active or latent SL. Therefore, it would be worth investigating the effects of locally delivered tetracyclines (tetracycline fibre \& minocycline gel) on GCF MMPs activity using SDS-Page flourography.

Our results showed a tendency toward lower levels of SL in best responder sites, although this association was not strong. Perhaps a study with a larger number of subjects and sites will show a stronger association between clinical and biochemical parameters. Moreover, by using a longer period of follow up the clinical response could be 
evaluated more realistically, thus, increasing the power of statistical tests.

Recent studies demonstrated that the neutrophil collagenases have been identified as the major source of collagenases in adult periodontitis (Sorsa et al., 1988, 1990, Uitto, Suomalainen \& Sorsa, 1990). Our observations showed that the detection of FIB-SL in recurrent periodontitis sites in maintenance patient was low. This tends to confirm the results of the study by Haerian (1994). Therefore, it would be worth while examining the level of these enzymes in the different types of periodontal disease.

Sorsa et al. (1995) showed that the level of MMP-1 was significantly higher in LJP patients' GCF in comparison to AP patients' GCF. Fibroblast type collagenases have been shown to be resistant to tetracycline inhibition after systemic administration. Therefore, it would be of interest to study the efficacy of locally delivered tetracyclines as an adjunctive treatment in the control of periodontal tissue destruction. 


\section{REFERENCES}


Aass, A.M., Tollefsen, T. \& Gjermo, P. (1994) A cohort study of radiographic alveolar bone loss during adolescence. Journal of Clinical Periodontology, 21, 133-138.

Abdellatif, H.M. \& Burt, B.A. (1987) An epidemiological investigation into the relative importance of age and oral hygiene status as determinants of periodontitis. Journal of Dental Research, 66,1318.

Addy, M., Langeroudi, M. \& Hassan, H. (1985) The development and clinical use of acrylic strips containing anti-microbial agents in the management of chronic periodontitis. International Dental Journal, 35, 124-132.

Addy, M., Hassan, H., Moran, J., Wade, W. \& Newcombe, R. (1988) Use of antimicrobial containing acrylic strips in the treatment of chronic periodontal disease. A three-month follow-up study. Journal of Periodontology, 59, 557-564.

Adonogianaki, E., Mooney, J. \& Kinane, D.F. (1992) The ability of gingival crevicular fluid acute phase proteins to distinguish healthy, gingivitis and periodontitis sites. Journal of Clinical Periodontology, 19, 98-102.

Adonogianaki, E., Moughal, N.A. \& Kinane, D.F. (1993) Lactoferrin in the gingival crevice as a marker of polymorphonuclear leucocytes in periodontal diseases. Journal of Clinical Periodontology, 20, 26-31.

Ainamo, J., Lie, T., Ellingsen, B.H., Hansen, B.F., Johansson, L.A., Karring, T., Kisch, J., Paunio, K. \& Stoltze, K. (1992) Clinical responses to subgingival application of a metronidazole $25 \% \mathrm{gel}$ compared to the effect of subgingival scaling in adult periodontitis. Journal of Clinical Periodontology, 19, 723-729. 
Albandar, J.M., Rise, J., Gjermo, P. \& Johansen, J.R. (1986) Radiographic quantification of alveolar bone level changes. A 2-year longitudinal study in man. Journal of clinical Periodontology, 13, 195-200.

Anusaksathien, O., Dolby, A.E. (1991) Autoimmunity in periodontal disease. Journal of oral pathology and Medicine, 20, 101-107.

Armitage, G.C., Dickinson, W.R., Jenderseck, R.S., Levine S.M. \& Chambers, D.W. (1982) Relationship between the percentage of subgingival spirochetes and the severity of periodontal disease. Journal of Periodontology, 53, 550-556.

Aziz-Gandour, I.A. \& Newman, H.N. (1986) The effects of a simplified oral hygiene regime plus supragingival irrigation with chlorhexidine or metronidazole on chronic inflammatory periodontal disease. Journal of Clinical Periodontology, 13, 228-236.

Baab, D.A. \& Oberg, P.A. (1987) The effect of cigarette smoking on gingival blood flow in humans. Journal of Clinical Periodontology, 14, 418-424.

Badersten, A., Nilvèus, R. \& Egelberg, J. (1990) Scores of plaque, bleeding, suppuration and probing depth to predict probing attachment loss. Journal of Clinical Periodontology, 17, 102-107.

Baker, P.J, Evans, R.T, Coburn, R.A \& Genco, R.J (1983) Tetracycline and its derivatives strongly bind to and are released from the tooth surface in an active form. Journal of Periodontology, 54, 580585 .

Binder, T.A., Goodson, J.M. \& Socransky, S.S. (1987) Gingival fluid levels of acid and alkaline phosphatase. Journal of Periodontal Research, 22, 14-19.

Birkedal-Hansen, H., Taylor, R.E., Zambon, J.J., Barwa, P.K. \& Neiders, M.E. (1988) Characterization of collagenolytic activity from strains of Bacteroides gingivalis. Journal of Periodontal Research, 23, 258-264. 
Birkedal-Hansen, H. (1993) Role of Matrix Metalloproteinases in Human Periodontal Diseases. Journal of Periodontology, 64, 474-484.

Birkedal-Hansen, H., Moore, W.G.I., Bodden, M.K., Windsor, L.J., Birkedal-Hansen, B., DeCarlo, A. \& Engler, J.A. (1993) Matrix Metalloproteinases: A Review. Critical Review in Oral Biology and Medicine, 4, 197-250.

Black, A.D. (1918) Roentgenographic studies of tissues involved in chronic mouth infections. American Medical Association Journal, (Dental Summery), 38, 924-929.

Bodden, M.K., Windsor, L.J., Catrina, N.C.M., Yermovsky, A., Brikedal-Hansen, B., Galazka, G., Engler, J. A.\& Birkidal-Hansen, H. (1994) Analysis of the TIMP-1/FIB-CL Complex. Annals of the NewYork Academy of Sciences, 732, 84-95.

Brayer, W.K., Mellonig, J.T., Dunlap, R.M., Marinak, K.W. \& Carson, R.E. (1989) Scaling and root planing effectiveness: The effect of root surface access and operator experience. Journal of Periodontology, 60, 67-72.

Brill, N. (1962) The gingival pocket fluid. Studies of its occurrence, composition and effect. Acta Odontologica Scandinavica, 20, suppl. 32, 1-115.

Brinckerhoff, C.E., McMillan, R.M., Fahey, J.V. \& Harris, E.D. (1979) Collagenase production by synovial fibroblasts treated with phorbol myristate acetate. Arthritis and Rheumatism, 22, 1109-1116.

Britt, M.R., Pohlod, D.J. (1986) Serum and crevicular fluid concentrations after a single oral dose of metronidazole. Journal of Periodontology, 57, 104107.

Brown, R.A. \& Swanson Beck, J. (1989) Statistics on microcomputers. A non-algebric guide to the appropriate use of statistics packages in biomedical research and pathology labroratory practice. 3 Analysis of variance and distributionfree methods. Journal of Pathology, 42, 1256-1262. 
Bunning, R.A., Murphy, G., Kumar, S., Phillips, P.\& Reyolds, J.J. (1984) Metalloproteinase inhibitors from bovine cartilage and body fluids. European Journal of Biochemistry, 139, 75-80.

Burns, F.R., Stack, M.S., Gray, R.D. \& Patersom, C.A. (1989) Inhibition of purified collagenase from alkalineburned rabbit corneas. Investigations into Opthalmology Review, 42, 707-724.

Carpenter, A.B. (1992) Enzyme-linked immuonoassays. In:Manual of Clinical laboratory Immuonology, 4 th ed. pp. 2-9.

Cawston, T.E., Murphy, G., Mercer, E., Calloway, W.A., Hazleman, B.L \& Reynolds, J.J. (1983) The interaction of purified rabbit bone collagenase with purified rabbit bone metalloproteinase inhibitions. Biochemical Journal, 211, 313-318.

Cawston, T.E. \& Mercer, E. (1986) Preferential binding of collagenase to $\alpha 2$-Macroglobulin in the presence of tissue inhibitor of metalloproteinases. FEBS Letters, 209, 9-12.

Cimasoni, G., Ishikawa, I. \& Jaccard, F. (1977) In Borderland between Caries and Periodontal Disease (T. Lehner, ed.), pp. 13-41, Academic Press, London.

Chang, C., Houck, J.C. (1970) Demonestration of the chemotactic properties of collagen. Proceeding of the society for Experimental Biology and Medicine, $134,22-26$.

Chopra, I. \& Howe T.G. (1978) Bacterial resistance to the tetracyclines. Microbiology Review, 42, 707-724.

Cooksley, S., Hipkiss, J.B., Tickle, S.P., Holmes-Ievers, E., Docherty, A.J., Murphy, G. \& Lawson, A.D. (1990) Immunoassay for the detection of human collagenase, stromelysin, tissue inhibitor of metalloproteinases (TIMP) and enzyme-inhibitor complexes. Matrix, 10 (5), 285-291. 
Curtis, M.A., Griffiths, G.S, Price, S.J., Coulthurst, S.K. \& Johnson, N.W. (1988) The total protein concentration of gingival crevicular fluid. Variation with sampling time and gingival inflammation. Journal of clinical Periodontology, $15,628-632$.

Davies, A.H., MacFadzen, J.A., Squires, S. (1964) Treatment of Vincent's stomatitis with metronidazole. British Medical Journal, 1, 11491150 .

Ding, Y., Liede, K., Ingman, T., Sepper, R., Konttinen, Y. T. \& Sorsa, T. (1994) Gingival crevicular fluid and salivary matrix metalloproteinases of heavy smokers as indicators of periodontal health. Annals of the NewYork Academy of Sciences, 732, $453-455$.

Docherty, A.J.P. \& Murphy, G. (1990) The tissue metalloproteinase family and the inhibitor TIMP: A study using DNAs and recombinant proteins, Annales of the Rheumatic Disease, 49, 469-479.

Drouin, , L., Overall, C.M., \& Sodek, J. Identification of matrix metalloproteinase inhibitor (TIMP) in human parotid and submandibular saliva: Partial purification and characterization. Journal of Periodontal Research, 23, 370-377.

Duggar, B.M. (1948) Aureomycib: a product of the continuing search for new antibiotics. Annuals of the NewYork Academy of Science, 51, 177-181.

Eckles, T.A., Reinhardt, R.A., Dyer, J.K., Tussing, G.J., Szydlowski, W.M. \& DuBous, L.M. (1990) Intracrevicular application of teracycline in white petrolatum for the treatment of periodontal disease. Journal of Clinical Periodontology, 17, $454-562$.

Edwards, D.R., Murphy, G., Reynolds, J.J., Whitham, S.E., Docherty, A.J.P., \& Angel, P. (1987) Transforming growth factor beta modulates the expression of collagenase and metalloproteinase inhibitor. EMBO journal, 6, 1899-1904. 
Eisenhauer, D.A., Hutchinson, R., Javed, T. \& McDonald, J.K. (1983) Identification of a cathepsin B-like protease in the crevicular fluid of gingivitis patients. Journal of Dental Research, 62, 917-921.

Egelberg, J. \& Attstrom, R. (1973) Comparison between orifice and intracrevicular methods of sampling gingival fluid. Journal of Periodontal Research, $8,384-388$.

El-Attar, T.M.A., Lin, H.S. \& Shultz, R. (1988) Effect of minocycline on prostaglandin formation in gingival fibroblasts. Journal of Periodontal Research, 23, $285-286$.

Eliasson, S., Lavstedt, S. \& Ljungheimer, C. (1986) Radiographic study of alveolar bone height related to tooth and root length. Community Dental Oral Epidemiology, 14, 169-171.

Fiehn, N.E. (1990) Therapeutic use of antibiotics in dentistry. Journal of Cinical Periodontology, 17, 528-529.

Fleischer, H.C., Mellonig, J.T., Brayer, W.K., Gray, J.L. \& Barnett, J.D. (1989) Scaling and root planing efficacy in multirooted teeth. Journal of Periodontology, 60, 402-409.

Friedman, M. \& Golomb, G. (1982) New sustained release dosage form of chlorhexidine for dental use. I. Development and kinetics of release. Journal of Periodontal Research, 17, 323-328.

Gabler, W.L. \& Creamer, H.R. (1991) Suppression of human neutrophil functions by tetracycline. Journal of Periodontal Research, 26, 52-58.

Genco, R.J. (1981) Antibiotics in the treatment of human periodontal disease. Journal of Periodontology, 52, 545-558.

Genco, R.J., Evans, R.T.; Ellison, S.A. (1969) Dental research in micro-biology with emphasis on periodontal disease. Journal of American Dental Association, 78, 1016-1036. 
Gibbs, C.H., Hirschfeld, J.W., Lee, J.G., Low, S.B., Magnusson, I., Thousand, R.R., Yerneni, P. \& Clark, W.B. (1988) Description and clinical evaluation of a new computerized periodontal probe- the Florida Probe. Journal of Clinical Periodontology, 15, 137144 .

Goadby, K.W. (1907) Erasmus Wilson's lecture on pyorrhea alveolaris. Lancet i:633.

Goldberg, G.I., Frisch, S.M., He, C., Wilhelm, S.M., Reich, R., \& Collier, I.E. (1990) secreted prteases. Regulation of their activity and their possible role in metastases. Annals of the NewYork Academy of Sciences, 580, 375-384.

Golub, L.M., Lee, H-M., Lehrer, G., Nemiroff, A., McNamara, T.F., Kaplan, R. \& Ramamurthy, N.S. (1983) Minocycline reduces gingival collageolytic activity during diabetes: Preliminary observation and a proposed new mechanism of action. Journal of Periodontal Research, 18, 516-526.

Golub, M., Ramamurthy, N., McNamara, T.F., Gomes, B., Wolff, M., Casino, A., Kapoor, A., Zambon, J. Ciancio, S., Schneir, M., \& Perry, H. (1984) Tetracyclines inhibit tissue collagenase activity. A new mechanism in the treatment of periodontal disease. Journal of Periodontal Research, 19, 651655 .

Golub, L.M., Wolff, M., Lee, H.M., McNamara, T.F., Ramamurthy, N.S., Zambom, J. \& Ciancio, S. (1985a) Further evidence that tetracyclines inhibit collagenase activity in human crevicular fluid and from other mammalian sources. Journal of Periodontal Research, 20, 12-23.

Golub, L.M., Goodson, J.M., Lee, H.M., Vidal, A.M., McNamara, T,F. \& Ramamurthy, N.S. (1985b) Tetracyclines inhibit tissue collagenases. Effects of ingested low-dose and local delivery systems. Journal of Periodontology, 56, (Spec. Iss), 93-97. 
Golub, L.M., McNamara, T.F., D'Angelo G., Greenwald, R.A., Ramamurthy, N.S. (1987a) A non-antibacterial chemically-modified tetracycline inhibits mammalian collagenase activity. Journal of Dental Research, $66,1310-1314$.

Golub, L.M., Wolff, M., Vidal, A.M., Ramamurthy, N.S. \& McNamara, T.F. (1987b) Low dose minocycline therapy: effects on crevicular fluid collagenase and subgingival microflora, Journal of Dental Research, 66 (Spec. Iss.), JADR Abstr. no. 384.

Golub, L.M., Ciancio, S., Ramamurthy, N.S., Leung, M., McNamara, T.F. (1990) Low-dose doxycycline therapy Effect on gingival and crevicular fluid collagenase activity in humans. Journal of Periodontal Research, 25, 321-330.

Golub, L.M., Ramamurthy, N.S., McNamara, T.F., Greenwald, R.A., Rifkin, B.R. (1991) Tetracyclines inhibit connective tissue breakdown: new therapeutic implications for an old family of drugs. Critical Reviews in Oral Biology of Medicine, 2, 297-321.

Golub, L.M., Suomalainen, K. \& Sorsa, T. (1992) Host modulation with tetracyclines and their chemically modified analogues. Current Opinion in Dentistry, $2,80-90$.

Golub, L.M., Sorsa, T., Lee, H.M., Ciancio, S., Sorbi, D., Ramamurthy, N.S., Gruber, B., Salo, T., \& Konttinen, Y.T. (1995) Doxacycline inhibits neutrophil (PMN)-type matrix metalloproteinases in human adult Periodontitis gingiva. Journal of clinical Periodontology, 22, 100-109.

Gomes, B.C., Golub, L.M. \& Ramamurthy, N.S. (1984) Tetracyclines inhibit parathyroid hormone induced bone resorption in organ culture. Experientia, 40, $1273-1275$.

Goodson, J.M., Haffajee, A.D. \& Socransky, S.S. (1979) Periodontal therapy by local delivery of tetracycline. Journal of Clinical Periodontology, $6,83-92$. 
Goodson, J.M., Tanner, A.C., Haffajee, A.D., Sornberger, G.C. \& Socransky, S.S. (1982) Patterns of progression and regression of advanced destructive periodontal disease. Journal of clinical Periodontology, 9, 472-481.

Goodson, J.M., Holborow, D., Dunn, R.L., Hogan, P. \& Dunham, S. (1983) Monolithic tetracyclinecontaining fibers for controlled delivery to periodontal pockets. Journal of Periodontology, 54, 575-579.

Goodson, G.M., Offenbacher, S., Farr, D.H. \& Hogan, P.E. (1985a) Periodontal disease treatment by local drug delivery. Journal of Periodontology, 56, 265-272.

Goodson, J.M., Cugini, M.A., Kent, R.L., Armitage, G.C., Cobb, C.M., Fine, D., Fritz, M.E., Green, E., Imoberdorf, M.J., Killoy, W.J. et al. (1991) Multicentre evaluation of tetracycline fiber therapy: clinical response. Journal of Periodontal Research, 26, 371-379.

Gordon, J., Walker, C., Hovliaras, C., \& Socransky, S. (1990) Efficacy of clindamycin hydrochloride in refractory periodontitis: 24 month results. Journal of Periodontology, 61, 686-691.

Greenwald, R.A., Golub, L.M., Lavictes, B., Ramamurthy, N.S., Laskin, R. \& Gruber, B. \& MCNamara T.F. (1987) Tetracyclines inhibit human synovial collagenase in vivio and in vitro. Journal of Rheumatology, 14, 28-32.

Griffiths, G.S., Curtis, M.A. \& Wilton, J.M.A.

Selection of a filter paper with optimum properties for the collection of gingival crevicular fluid. Journal of periodontal Research, 23,33-38.

Griffiths, G.S., Sterne, J.A.C., Wilton, J.M.A., Eaton, K.A. \& Johnson, N.W. (1992) Associations between volume and flow rate of gingival crevicular fluid and clinical assessments of gingival inflammation in a population of British male adolescents. Journal of Clinical Periodontology, 19, 464-470. 
Grossi, S.G., Zambon, J.J., Ho, A.W., Koch, G., Dunford, R.G., Machtei, E.E., Norderyd, O.M. \& Genco, R.J. (1994) Assessment of risk for periodontal disease. I. Risk indicators for attachment loss. Journal of Periodontology, 65, 260-267.

Gusberti, FA., Syed, S.A. \& Lang, N.P. (1988) Combined antibiotic (Metronidazole) and mechanical treatment effects on the subgingival bacterial flora of sites with recurrent periodontal disease. Journal of Clinical Periodontology, 15 (6), 353-359.

Gustafsson, A., Asman, B. \& Bergstrom, K., \& Soder P-O. (1992) Granulocyte elastase in gingival crevicular fluid. A possible discriminator between gingivitis and periodontitis. Journal of Clinical Periodontology, 19, 535-540.

Haerian, A. (1994) Gingival crevicular matrix metalloproteinases and their inhibitor in health, disease and treated periodontitis. Ph.D thesis.

Haerian, A., Adonogianaki, E., Mooney, J., Doucherty, J. P., \& Kinane, D.F. (1995a) Gingival crevicular stromelysin, collagenase and tissue inhibitor of metalloproteinases levels in healthy and diseased sites. Journal of Clinical Periodontology, 22, 505-509.

Haerian, A., Adonogianaki, E., Mooney, J., Manos, A. \& Kinane, D.F. (1995b) Effect of treatment on gingival crevicular collagenase, stromelysin and tissue inhibitor of metalloproteinases and their ability to predict response to treatment. Journal of Clinical Periodontology, In press.

Haffajee, A.D., Socransky, S.S. \& Goodson, J.M. (1983a) Clinical parameters as predictors of destructive periodontal disease activity. Journal of Clinical Periodontology, 10, 257-265.

Haffajee A.D., Socransky S.S. (1994) Microbial eatiological agents of destructive periodontal disease. Periodontology 2000, 5, 78-111. 
Hakkarainen, K., Uitto, V.J. \& Ainamo, J. (1988) Collagenase activity and protein content of sulcular fluid after scaling and occlusal adjustment of teeth with deep periodontal pockets. Journal of Periodontal Research, 23, 204-210.

Harris, E.D., Jr. \& Vater, C.A. (1982) Vertebrate collagenases. Methods Enzymology, 82, 423-452.

Hasty, K.A., Hibbs, M.S., Kang, A.H. \& Mainardi, C.L. (1984) Heterogenety among human collagenases demonestrated by monoclonal antibody that selectively recognizes and inhibits human neutrophil collagenase. Journal of Experimental Medicine, 159, 1455-1463.

Higashi, K., Morisaki, K., Hayashi, S., Kitamura, M., Fujimoto N, Kimura S, Ebisu S, Okada H. (1990) Local ofloxacin delivery using a controlled-release insert (PT-01) in the human periodontal pocket. Journal of Periodontal Research, 25, 1-5.

Hirsch H.z., Tarkowski, A., Miller, E.J. Gay S., Koopman, W.J. \& Mestecky, J. (1988) Autoimmunity to collagen in adult periodontal disease. Journal of Oral Pathology, 17, 456-459.

Hitzig C., Charbit, Y., Bitton, C., Fosse, T., Teboul, M., Hannoun, L. \& Varonne, R. (1994) Topic metronidazole as an adjunct to subgingival debridment in the treatment of chronic periodontitis. Journal of Clinical Periodontology, 21, 146-151.

Howard, E.W., Bullen, E.C. \& Banda, M.J. Preferential inhibition of 72 and $92-\mathrm{KDa}$ gelatinases by tissue inhibitor of Metalloproteinases.2. Journal of Biological Chemistry, 266, 13070-13075.

Hughes, J.T., Rozier, R.G. \& Ramsey, D.L. (1982) Natural history of dental diseases in north carolina. Durham, Carolina Academic Press. 
Ingman, T., Sorsa, T., Suomalainen, K., Halinen, S., Lindy, O., Lauhio, A., Saari, H., Konttinen, Y.T. \& Golub, L. (1993) Tetracycline inhibition and the cellular source of collagenase in gingival crevicular fluid in different periodontal disease. A review article. Journal of Periodontology, 64, 82-88.

Ingman, T., Sorsa, T., Michaelis, J. \& Konttinen, Y.T. (1994a) Matrix metalloproteinases-1, $-3,-8$ in adult periodontitis in situ. Annals of the NewYork Academy of Sciences, 732, 459-461.

Ingman, T., Sorsa, T., Michaelis, J., Konttinen, Y.T. (1994b) Immunohistochemical study of neutrophil- and fibroblast-type collagenase and stromelysin-1 in adult periodontitis. Scandinavian Journal of Dental Research, 102, 342-349.

Ingman, T., Tervahartiala, T., Ding, Y., Tschesche, H., Haerian, A., Kinane, D., Konttinen, Y.T., Sorsa, T. (1995) The levels of matrix metalloproteinases (MMP$1,-3,-8$ and -9$)$ and tissue inhibitor of matrix metalloproteinases (TIMP-1) in gingival crevicular fluid and saliva of periodontitis patients. Submitted in Journal of Clinical Periodontology.

Isidor, F., Karring, T. \& Attstrom R.

Reproducibility of pocket depth and attachment level measurements when using a flexible splint. Journal of Clinical Periodontology, 11, 662-668.

Ismail, A.I., Morrison, E.C., Burt, B.A., Caffesse, R.G., Kavanagh, M.T. (1990) Natural history of periodontal disease in adults: findings from the tecumseh periodontal disease study. Journal of Dental Research, 69 (2),430-435.

Jenkins, W.M.M., MacFarlane, T.W. \& Gilmour, W.H. Longitudinal study of untreated periodontitis: (I). Clinical findings. Journal of Clinical Periodontology, 15, 324-330.

Jenkins, W.M.M., MacFarlane, T.W., Gilmour, W.H., Ramsay, I. \& Mackenzie, D. (1989) Systemic metronidazole in the treatment of periodontitis. Journal of Clinical Periodontology, 16, 443-450. 
Kaldahl, W.B., Kalkwarf, K.L., Patil, K.D. \& Molvar, M.P. (1990) Relationship of gingival bleeding, gingival suppuration, and supragingival plaque to attachment loss. Journal of Periodontology, 61, 347-351.

Khoo, J.G.L. \& Newman, H.N. (1983) Subgingival plaque controle by a simplified oral hygine regime plus local chlorhexidine or metronidazole. Journal of Periodontal Research, 18, 607-619.

Kinane, D.F. \& Davies, R.M. (1990) Periodontal manifestations of systemic disease. In Oral manifestations of systemic disease, pp 512-536, eds Jones J.H and Mason D.K., London: Baillière-Tindall.

Kinane, D.F. (1992) Metalloproteinases in the pathogenesis of periodontal disease. Current Science, 25-32.

Klinge, B., Kuvatanasuhati, J., Attstrom, R., Kalfas, S., \& Edwardsson, S. (1992) The effect of topical metronidazole therapy on experimentally-induced periodontitis in the beagle dog. Journal of Clinical Periodontology, 19, 702-707.

Kornman, K.S., Newman, M.G., Fleming, T., Alvarado, R., Nachnani, S. (1989) Treatment of refractory periodontitis with metronidazole plus amoxicillin or augmentin. Journal of Dental Research, 68 (Spec. Issue), 917 (Abstr. 403).

Kornman, K.S. (1993) Controlled-release local delivery antimicrobials in periodontics: prospects for the future. Journal of Periodontology, 64, 782-791.

Krane, S.M. (1994) Clinical importance of metalloproteinases and their inhibitors. Annals New York Academy of Sciences, 732, 1-10.

Kryshtalskyj, E., Sodek, J. \& Ferrier, M. (1986) Correlation of collagenolytic enzymes and inhibitors in gingival crevicular fluid with clinical and microscopic changes in experimental periodontitis in the dog. Archives of oral biology, 31 (1), 21-31. 
Kryshtalskyi, E. \& Sodek, J. (1987) Nature of collagenolytic enzyme and inhibitor activities in crevicular fluid from healthy and inflamed periodontal tissues of beagle dogs. Journal of Periodontal Research, 22, 364-269.

Kulkarni, G.V., Lee, W.K., Aitken, S., Birek, P., McCulloch, C.A. (1991) A randomised, placebocontrolled trial of doxycycline; effect on the microflora of recurrent periodontitis lesions in high risk patients. Journal of Periodontology, 62, 197-202.

Lamster, I.B., Vogel, R.I., Hartley, L.J., DeGeorge, C.A. \& Gordon, J.M. (1985) Lactate dehydrogenase. Bglucuronidase and arylsulfatase activity in gingival crevicular fluid associated with experimental gingivitis in man. Journal of Periodontology, 56, 139-147.

Lamster, I.B., Oshrain, R.L. \& Gordon, J.M. (1986) Enzyme activity in human gingival crevicular fluid: considerations in data reporting based on analysis of individual crevicular sites. Journal of Clinical Periodontology, 13, 799-804.

Lamster, I.B., Oshrain, R.L., Florello, L.A., Celenti, R.S. \& Gordon, J.M. (1988a) A comparison of 4 methods of data presentation for lysosomal enzyme activity in gingival crevicular fluid. Journal of Clinical Periodontology, 15, 347-352.

Lamster, I.B., Oshrain, R.L., Harper, D.S., Celenti, R.S., Hovliaras, C.A. \& Gordon, J.M. (1988b) Enzyme activity in crevicular fluid for detection and prediction of clinical attachment loss in patients with chronic adult periodontitis. Six month results. Journal of Periodontology, 59, 516-523.

Lamster, I.B., Harper, D.S., Goldstein, S., Celenti, R.S. \& Oshrain, R.L. (1989) The effect of sequential sampling on crevicular fluid volume and enzyme activity. Journal of Clinical Periodontology, 16, $252-258$. 
Larivèe, L., Sodek, J. \& Ferrier, J.M.

Collagenase and collagenase inhibitor activities in crevicular fluid of patients receiving treatment for localised juvenile periodontitis. Journal of Periodontal Research, 21, 702-715.

Lauhio, A., Sorsa, T., Lindy, O., Suomalainen, K., Saari, H., Golub, L.M. \& Konttinen, Y.T. (1992) The anticollagenolytic potential of lymecycline in the long-term treatment of reactive arthritis. Arthritis Rheumatology, 35, 195-198.

Lee, W., Aitken, S., Kulkarni, G., Birek, P., Overall, C. M., Sodek, J. \& McCulloch, C.A. (1991) Collagenase activity in recurrent periodontitis: relationship to disease progression and doxacycline therapy. Journal of Periodontal Research, 26 (6), 479-485.

Lekovic V., Kenney E.B., Carranza F.A. \& Endress B.

The effect of metronidazole on human periodontal disease. A clinical and bacteriological study. Journal of Periodontology, 54, 476-480.

Lindhe, J., Heijl, L., Goodson, J.M. \& Socransky, S.S. (1979) Local tetracycline delivery using hollow fiber devices in periodontal therapy. Journal of clinical Periodontology, 6, 141-149.

Lindhe J., Liljenberg B., Adielson, B., Borjesson, I. (1982) The effect of metronidazole therapy on human periodontal disease. Journal of Periodontal Research, 17 (5), 534-536.

Lindhe J., Lilgenberg B., Adielson, B. \& borjesson, I. (1983) Use of metronidazole as a probe in the study of human periodontal disease. Journal of Clinical Periodontology, 10, 100-112.

Lindhe, J., Liljenberg, B. \& Adielson, B. (1983) Effect of long-term tetracycline therapy on human periodontal disease. Journal of Clinical periodontology, 10, 590-601.

Lindhe, J. (1989) Textbook of Clinical Periodontology, 2nd ed, p 19, Copenhagen: Munskgaard. 
Lobene, R.R., Weatherford, T., Ross, N.M., Lamm, R.A., \& Menaker, L. (1986) A modified gingival index for use in clinical trials. Clinical Preventive Dentistry, 8, 3-6.

Löe, H. \& Holm-Pedersen, P. (1965) Absence and presence of fluid from normal and inflamed gingivae. Periodontics, 3, 171-177.

Löe, H. \& Silness, J. (1963) Periodontal disease in pregnancy.I. Prevalence and severity. Acta odontologica Scandinavica, 21, 533-551.

Loesche, W.J. (1976) Chemotherapy of dental plaque infections. Oral Science Review, 9, 65-107.

Loesche, W.J., Syed, S.A., Morrison, E.C., Laughon, B., Grossman, N.S. (1981) Treatment of Periodontal infections due to anaerobic bacteria with shortterm treatment with metronidazole. Journal of Clinical Periodontology, 8 (1), 29-44.

Loesche, W.J., syed, S.A., Morrision, E.O., Kerry, G.A., Higgins, T. \& Stoll, J. (1984) Metronidazole in periodontitis I. clinical and bacteriological results after 15 to 30 weeks. Journal of Periodontology, 55, 325-335.

Loesche W.J., Schmidt, E., Smith, A., Schwarcz, J. \& Smith, A. (1991) Effects of metronidazole on periodontal treatment needs. Journal of Periodontology, 62, 247-257.

Loesche, W.J., Giordano J.R., Hujoel P., Schwarcz, J., Smith, B.A. (1992) Metronidazole in periodontitis: Reduced need for surgery. Journal of Clinical Periodontology, 19, 103-112.

Loos, B., Nylund, K., Claffey, N. \& Egelberg, J. (1989) Clinical effects of root debridement in molar and non-molar teeth. A 2-year follow-up. Journal of Clinical Periodontology, 16, 498-504.

Lovejoy, B., Cleasby, A., Lambert M.H, Hassell, A.M., Luther, M.A., Weigl, D., McGeehan, G. \& Jordan, S. R. (1994) Annals of the NewYork Academy of Sciences, 375-378. 
Lundström, A., Johansson, L.A. \& Hamp, S.E. (1984) Effect of combined systemic antimicrobial therapy and mechanical plaque control in patients with recurrent periodontal disease. Journal of Clinical Periodontology, 11, 321-330.

MacAlpine, R., Magnusson, I., Kiger, R., Cigger, M., Garrett, S. \& Egelberg, J. (1985) Antimicrobial irrigation of deep pockets to supplement oral hygiene instruction and root debridement. I. Biweekly irrigation. Journal of Clinical Periodontology, 12, 568-577.

Maehara, R., Hinode D., Terai, H., Sato, M., Nakamura, R., Matsuda, N., Tanaka, T., Sugihara, K. Inhibition of bacterial and mammalian collagenolytic activities by tetracyclines. Nippon Shishubyo Gakkai Kaishi, 30 182-190.

Magnusson, I, Lindhe J., Yoneyama T. \& Liljenberg B. (1984) Recolonization of a subgingival microbiota following scaling in deep pockets, Journal of Clinical Periodontology, 11, 193-207.

Martin, R.R., Warr, G.A., Couch, R.B., Yeager, H.\& Knight, V. (1974) Effects of tetracycline on leukotaxis. Journal of Infectious Diseases, 129, 110-116.

McCulloch, C.A.G., Birek P., Overall C., Aitken S., Lee W. \& KulKarni G. (1990) Randomized controlled trial of doxycycline in prevention of recurrent periodontitis in high risk patients: antimicrobial activity and collagenase inhibition. Journal of clinical periodontology, 17, 616-622.

Meikle, M.C., Atkinson, S.J., Ward, R.V., Murphy, G. \& Reynolds, J.J. (1989) Gingival fibroblasts degrade type I collagen films when stimulated with tumour necrosis factor and interlukin 1: Evidence that breakdown is mediated by metalloproteinases. Journal of periodontal Research, 24, 207-213. 
Minabe, M., Uematsu, A., Nishijima, K., Tomomatsu E., Tamura $T$, Hori $T$, Umemoto $T$ and Hino $T$. (1989) Application of a local drug delivery system to periodontal therapy. I. Development of collagen preparations with immobilised tetracycline. Journal of Periodontology, 60, 113-117.

Moore, W.E.C. (1987) Microbiology of periodontal disease. Journal of Periodontal Research, 22, 335-341.

Murayama, Y., Nomura, Y. Yamaoda, A., Ueda, M., Hori, T., Minabe, M., Umemoto, T., Ishikawa, I., Uraguchi, R., Ueno, K., Watanabe, K. \& Ogawa, N. (1988a). Local administration of minocycline for periodontitis. Double blind comparative study of LS-007. Journal of Japanese Association of Periodontology, 30, 206-222.

Murphy, G., Cawston, T.E., \& Reynolds, J.J. (1981) An inhibitior of collagenase from human amniotic fluid. Purification, characterization and action on metalloproteinases, Biochemical Journal, 195, 167-170.

Murphy, G., Reynolds, J.J. \& Werb, Z. (1985) Biosynthesis of tissue inhibitor of metalloproteinases by human fibroblasts in culture: Stimulation by 12-0tetradecanoylphorbol 13-acetate and interlukin 1 in parallel with collagenase. Journal of Biological Chemistry, 260, 3079-3083.

Murphy, G., Koklitis, P. \& Carne, A.F. (1989) Dissociation of tissue inhibitor of Metalloproteinases (TIMP) from enzyme complexes yields fully active inhibitor. Biochemical Journal, 261, 1031-1034.

Murphy, G., Allan, J., Willenbrock, F., Cockett, M.I., O'Connell, J.P., \& Docherty, A.J.P. (1992) The role of the C-terminal domain in collagenase and stromelysin specificity, Jounal of Biological Chemistry. 267, 9612-9618.

Murphy, G. \& Docherty, A.J.P. (1992) The matrix metalloproteinases and their inhibitors, American Journal of Respiratory Cell and Molecular Biology, 7. $120-125$. 
Nakashima, K., Demeurisse, C. \& Cimasoni, G. (1994) The recovery efficiency of various materials for sampling enzymes and polymorphonuclear leukocytes from gingival crevices. Journal of Clinical Periodontology, 21, 479-483.

Needleman, I.G., Watts, T.L. (1989) The effect of $1 \%$ metronidazole gel in routine maintenance of peristent furcation involvement in human being. Journal of Periodontology, 60, 699-703.

Newman, H.N., Yeung F.I.S., Wan Yousof, W.Z.A.B., \& Addy, M. (1984) Slow release metronidazole and a simplified mechanical oral hygiene regimen in the control of chronic periodontitis. Journal of Clinical Periodontology, 11, 576-582.

Noguchi, T., Izumizawa, K., Fukuda, M., Kitamura, S., Suzuki, Y., Ikura, H. (1984) New method for local drug delivery using resorbable base material in periodontal therapy. Bull Tokyo Medical Dental University, 31, 145-153.

Nomura, T., Takahashi, T. \& Hara, K. (1993) Expression of TIMP-1, TIMP-2 and collagenase mRNA in periodontitis-affected human gingival tissue. Journal of Periodontal Research, 28, 354-362.

Nordland, P., Garrett, S., Kiger, R., Vanooteghem, R., Hutchens, L. H. \& Egelberg, J. (1987) The effect of plaque control and root debridement in molar teeth. Journal of Clinical Periodontology, 14, $231-236$.

Okada, Y., Watanabe, S., Nakanishi, I., Kishi, J., Hayakawa, T, , Watorek, W., Travis, J., Nagase, H. (1988) Inactivation of tissue inhibitor of metalloproteinases by neutrophil elastase and other serine proteinases. FEBS Letter, 229(1), 157-160.

Okuda, K., Wolff, L., Oliver, R., Osborn, J., Stoltenberg, J., Bereuter, J., Anderson, L., Foster, P., Hardie, N., Aeppli, D., and Hara, K. (1992) Minocycline slow-release formulation effect on subgingival bacteria. Journal of Periodontology, 63,73-79. 
Oppenheim, F.G. (1970) Preliminary observations on the presence and origin of serum albumin in human saliva. Helvetica odontologica Acta, 14, 10-17.

Overall, C.M., Wrana, J.L. \& Sodek, J. (1989) Independent regulation of collagenase, $72 \mathrm{kDa}$ progelatinase and metalloproteinase inhibitor expression in human fibroblasts by transforming growth factor beta. Journal of Biological Chemistry, 264, 1860-1869.

Overall, C.M., Wrana, J.L., \& Sodek, J. (1991) Induction of formative and resorptive cellular phenotypes in human gingival fibroblasts by TGF-beta-1 and concanavalin-A: regulation of matrix metalloproteinases and TIMP. Journal of Periodontal Research, 26, 279-282.

Overall, C.M. (1994) Regulation of tissue inhibitor of matrix metalloproteinase expression. Annals of the NewYork Academy of Sciences, 732, 51-65.

Page R.C. \& Schroeder, H.E. (1976) Pathogenesis of inflammatory periodontal disease. Laboratory Investigation, 34, 235-249.

Palmer, R.M. (1988) Tabacco smoking and oral health. British Dental Journal, 164 (8), 258-260.

Papapanou, P.N., Wennström, J.L. (1990) A 10-year retrospective study of periodontal disease progression. Clinical characteristristics of subjects with pronounced and minimal disease development. Journal of Clinical Periodontology, 17 (2), 78-84 .

Persson, G.R. \& Page, R.C. (1990) Effect of sampling time and repetition on gingival crevicular fluid and aspartate aminotransferase activity. Journal of Periodontal Research, 25, 236-242.

Persson, G.R., DeRouen, T.A. \& Page, R.C. (1990) Relationship between gingival crevicular fluid levels of aspartate aminotransferase and active tissue destruction in treated chronic periodontitis patients. Journal of Periodontal Research, 25, 8187. 
Plaisance K.I., Quintiliani R. \& Nightingale C.H (1988) The pharmacokinetics of metronidazole and its metabolites in critically ill patients. Journal of Antimicrobiology Chemotheraphy, 21, 195-200.

Pruzanski W., Greenwald R.A. Street I.P., Laliberte F., Stefanski E. \& Vadas P. (1992) Inhibition of enzymatic activity of phospholipases A2 by minocycline and doxacycline. Biochemical Pharmacology, 44 (6), 1165-1170.

Puchalsky, C.S., Greeway, D., Grossi, S., Lyon-Bottonfield. E., Huber, L. \& Christersson, L.A. (1988) Topical application of tetracycline-HCL in human Periodontitis. Journal of Dental Research, 67, 208, abstr. 766 .

Ramamurthy, N.S., Vernillo, A.T., Greenwald, R.A., Lee, H. M., Sorsa, T., Golub, L. M. \& Rifkin. B.R. (1993) Reactive oxygen species activate and tetracyclines inhibit rat osteoblast collagenase. Journal of Bone and Mineral Research, 8, 1247-1253.

Rams, T.M., Keyes, P.H. (1983) A rationale for the management of periodontal diseases: effects of tetracycline on subgingival bacteria. Journal of American Dental Association, 107 (1), 37-41.

Ray, H.G. \& B. Orban. (1948) Deep necrotic foci in the gingiva. Journal of Periodontology, 19, 91-97

Rifkin, B.R., Vernillo, A.T. \& Golub, L.M. (1993) Blocking periodontal disease progression by inhibiting tissue-destructive enzymes: a potential therapeutic role for tetracycline and their chemically-modified analogs. Journal of periodontology, 64, (suppl.), 819-827.

Rompen, E.H., Kohl, J., Nusgens, B. \& Lapiere, C.M. (1993) Kinetic aspects of gingival and Periodontal ligament fibroblast attachment to surfaceconditioned dentine. Journal of Dental Research, 72, 607-612. 
Rosania, D.A. \& Kornman, K.S. (1981) The subgingival microflora following root planning with or without local tetracycline application. Journal of Dental Research, 60, 604, abstr. 1179.

Rossomando, E.F, Kennedy, J.E. \& Hadjimichael, J.

Tumour necrosis factor alpha in gingival crevicular fluid as a possible indicator of periodontal disease in humans. Archives of Oral Biology. $35(6), 431-434$.

Saglie, R. \& Elbaz, J.J. (1983) Bacterial penetration into the gingival tissue in periodontal disease. Journal of West Society of Periodontology, 31, 8593.

Sasaki, T., Ramamurthy, N.S., Yu, Z. \& Golub, L.M. (1992a) Tetracycline adminestration increases protein (presumably procollagen) synthesis and secretion in periodontal ligament fibroblasts of streptozotocininduced diabetic rats. Journal of Periodontal Research, 27, 631-639.

Sasaki, T., Ramamurthy, N.S \& Golub, L.M. (1992b) Tetracycline administration increases collagen synthesis in osteoblasts of streptozotocin-induced diabetic rats: a quantitative autoradiographic study. Calcified Tissue International, 50, 411419 .

Schluger, S., Yuodelis, R., Page, R.C., Johnson, R.H. (1990) Periodontal disease. 2nd ed, p 3-4, Lea \& Febiger.

Schenkein, Burmeister, JA, Koertge, TE, Brooks, CN, Best, AM, Moore, L.V. \& Moore, W.E. (1993) The influence of race and gender on periodontal microflora. Journal of Periodontology, 64, 292-296.

Schneir, M., Ramamurthy, N., Golub, L. Minocycline-treatment of diabetic rats normalizes skin collagen production and mass: possible causative mechanisms. Matrix, 10(2), 112-123. 
Schroeder, K., Lee, H., Wolff, M., Ramamurthy, N. \& Golub, L.M. (1990) Low-dose tetracyclines decrease elastase and $\beta$-glucuronidase activities in gingival crevicular fluid. Journal of Dental Research, 69, 245 (Spec. Iss.) Abstr. 1090.

Schwartz, J., Stinson, F.L. \& Parker, R.B. (1972) The passage of tritiated bacterial endotoxin across intact gingival crevicular epithelium. Journal of Periodontology, 43, 270-276.

Scully, B.E. (1988) Metronidazole. Medical Clinics of North America, 72, 613-621.

Seymour, R.A., Heasman, P.A. (1995) Tetracyclines in the management of periodontal diseases. Journal of Clinical Periodontitis, 22, 22-35.

Seymour, R.A., Heasman, P.A. (1995) Pharmacological control of periodontal disease. II. Antimicrobial agents. Journal of Dentistry, 23, 5-14.

Shenker, B.J. (1987) Immunologic dysfunction in the pathogenesis of periodontal diseases. Journal of Clinical Periodontology, 14, 489-498.

Shinn, D.L.S. (1962) Metronidazole in acute ulcerative gingivitis (letter to the editor). Lancet, 1, 1191.

Silness, J., \& Löe, H. (1964) Periodontal disease in pregnancy. II. correlation between oral hygine and periodontal condition. Acta Odontologica Scandinavica, 22, 121-135.

Slots, J., Mashimo, P., Levine, M.J.\& Genco, R.J.

Periodontal therapy in humans. I. Microbiological and clinical effects of a single course of periodontal scaling and root planing, and of adjuctive tetracycline therapy. Journal of Periodontology, 50, 495-509.

Slots, J. \& Listgarten, M.A. (1988) Bacteroides gingivalis, Bacteroides intermedius and Actinobacillus actinomycetemcomitans in human periodontal diseases. Journal of Clinical Periodontology, 15, 85-93. 
Slots, J. \& Rams, T.E. (1990) Antibiotics in periodontal therapy: advantages and disadvantages. Journal of Clinical Periodontology, 17, 479-493.

Smith, Q.T. \& Geegan, S.J. (1991) Repeated measurement of crevicular fluid parameters at different sites. Journal of Clinical Periodontology 18, 171-176.

Socransky, S.S. (1977) Microbiology and periodontal disease-present status and future considerations. Journal of Periodontology, 48, 497-504.

Socransky, S.S., Haffajee, A.D., Goodson, J.M. \& Lindhe, J. (1984) New concepts of destructive periodontal disease. Journal of Clinical periodontology, 11, $21-32$.

Socransky, S.S. \& Haffajee, A.D. (1991) Microbial mechanisms in the pathogenesis of destructive periodontal disease: a critical assessment. Journal of Periodontal Research, 26, 195-212.

Soder, P.O., Frithiof, L., Wikner, S., Wouters, F., Engstrom, P.E., Rubin, B., Nedlich, U., Soder, B. (1990) The effect of systemic metronidazole after non-surgical treatment in moderate and advance Periodontitis in young adults. Journal of Periodontology, 61, 281-285.

Somerman, M.J., Foster, R.A., Vosteg, G., Progebin, K. \& wynn, R.L. (1988) Effects of minocycline on fibroblast attachment and spreading. Journal of periodontal Research, 23, 154-159.

Sorsa, T., Uitto, V.J., Suomalainen, K., Turto, H. \& Lindy, S. (1987) Human leukocyte collagenase: recent biochemical findings. Proceeding of the Finnish Dental Society, 83, 111-117.

Sorsa T., Uitto V.J., Suomalainen K., Vauhkonen M., Lindy, S. (1988) Comparison of interstitial collagenase from human gingiva, sulcular fluid and polymorphonuclear leukocytes. Journal of Periodontal Research, 23, 386-393. 
Sorsa, T., Ingman, T., Suomalainen, K., Haapasalo, M., Konttinen, Y.T., Lindy, O., Saari, H. \& Uitto, V.-J. (1992) Identification of proteases from periodontopathogenic bacteria as activators of latent human neutrophil and fibroblast-type interstitial collagenases. Infection and Immunity, $60(11), 4491-4495$.

Sorsa, T., Lindy, O., Konttinen, T.Y., Suomalainen, K., Ingman, T., Saari, H., Halinen, H., Lee, H.M., Golub, L. M., Hall, J., \& Simon, S. (1993) Doxacycline in the production of serum alpha-1antitrypsin from human meutrophil collagenase and gelatinase. Antimicrobial Agents and Chemotherapy. $37,592-594$.

Sorsa, T., Ding, Y., Salo, T., Lauhio, A., Teronen, O., Ingman, T., Ohthani, H., Andoh, N., Takeha, S. \& Konttinen, Y.T. (1994) Effects of tetracyclines in neutrophil, gingival, and salivary collagenases: A function and Western-Blot assessment with special reference to their cellular sources in periodontal diseases. Annals of the NewYork Academy of Sciences, 732, 112-131.

Sorsa, T., Ding, Y-L, Ingman, T., Salo, T., Westerlund, U., Haapasalo, M., Tschesche, H., Konttinen, Y.T. (1995) Cellular source, activation and inhibition of dental plaque collagenase. Journal of Clinical Periodontology, 22, 709-717.

Stetler-Stevenson, W. G., Krutzsch, H. C. \& Liotta, L. A. (1989) Tissue inhibitor of metalloproteinase (TIMP2): A new member of the metalloproteinase inhibitor family. Journal of Biological Chemistry, 264, $17374-17378$.

Stricklin, G.P. \& Hoidal, J.R. (1992) In Matrix Metalloproteinases and inhibitors. Birkedal-Hansen, H., Werb, Z., Welgus, H., and Van Wart, Eds. 324 . Gustav Fisher Verlag. New York.

Sueda, T., Bang, J., Cimasoni, G. (1969) Collection of gingival fluid for quantitative analysis. Journal of Dental Research. 48, 159. 
Suomalainen, K., Sorsa, T., Golub, L.M., Ramamurthy, N. Lee, H-M., Uitto, V-J., Saari, H., and Konttinen, Y. T. (1992) Specificity of the anticollagenase action of tetracyclines: Relevance to their antiinflammatory potential. Antimicrobial Agents and Chemotherapy, 36, 227-229.

Sutter, V.L., Jones, M.L., Ghoneim, A.T.M. Antimicrobial susceptibilities of bacteria associated with periodontal disease. Antimicrobial Agents and Chemotherapy, 23, 483-486.

Takamori, K. (1963) The growth stimulating factors for lactobacillus appeared in tissue fluid from gingival crevices. Bulletin of the Tokyo Medical and Dental University. 10, 533-541.

Tally, F.P., Sutter, V.L., Finegold, S.M.

Metronidazole versus anaerobes: in vitro data and initial clinical observations. California Medicine, $117,22-26$.

Teng, Y.T., Sodek, J., McCulloch, C.A.G. (1992) Gingival crevicular fluid gelatinase and its relationship to periodontal disease in human subjects. Journal of Periodontal Research, 27, 544-552.

Tijssen, P. (1985) Practice and theory of enzyme immunoassays. Eds Burdon, R. H. \& Van Knippenberg, P.H. Amsterdam: Elssevier Science Publishers.

Toth, A., Beck, F.M., Beck, E.X., Flaxman, N. \& Rosen, S. (1986) Effect of antimicrobial agents on root surface caries, alveolar bone loss, and microflora in rice rats. Journal of Dental Research, 65, 695697 .

Travis, J. \& Salvesen, G.S. (1983) Human plasma proteinase inhibitors. Annual Review of Biochemistry, 52, 655-709.

Uitto, V.J., Suomalainen, K. \& Sorsa, T. (1990) Salivary collagenase: Origin, characteristic and Relationship to periodontal health. Journal of Periodontal Research, 25, 135-142. 
Uitto, V.J., Firth, J.D., Pan, Y.M., Nip, L., \& Golub, L. M. (1994) Doxacycline and chemically modified tetracyclines inhibit gelatinase A (MMP-2) gen expression in human skin keratinocytes. Annals of the NewYork Academy of Sciences, 732, 140-151.

Van steenberghe, D., Bercy, P. Kohl, J. De Boever, J., Adriaens, P., Vanderfaeillie, A., Adriaenssen, C., Rompen, E., De Vree, H. \& Mccarthy, E.F. (1993) Subgingival minocycline hydrochloride ointment in moderate to severe chronic adult periodontitis: a randomised, double-blind, vehicle-controlled, multicentre study. Journal of Periodontology, 64, 637-644.

Van Winkelhoff, A.J., Rodenburg J.P., Goene R.J., Abbas F, Winkel EG, de-Graaff j. (1989) Metronidazole plus amoxicillin in the treatment of Actinobacillus actinomycetemcomitans associated periodontitis. Journal of Clinical Periodontology, 16, 128-131.

Van Winkelhoff, A.J. \& de Graaff, J. (1991) Microbiology in the management of destructive periodontal disease. Journal of Clinical Periodontology, 18, $406-410$.

Van Winkelhoff, A., Tijhof, C.J., de Graaff, J. Microbiological and clinical results of metronidazole plus amoxicillin therapy in Actinobacillus actinomycetemcomitans-associated periodontitis. Journal of Periodontology, 63, 5257.

Vanooteghem, R., Hutchens, L.H., Bowers, G., Kramer, G., Schallhorn, R., Kinger, R., Crigger, M., Egelberg, J. (1990) Subjective criteria and probing attachment loss to evaluate the effects of plaque control and root debridement. Journal of Clinical Periodontology, 17, 580-587.

Vernillo, A., Ramamurthy N., Lee H-M., Mallya S., Ausmann J., Golub L.M.\& Rifkin, B. (1993) Ros and UMP osteoblast gelatinases: Tetracycline inhibition [Abstract]. Journal of Dental Research, 73, 367. 
Villela, B., Cogen, R.B., Bartolucci, A.A., \& BirkedalHansen, H. (1987) Crevicular fluid collagenase activity in healthy, gingivitis, chronic adult periodontitis and localised juvenile periodontitis patients. Journal of Periodontal Research, 22, 209-211.

Wade, W.G., Addy, M. (1987) Comparison of in vitro activity of niridazole, metronidazole and tetracycline against subgingival bacteria in chronic periodontitis. Journal of Applied Bacteriology, 63, 455-457.

Walker, C.B., Pappas, J.D., Tyler, K.Z., Cohen, S., Gordon, J.M. (1985) Antibiotic susceptibilities of periodontal bacterial: In vitro susceptibilities to eight antimicrobial agents. Journal of Periodontology, 56 (Supp), 67-74.

Walker, C. \& Gordon, J. (1990) The effect of clindamycin on the microbiota associated with refractory periodontitis. Journal of Periodontology, 61, 692698 .

Walsh, M.M., Buchanan, S.A., Hoover, C.I.,Newbrun, E, Taggart E.J, Armitage G.C, Robertson P.B. (1986) Clinical and microbiologic effects of single-dose metronidazole or scaling and root planing in treatment of adult periodontitis. Journal of clinical Periodontology, 13, 151-157.

Welgus, H.G. \& Stricklin, G.P. (1983) Human skin fibroblast collagenase inhibitor. Comparative studies in human connective tissues, serum and amniotic fluid. Journal of Biological Chemistry, 258, 12259-12264.

Wennström, J.L. (1988) What is a clinically healthy periodontium? In: Periodontology today, Int., Congr., Zürich, pp 1-5, Basel: Karger.

Werb, Z. \& Reynolds, J.J. (1974) Stimulation by endocytosis of secretion of collagenase and neutral proteinase from rabbit synovial fibroblasts. Journal of the Experimental Medicine, 140, 14821497. 
Williams, R. C. (1990) Periodontal disease. New England Journal of Medicine. 322, 373-382.

Wilson, M., O'Connor, B. \& Newman, H.N. (1990) Effect of minocycline on subgingival plaque bacteria. Journal of Applied Bacteriology, 69, 228-234.

Wilson, M., O'Connor, B. \& Newman, H.N. (1991) Isolation and identification of bacteria from subgingival plaque with low susceptibility to minocycline. Journal of Antimicrobial chemotherapy, 28, 71-78.

Wilton, J.M.A., Griffiths, G.S., Curtis, M.A., Maiden Gillett, I.R., Wilson, D.T., Sterne, J.A.C. \& Johnson, N.W. (1988) Detection of high-risk groups and individuals for periodontal diseases. Systemic predisposition and markers of general health. Journal of Clinical Periodontology, 15, 339-346.

Woessner, J.F., Jr. (1991) Matrix metalloproteinases and their inhibitors in connective tissue remodelling. Federation of American Societies for Experimental Biology Journal, 5, 2145-2154.

Woessner, J.F., J.R. (1994) The family of Matrix Metalloproteinases. Annals of the NewYork Academy of Sciences, 732, 11-21.

Woolley, D.E. \& Davies, R.M. (1981) Imuunolocalization of collagenase in periodontal disease. Journal of Periodontal Research, 16, 292-297.

Wrana, J.L., Sodek, J.,Ber, R.I., \& Bellows, C.G. (1986) The effects of platelet derived transforming growth factor- $\beta$ on normal human diploid gingival fibroblasts. European Journal of Biochemistry, $159,69-76$.

Yanagimura, M., Koike, F., Hara, K. (1989) Collagenase activity in gingival crevicular fluid and inhibition by tetracyclines. Journal of Dental Research, 68 (Spec Iss), 1691-1693.

Yeung, F.I.S., Newman, H.N., \& Addy, M. (1983) Subgingival metronidazole in acrylic resin vs. chlorhexidine irrigation in the control of chronic periodontitis. Journal of periodontology, 54, 651-657. 
Zucker, S., Wieman, J., Lysik, R,M., Imhof, B., Nagase, H., Ramamurthy, N.S., Liotta, L.A. \& Golub, L.M. (1989) Gelatin-degrading type IV collagenase isolated from human small cell lung cancer, Invasion. Metastasis, 9, 167-181. 


\section{LIST OF PUBLICATION}

The following publication includes material presented as part of this thesis:

Pourtaghi, N., Radvar, M., Moony, J. \& Kinane, D.F. (1995) The effect of subgingival antimicrobial therapy on the levels of stromelysin and tissue inhibitor of metalloproteinases in gingival crevicular fluid. (Submitted) 


\section{The effect of subgingival antimicrobial therapy on the levels of stromelysin and tissue inhibitor of metalloproteinases in gingival crevicular fluid}

N. POURTAGHI, M. RADVAR, J. MOONEY \& D.F. KINANE

Unit of Periodontology, Department of Adult Dental Care, Glasgow Dental Hospital and School, Glasgow, U.K.

Running title: metalloproteinases and local antibiotic therapy

Keywords: Locally delivered; Tetracycline; Minocycline; Metronidazole; stromelysin; tissue inhibitor of metalloproteinases, Periodontitis.

Correspondence:

Professor D.F. Kinane, Unit of Periodontology,

Department of Adult Dental Care, Glasgow Dental Hospital and School, 378 Sauchiehall Street, Glasgow G2 3JZ, Scotland. U.K. 


\section{Abstract}

Recent investigations imply that a key mechanism in the pathogenesis of periodontal disease may be the ability of oral micro-organisms to induce production and /or activation of matrix metalloproteinases (MMPs) in the host tissues. It has been suggested that the pharmacologic inhibition of MMP activity could play an important role in achieving a desirable outcome in periodontal therapy. The efficacy of locally delivered antibiotics on the level of gingival crevicular fluid (GCF) stromelysin (SL) and tissue inhibitor of metalloproteinases (TIMP) on sites with a history of a poor response to mechanical treatment was studied. 52 patients with 4 periodontal pockets $\geq 5$ $\mathrm{mm}$ and bleeding on probing were randomised into four groups of 13 patients. One group received scaling and root planing alone and the other three groups received scaling and root planing plus a locally delivered antimicrobial system. These included $25 \%$ tetracycline fibre, $2 \%$ minocycline gel, and $25 \%$ metronidazole gel. The GCF samples taken at baseline and 6 weeks after treatments were analysed using an enzyme linked immunosorbent assay (ELISA). All treatments resulted in significant improvement in clinical parameters. The pocket depth reduction was significantly greater in the scaling plus tetracycline fibre group than the scaling alone group ( $p=0.003)$. GCF SL levels significantly decreased after adjunctive tetracycline fibre (paired $t$-test, $p=0.020$ ) and minocycline gel (paired t-test, $p=0.023$ ) treatments whereas it remained almost unchanged in the other two groups. While the GCF TIMP level did not change significantly in the scaling and root planing alone group, it significantly increased for all three adjunctive antimicrobial treatments (for tetracycline fibre $p<0.001$, minocycline gel $p=0.005$, metronidazole gel $p$ <0.001). The use of adjunctive locally delivered antimicrobial systems, particularly, the tetracycline family, may offer an advantage in changing the 
metalloproteinase profile of the GCF to a one more compatible with periodontal health.

\section{INTRODUCTION}

Recent investigations imply that a key mechanism in the pathogenesis of periodontal disease may be the ability of oral micro-organisms to induce production and /or activation of matrix metalloproteinases in the host tissues. ${ }^{1-3}$ Stromelysin (SL) is a member of the matrix metalloproteinase family which may play a role in chronic inflammatory periodontitis. ${ }^{4}$ Extracellular control of these enzymes is performed by tissue inhibitor of metalloproteinases (TIMP) ${ }^{5}$ and during normal tissue turnover, inflammation and healing, levels of MMPs and TIMP will change. ${ }^{6}$ The effect of treatment on the levels of MMPs and inhibitors has been to decrease collagenase activity, ${ }^{7-9}$ to decrease the amount of collagenase and stromelysin (SL) produced, and to increase the level of TIMP. ${ }^{10-11}$

It has been suggested that the pharmacologic inhibition of MMP activity could play an important role in achieving a desirable outcome in periodontal therapy. ${ }^{12-16}$ In addition to antimicrobial effects, tetracyclines have been shown to be able to inhibit metalloproteinases (MMPs). However, recent results indicate that the inhibition of MMPs by systemic tetracycline depends on the drug concentration and type ${ }^{2}$ as well as the origin of the MMP. ${ }^{13}$ Therefore, theoretically a great inhibitory effect on MMPs would be expected if higher concentrations of drug could be delivered to the site of action. Recently, there has been increasing interest in the use of locally delivered antibiotics in order to obtain a high concentration of the drug at the target site and to minimise potential systemic adverse effects. We therefore sought to investigate the effects of locally delivered antibiotics on the amount of fibroblast derived SL and TIMP in GCF of sites with advanced periodontal breakdown. 


\section{Materials and Methods}

\section{Selection of subjects and test sites:}

52 subjects with adult periodontitis ( $62 \%$ Female; mean age 45 ; range $30-67$ ) who had previously been treated with subgingival scaling and root planing, were recruited from patients in the Periodontology Unit of Glasgow Dental Hospital and School. All study subjects signed an informed consent form and a full medical history was recorded. Inclusion criteria required that subjects had no history of systemic disease which could influence the course of periodontal treatment, and that they were not on any medications such as antibiotic therapy, which could influence the manifestations of periodontal disease, within the previous 6 months.

Patients selected to take part in the study had at least four periodontal pockets equal to or deeper than $5 \mathrm{~mm}$ with bleeding on probing. The patients were randomly allocated into four groups of 13 patients each, three of which received scaling followed by a locally delivered antibiotic. A total of 208 sites with recurrent periodontitis were examined and treated. Two diseased sites were excluded for technical reasons, one site lost its tetracycline fibre before the 10 day placement period finished and in the scaling group a further site could not be measured. In order to increase the accuracy of the measurements taken and to simplify the harvesting of GCF, the buccal, mesial and anterior sites were preferred to the lingual, distal and posterior sites.

\section{Study Visits}

Site selection was carried out at the screening visit after a full periodontal examination using a PCP-12 probe (Ash, Densply, UK). If the patient fulfilled the criteria, an impression was taken, and an acrylic stent fabricated for attachment level measurements using the Florida probe (Florida Probe Corporation, Florida, USA). 
Oral hygiene instruction was given according to individual needs, but no periodontal instrumentation was carried out at the screening visit. 10 days after the screening visit baseline measurements were taken. The plaque index $(\mathrm{PI})$ of Silness \& Löe (1964) ${ }^{17}$ was recorded to assess the patient's oral hygiene, the severity of gingival inflammation was measured using the gingival index of Lobene et al. (1986). ${ }^{18}$ GCF sampling, volume measurements, bleeding on probing (BOP) and suppuration were registered. Duplicate measurements of pocket depth and attachment level with an acrylic stent were carried out, using the electronic pressure sensitive Florida probe, with a constant force of 20 grams.

At the treatment visits each group received one of the following treatments:

1) Subgingival scaling and root planing under local anaesthesia and the application of tetracycline impregnated fibre (Actisite ${ }^{\circledR}, A L Z A$, corporation, Palo Alto, CA), into the pocket. The fibre was wrapped loosely around the teeth by passing it through the contact points using dental floss or a plastic instrument. The pocket was occupied by fibre without creating excessive tension in the soft tissues. Cyanoacrylate adhesive was applied to the site and a dressing (CoePak, GC America INC. Chicago, IL) was placed. The fibre was removed 10 days later.

2) Subgingival scaling and root planing plus the application of minocycline dental gel (Dentomycin ${ }^{\circledR}$, Lederle Dental Division, Gosport, Hampshire, England), to the pocket until it was overfilled. The excess was removed using cotton wool pellets. This application was repeated 14 days and 28 days after the initial application.

3) Subgingival scaling and root planing plus the application of metronidazole dental gel (Elyzol ${ }^{\circledR}$, Dumex, Denmark), to the pocket until it was overfilled. The excess was removed using cotton wool pellets. The application was repeated 7 days later. 
4) The control group received subgingival scaling and root planing alone.

42 days post treatment all the baseline measurements were repeated for each patient.

During this study other sites received conventional periodontal maintenance care.

\section{Sampling method}

The site to be sampled was isolated with cotton wool rolls and supragingival plaque was carefully removed. The region was dried with a gentle air stream and 30 s later GCF was collected. A Whatman grade 4 paper strip $(2 \times 13 \mathrm{~mm})$ was inserted into the crevice until mild resistance was felt and left in situ for 30s. ${ }^{19}$ The sampling method is reliable and causes no significant disturbances of the gingival blood vessels ${ }^{20}$. The fluid volume on the strip was measured immediately after sampling with a Periotron 6000 (Harco Electronic, Winnipeg, Canada). The jaws of the Periotron were wiped with pure ethanol and then dried between readings. Strips were placed in individual sterile microcentrifuge tubes and stored at $-30^{\circ} \mathrm{C}$ until further processing was carried out. Strips were eluted into $500 \mu$ l of incubation buffer at room temperature using a rotary mixer. The strips were then discarded and the eluate aliquoted and stored at $-30^{\circ} \mathrm{C}$. These aliquots were subsequently analysed for the quantification of SL and TIMP.

\section{Calibration of the Periotron 6000}

In order to transform the Periotron digital readings for each paper strip into volumes, and also to verify the accuracy of the instrument, the following procedure was carried out. Known volumes of PBS plus serum (in equivalent volume) were delivered to Whatman grade 4 paper strip with a Hamilton microsyringe at a range of volumes $(0.05-1 \mu \mathrm{l})$ then Periotron readings taken. 
Each measurement was performed 3 times. The mean value for each volume was used in a linear regression analysis, from which the slope and intercept were used to determine the volumes of GCF collected .

\section{GCF Analysis}

All constituents were assayed by means of sandwich enzyme linked immunosorbent assays (ELISA), based on a modification of the method of Cooksley et al. (1991). ${ }^{21}$ The first antibody, Mac 78 anti-stromelysin for SL and Mac 19 anti-TIMP was coated onto a 96-well polystyrene microplate (Immulon 4, Dynatech Laboratories, Billinghurst, Sussex, U.K). The experimental sample was then added and any antigen present was captured by the immobilised antibody. The plate was then incubated with the second antibody. Biotinylated Mac 15 antibody and rabbit polyclonal antibody were used to reveal TIMP and SL, respectively followed by, in the latter case horseradish peroxide (HRP)-conjugated anti-rabbit IgG (donkey) (Jackson). The polyclonal and monoclonal antibodies were against both proenzyme, and active forms of the enzymes under investigation. The ELISA for TIMP can only detect free TIMP. ${ }^{21}$ All the antibodies were donated by Cell Tech. Ltd. Slough, UK. Finally the plate was incubated with Extravidin-Peroxidase (Sigma). Visualisation was achieved by incubation with tetramethylbenzidine (TMB) (KPL-Dynatech) substrate, and the reaction stopped with $2.5 \% \mathrm{NaF}$. The plate was read at $630 \mathrm{~nm}$, on a Dynatech MR 5000 plate reader. Serial dilutions of standard were run for each individual plate and their optical density (ODs) used in a linear regression analysis from which the slope and intercept were used to determine the sample concentration of SL and TIMP. Results were expressed as $\mathrm{pg} / 30 \mathrm{~s}$ sample. 


\section{Statistical Analysis}

In order to identify the distribution characteristics of the data, it was inspected using histograms and it was noted that the data exhibited a markedly skewed distribution. Log transformation was successful in producing a normal distribution of data within the different groups. The differences in stromelysin levels of each subject, between before and after treatment, for each treatment group, was subjected to paired t-test. The analysis of covariance (ANCOVA) was used on changes of SL and TIMP with the baseline SL or TIMP levels as continuous covariates. If a significant difference was found between treatments, then the post-hoc comparisons were performed by comparing two groups at a time and the probability threshold for statistical significance was adjusted to 0.02 using Bonferroni correction ${ }^{22}$. The Mann-Whitney $U$ test and Two sample t-test were used to analyse the baseline levels of SL and TIMP respectively between bleeding and non-bleeding sites, and between suppurating and non-suppurating sites. Comparisons of SL and TIMP levels at sites with different MGI were performed using the Kruskal-Wallis test. Data were analysed using Minitab statistical package version 9.2.

\section{RESULTS}

Clinical response following the treatments has been reported in a further manuscript (Radvar et al., in press). Briefly, a significant improvement in pocket depth, attachment level, bleeding on probing (BOP) and the MGI scores occurred in all treatment groups. The tetracycline fibre treatment resulted in a significantly greater change in pocket depth than the scaling alone group $(p=0.003)$ (Table 1). In addition, the scaling plus tetracycline fibre treatment resulted in a greater improvement in the MGI scores than the scaling plus minocycline gel treatment $(p=0.002)$, the scaling plus metronidazole gel treatment $(p=0.007)$, and the scaling alone treatment $(p=0.001)$. No significant 
difference existed in the MGI scores between other groups. There were no significant difference in attachment level changes and BOP changes between treatment groups. The GCF volume was reduced siginificantly only the scaling plus tetracycline fibre groups. There was no significant changes in the GCF volume after other treatments.

The mean GCF level of SL decreased in all antibiotic treated groups and this decrease was significant in the tetracycline fibre group (paired t-test, $p=0.020$ ) and the minocycline gel group (paired $t$-test, $p=0.023$ ) (Table 1). Paired $t$-test demonstrated that the level of GCF TIMP significantly increased in all antibiotic treated groups, whereas it decreased in the scaling groups, although not significantly $(p=0.49)$ (Table 1). The analysis of covariance (ANCOVA) on the baseline SL was used to reduce the error resulting from the variation in the baseline. The level of SL at baseline had a significant effect on the SL change following treatment $(p<0.001)$, and this effect was positive i.e. the higher the baseline SL, the higher the reduction of SL. Treatment also had a significant effect on the reduction of $S L(p=0.001)$. Follow-up analysis showed significant differences between; i) tetracycline fibre and scaling alone groups $(p=0.004)$; and ii) minocycline gel and scaling alone groups $(p=0.002)$ (Table 2).

There was a significant difference in TIMP levels between treatment groups $(p=0.007)$. Furthermore, the analysis of TIMP levels revealed that a significant difference existed between the minocycline gel and the scaling alone group $(p=0.018)$, and also between the metronidazole gel and scaling alone group $(p=0.001)$ (Table 3$)$.

The level of SL significantly increased with increasing gingival inflammation $(p=0.028)$. No such relationship was observed between gingival inflammation and GCF TIMP ( $p=0.395)$ (Table 4). The level of SL was higher in sites with bleeding on probing (Mean=20.59 pg/ $/ \mathrm{l}$ ), than sites with no bleeding on probing 


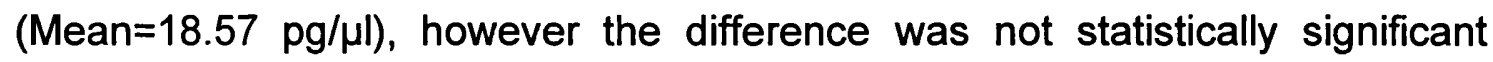
(Mann-Whitney $U$ test, $p=0.47$ ). TIMP levels were lower in bleeding sites

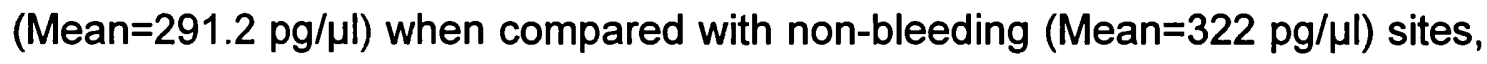
however this was not statistically significant $(p=0.395)$. The level of $S L$ was lower in sites with suppuration (Mean=15.69 pg/ $\mu \mathrm{l}$ ), than sites without suppuration (Mean=21.09 pg/ $\mu \mathrm{l}$ ) but the difference was not significant (MannWhitney $U$ test, $p=0.36$ ). The TIMP level was higher in suppurating sites (Mean=381.9 pg/ $/$ l) compared with the sites which did not show suppuration (Mean=281.1 pg/ $/ \mathrm{ll})$, but this difference was not significant $(p=0.12)$.

\section{DISCUSSION}

This paper reports the effects of the local delivery of antibiotics, as an adjunct to periodontal treatment, on GCF levels of SL, and TIMP, in patients who had previously received periodontal treatment. The clinical results of this investigation have been reported by Radvar et al. (1995). ${ }^{23}$

Golub et al. (1987) ${ }^{24}$ demonstrated that tetracyclines inhibit MMP activity by a mechanism independent of their antimicrobial activity. It was shown that the systemically administrated tetracycline could inhibit collagenases derived from inflammatory sources but inhibition of fibroblast collagenase activity needed higher concentrations than could be achieved by this method of delivery. ${ }^{24-25,12-}$ 13 Therefore, it is hypothesised that locally delivered tetracyclines, due to their high local concentrations can inhibit collagenase from both origins. Metronidazole appears to reduce the prevalence of Porphyromanas gingivalis and spirochetes at active sites in recurrent periodontitis subjects ${ }^{26}$. In addition, the results of Teng et al. (1992) ${ }^{27}$ suggest that metronidazole treatment can cause the reduction of both active and latent forms of gelatinase. The reduction of gelatinase activity is best explained by the antimicrobial effect of 
metronidazole which appears to blunt destructive host immune and inflammatory responses.

It has been suggested that SL may act as a marker of stromal cell involvement in the process of tissue degradation. ${ }^{6}$ Our observation that the mean level of SL decreased after antibiotic treatment could be explained by the fact that local delivery antibiotics plus scaling and root planing may change the microbial flora. This may cause a larger decrease of SL than scaling and root planing alone. However, the greatest decrease was observed in the sites treated by tetracycline or minocycline delivery systems.

Previous studies have also shown that tetracyclines, in addition to decreasing the level of periodontal bacteria, resulting in decrease of MMP activity in the gingiva, could decrease the synthesis of pro-MMPs ${ }^{28-29}$ and also protect the endogenous MMP inhibitors (TIMP-1) and other proteinase inhibitors ( $\alpha 1$ antitrypsin) ${ }^{30}$ from degradation and inactivation by direct and indirect mechanisms. However, it cannot be established from this study whether the effect of the tetracyclines on the level of SL is direct, or indirect through the reduction in microbial challenge which could reduce the microbial proteases and inflammatory mediators such as cytokines, both of which could reduce the levels of human MMP.

After treatment the level of free TIMP increased. This might be due to a reduction in MMPs which would bind to free TIMP. ${ }^{31-33}$ The elevation of GCF TIMP was higher in the metronidazole group than in the other groups. This indicates that the regulation of TIMP is not solely dependent on the MMPs. The level of GCF TIMP was significantly elevated in the patients who were treated with antimicrobial agents. However it did not significantly change in the scaling group. The observation of a greater clinical improvement in all three adjunctive antimicrobial treatment groups compared with the scaling and root planing alone supports our biochemical findings and suggests that the extent of 
healing in the patients who received local delivery antibiotics was greater than the group who only received scaling and root planing.

It has been suggested that MMP and TIMP may be regulated independently. ${ }^{34}$ In conclusion, adjunctive treatment of locally delivered antibiotics, particularly, tetracycline fibre and minocycline gel, is able to decrease the production of SL in the GCF, although whether this is through direct or indirected mechanisms remains unclear. However the correlation between reduced $S L$ and improved clinical parameters is confirmatory (Harian et al. 1995) that SL is a marker of periodontal health. 


\section{References}

1. Birkedal-Hansen $\mathrm{H}$. Role of Matrix Metalloproteinases in Human Periodontal Diseases. Journal of Periodontology, 1993; 64: 474-484.

2. Lee W, Aitken S, Kulkarni G, Birek P, Overall CM, Sodek J, McCulloch CA. Collagenase activity in recurrent periodontitis: relationship to disease progression and doxacycline therapy. Journal of Periodotal Research, 1991; 26: 479-485.

3. Meikle MC, Atkinson SJ, Ward RV, Murphy G, Reynolds JJ. Gingival fibroblasts degrade type I collagen films when stimulated with tumour necrosis factor and interlukin 1: Evidence that breakdown is mediated by metalloproteinases. Journal of periodontal Research, 1989; 24: 207-213.

4. Ingman T, Sorsa T, Michaelis J, Konttinen YT. Matrix metalloproteinases$1,-3,-8$ in adult periodontitis in situ. Annals of the Newyork Academy of Sciences, 1994; 732: 459-461.

5. Murphy G, Koklitis P, Carne AF. Dissociation of tissue inhibitor of Metalloproteinases (TIMP) from enzyme complex yields fully active inhibitor. Biochemical Joumal, 1989; 261: 1031-1034.

6. Birkedal-Hansen $H$, Moore WGI, Bodden MK, Windsor LJ, BirkedalHansen B, Decario A, Engler JA. Matrix Metalloproteinases: A Review. Critical review in oral Biology and Medicine, 1993; 4: 197-250.

7. Larivee L, Sodek J, Ferrier JM. Collagenase and collagenase inhibitor activities in crevicular fluid of patients receiving treatment for localised juvenile periodontitis. Journal periodontal Research, 1986; 21: 702-715.

8. Hakkarainen $\mathrm{K}$, Uitto $\mathrm{V}-\mathrm{J}$, Ainano $\mathrm{J}$. Collagenase activity and protein content of sulcular fluid after scaling and occlusal adjustment of teeth with deep periodontal pockets. Journal of periodontal Research, 1988; 23: 204-210. 
9. Yanagimura M, Koike F, Hara $K$. Collagenase activity in gingival crevicular fluid and inhibition by tetracyclines. Journal of Dental Research, 1989; 68: (Spec Iss), 1691-1693.

10. Haerian A, Adonogianaki E, Mooney J, Manos A, Kinane DF. Effect of treatment on gingival crevicular collagenase, stromelysin and tissue inhibitor of metalloproteinases and their ability to predict response to treatment. Joumal of Clinical Periodontology, 1995; In press.

11. Haerian A, Adonogianaki E, Mooney J, Docherty JP, Kinane DF. Gingival crevicular stromelysin, collagenase and tissue inhibitor of metalloproteinases levels in healthy and diseased sites. Journal of Clinical Periodontology, 1995; 22: 505-509.

12. Golub LM, Wolff M, Lee H-M, McNamara TF, Ramamurthy NS, Zambom $\mathrm{J}$, Ciancio S. Further evidence that tetracyclines inhibit collagenase activity in human crevicular fluid and from other mammalian sources. Journal of Periodontal Research, 1985; 20: 12-23.

13. Golub LM, Ramamurthy NS, McNamara TF, Greenwald RA, Rifkin BR. Tetracyclines inhibit connective tissue breakdown: new therapeutic implications for an old family of drugs. Critical Reviews in Oral Biology of Medicine, 1991; 2: 297-322.

14. Golub LM, Evans RT, McNamara TF, Lee HM, Ramamurthy NS. A non-antimicrobial tetracycline inhibits gingival matrix metalloproteinases and bone loss in prophyromonas gingivalis-induced periodontitis in rats. Annals of the Newyork Academy of Sciences, 1994; 732: 96-111.

15. Sorsa $T$, Ding $Y$, Salo $T$, Lauhio A, Teronen $O$, Ingman $T$, Othani $H$, Andoh N, Takeha S, Konttinen YT. Effects of tetracyclines in neutrophil, gingival, and salivary collagenases: A function and Western-Blot assessment with special reference to their cellular sources in periodontal diseases. Annals of the NewYork Academy of Sciences, 1994; 732: 112-131. 
16. Suomalainen K, Sorsa T, Golub LM, Ramamurthy N, Lee H-M, Uitto V-J, Saari $H$, Konttinen $Y T$. Specificity of the anticollagenase action of tetracyclines: Relevance to their anti-inflammatory potential. Antimicrobial agents and chemotherapy, 1992; 36: 227-229.

17. Silness J, Löe H. Periodontal disease in pregnancy. II. correlation between oral hygine and periodontal condition. Acta Odontologica Scandinavica, 1964; 22: 112-135.

18. Lobene RR, Weatherford T, Ross NM, Lamm RA, Manaker L. A modified gingival index for use in clinical trials. Clinical Preventive Dentistry; 1986; 8: 3-6.

19. Griffith GS, Curtis MA. Wilton JMA. Selection of a filter paper with optimum properties for the collection of gingival crevicular fluid. Journal of periodontal Research, 1988; 23: 33-38.

20. Gustafsson A, Asman B, Bergstrom K, Soder P-O. Granulocyte elastase in gingival crevicular fluid. A possible discriminator between gingivitis and periodontitis. Journal of Clinical Periodontology, 1992; 19: 535-540.

21. Cooksley S, Hipkiss JB, Tickle SP, Holmes-Levers E, Docherty AJ, Murphy G, Lowson AD. Immunoassay for the detection of human collagenase, stromelysin, tissue inhibitor of metalloproteinases (TIMP) and enzyme-inhibitor complex. Matix, 1991; 10: 285-291.

22. Brown RA, Swanson Beck J. A non-algebraic to their appropriate use in biomedical research and pathology practice. 3 Analysis of variance and distribution-free methods. Journal of Pathology, 1988; 41: 1256-1262.

23. Radvar M, Pourtaghi N, Kinane DF. A comparative evaluation of 3 locally delivered antimicrobial systems used in conjunction with scaling and root planing in persistent periodontal pockets. Journal of Periodontology, 1995. submitted for publication. 
24. Golub LM, McNamara TF, D'Angelo GD, Greenwald, Ramamurthy NS. A non-antibacterial chemically-modified tetracycline inhibitors mammalian collagenase activity. Journal of Dental Research, 1987; 66: 1310-1314.

25. Golub LM, Ciancio S, Ramamurthy NS, Leung M, McNamara TF. Effect on gingival and crevicular fluid collagenase activity in humans. Journal of Periodontal Research, 1990; 25: 321-330.

26. Loesche WJ, Syed SA, Morrision EO, Kerry GA, Higgins T, Stoll J. Metronidazole in periodontitis I. clinical and bacteriological result after 15 to 30 weeks. Journal of Periodontology, 1984; 55: 325-335.

27. Teng YT, Sodek J, McCuttoch CAG. Gingival crevicular fluid gelatinase and its relationship to periodontal disease in human subjects. Journal of Periodontal Research, 1992; 27: 544-552.

28. Rifkin BR, Vernillo AT, Golub LM. Blocking periodontal disease progression by inhibiting tissue-destructive enzyme: a potential therapy role for tetracycline and their chemically-modified analysis. Joumal of periodontology, 1993; 64: (suppl.): 819-827.

29. Uitto VJ, Firth JD, Pan YM, Nip L, Golub LM. Doxacycline and chemically modified tetracyclines inhibit gelatinase A (MMP-2) gen expression in human skin keratinocytes. Annals of the NewYork Academy of Sciences, 1994; 732: 140-151.

30. Sorsa $T$, Lindy $O$, Konttinen TY, Suomalinen $K$, Ingman $T$, Saari $H$, Halinen $H$, Lee HM, Golub LM, Hall J, Simon S. Doxacycline in the production of serum alpha-1-antitrypsin from human meutrophil collagenase and gelatinase. Antimicrobial Agents and Chemotherapy, 1993; 37: 592-594.

31. Cawston TE, Murphy G, Mercer E, Calloway WA, Hazieman BL, Reynolds JJ. The interaction of purified rabbit bone collagenase with purified rabbit bone metalloproteinase inhibitions. Biochemical Journal, 1983; 211: 313-318. 
32. Murphy G, Willenbrock F, Crabbe T, O'Shea M, Ward R, Atkinson S, O'Connell J, Docherty A. Regulation of Matrix Metalloproteinase Activity. Annals of the NewYork Academy of Sciences, 1994; 732: 31-41.

33. Howard. E.W., Bullen, E. C., Banda. M. J. Preferential inhibition of 72 and 92-KDa gelatinases by tissue inhibitor of Metalloproteinases.2. Journal of Biological Chemistry, 1991; 266: 13070-13075.

34. Overall CM. Regulation of tissue inhibitor of matrix metalloproteinase expression. Annals of the NewYork Academy of Sciences, 1994; 732: 51-65. 
Table 1. Mean differences (baseline - after treatment) of biochemical and clinical parameters after therapy in four treatment groups.

\begin{tabular}{|c|c|c|c|c|c|c|c|c|c|}
\hline \multirow[t]{2}{*}{ Treatment } & \multicolumn{3}{|c|}{$\Delta \mathbf{S L}$} & \multicolumn{2}{|c|}{$\Delta$ TIMP } & \multicolumn{2}{|c|}{$\begin{array}{l}\text { Attachment } \\
\text { gain }\end{array}$} & \multicolumn{2}{|c|}{$\begin{array}{l}\text { Pocket depth } \\
\text { reduction* }\end{array}$} \\
\hline & $n$ & $\begin{array}{l}\text { mean\# } \\
\text { (SEM) }\end{array}$ & $\mathrm{p}^{\star \star \star}$ & $\begin{array}{l}\text { mean\# } \\
\text { (SEM) }\end{array}$ & $\mathrm{p}^{\star \star}$ & $\begin{array}{l}\text { mean } \\
\text { (SEM) }\end{array}$ & $\mathbf{p}^{\star \star \star}$ & $\begin{array}{l}\text { mean } \\
\text { (SEM) }\end{array}$ & $\mathbf{p}^{\star \star}$ \\
\hline S \&TC fibre & 51 & $\begin{array}{l}0.11 \\
(0.05)\end{array}$ & 0.020 & $\begin{array}{l}-0.32 \\
(0.07)\end{array}$ & $<0.001$ & $\begin{array}{l}0.75 \\
(0.12)\end{array}$ & $<0.001$ & $\begin{array}{l}1.35 \\
(0.17)\end{array}$ & $<0.001$ \\
\hline S \& Min gel & 52 & $\begin{array}{l}0.13 \\
(0.06)\end{array}$ & 0.023 & $\begin{array}{l}-0.22 \\
(0.08)\end{array}$ & 0.005 & $\begin{array}{l}0.40 \\
(0.14)\end{array}$ & 0.013 & $\begin{array}{l}0.813 \\
(0.13)\end{array}$ & $<0.001$ \\
\hline S \& Met gel & 52 & $\begin{array}{l}0.01 \\
(0.30)\end{array}$ & 0.730 & $\begin{array}{l}-0.38 \\
(0.07)\end{array}$ & $<0.001$ & $\begin{array}{l}0.59 \\
(0.14)\end{array}$ & 0.001 & $\begin{array}{l}0.965 \\
(0.18)\end{array}$ & $<0.001$ \\
\hline $\mathbf{s}$ & 51 & $\begin{array}{l}-0.01 \\
(0.06)\end{array}$ & 0.940 & $\begin{array}{l}+0.05 \\
(0.07)\end{array}$ & 0.490 & $\begin{array}{l}0.26 \\
(0.11)\end{array}$ & 0.032 & $\begin{array}{l}0.604 \\
(0.14)\end{array}$ & $<0.001$ \\
\hline
\end{tabular}

* The S \& TC showed a significantly greater pocket depth reduction than the $S(p=0.003)$.

** Paired t-test, baseline and post-therapy for each treatment

$S \& T C$ fibre= Scaling and Tetracycline fibre

$S \&$ Min gel= Scaling and Minocycline gel

$S \&$ Met gel= Scaling and Metronidazole gel

$S=$ Scaling alone

\# log transformed data

Table 2. Statistical analysis of changes of GCF level of Stromelysin between test groups $(n=206)$.

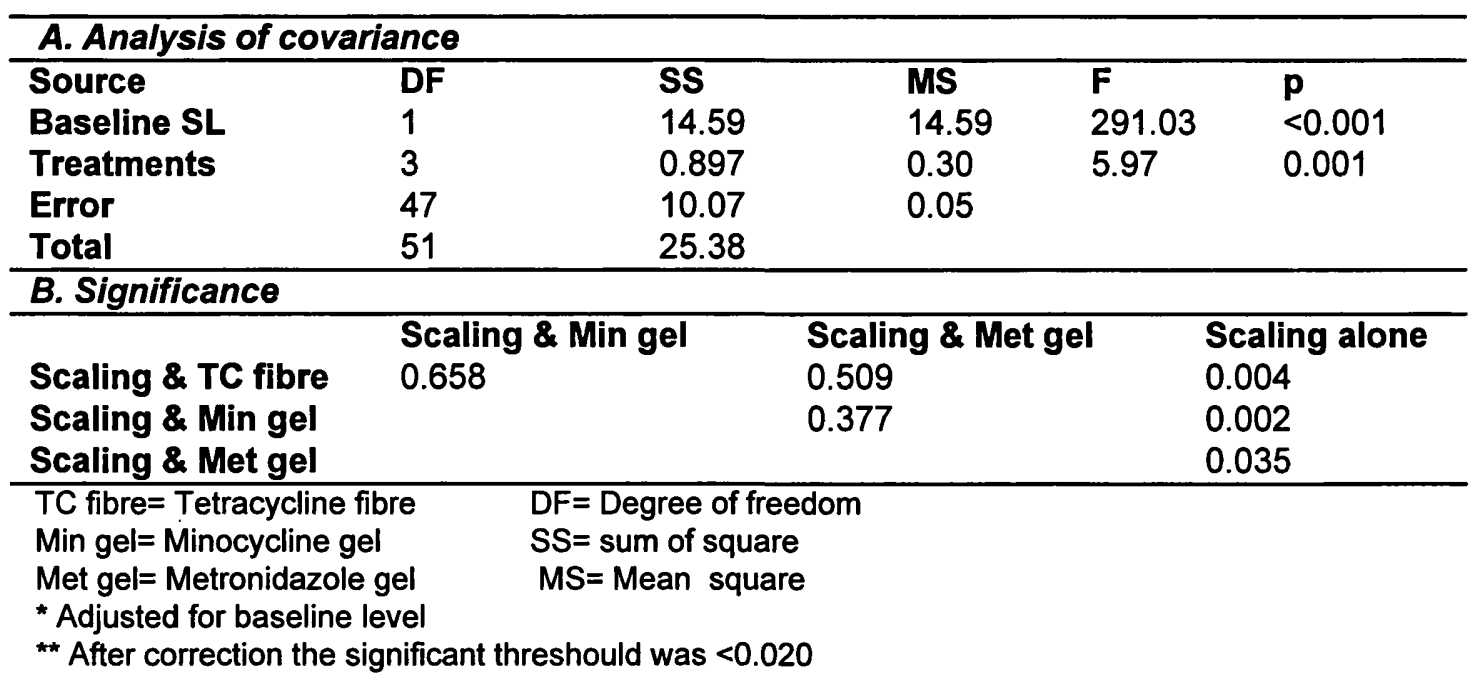


Table 3. Statistical analysis of changes of GCF level of TIMP between test groups $(n=206)$.

\begin{tabular}{|c|c|c|c|c|c|c|}
\hline \multicolumn{7}{|c|}{ A. Analysis of covariance } \\
\hline Source & $\overline{D F}$ & \multicolumn{2}{|c|}{ SS } & MS & $\mathbf{F}$ & \\
\hline Baseline TIMP & 1 & \multicolumn{2}{|c|}{20.53} & 20.53 & 119.25 & $<0.001$ \\
\hline Treatments & 3 & \multicolumn{2}{|c|}{2.13} & 0.71 & 4.13 & 0.007 \\
\hline Error & 201 & \multicolumn{2}{|c|}{34.61} & 0.17 & & \\
\hline Total & 205 & \multicolumn{3}{|c|}{60.73} & & \\
\hline \multicolumn{7}{|l|}{ B. Significance } \\
\hline $\begin{array}{l}\text { Scaling \& TC fibre } \\
\text { Scaling \& Min gel } \\
\text { Scaling \& Met gel }\end{array}$ & \multicolumn{2}{|c|}{$\begin{array}{l}\text { Scaling \& Min gel } \\
0.503\end{array}$} & \multicolumn{2}{|c|}{$\begin{array}{l}\text { Scaling \& Met gel } \\
0.170 \\
0.400\end{array}$} & \multicolumn{2}{|c|}{$\begin{array}{l}\text { Scaling alone } \\
0.036 \\
0.018 \\
0.001\end{array}$} \\
\hline \multicolumn{2}{|c|}{$\begin{array}{l}\text { TC fibre=Tetracycline fibre } \\
\text { Min gel= Minocycline gel } \\
\text { Met gel= Metronidazole gel } \\
{ }^{*} \text { Adjusted for baseline leve }\end{array}$} & \multicolumn{3}{|c|}{$\begin{array}{l}\text { DF= Degree of freedom } \\
\text { SS= Sum of square } \\
M S=\text { Mean square }\end{array}$} & & \\
\hline
\end{tabular}

Table 4. Comparison mean (SEM) of GCF SL and TIMP levels for different gingival inflammation scores at baseline, using Kruskal-wallis test.

\begin{tabular}{l|lll}
\hline & $\mathbf{n}$ & Stromelysin(pg/30s) & TIMP(pg/30s) \\
\hline MGI=0 & 6 & $16.70(4.12)$ & $295.0(115)$ \\
MGI=1 & 29 & $11.50(1.03)$ & $234.0(53.2)$ \\
MGI=2 & 112 & $18.64(2.28)$ & $326.1(38.9)$ \\
MGI=3 & 59 & $28.45(6.53)$ & $263.4(38.1)$ \\
\hline P value & & 0.028 & 0.395 \\
\hline MGI= Modified gingival index & &
\end{tabular}

